\title{
Comprehensive Proteomic Profiling of the Brassica carinata and Triticale Mature Stigma and Pollen
}

\author{
by
}

Reneé Robinson

A thesis submitted to the Faculty of Graduate and Postdoctoral Affairs in partial fulfillment of the requirements for the degree of

Doctor of Philosophy

In

Biology

Department of Biology

Carleton University

Ottawa, Ontario, Canada

(C) 2018

Reneé Robinson 


\section{Abstract}

Plant sexual reproduction commences with the interaction of the mature stigma (the receptive portion of the female reproductive organ) and the mature pollen (the male gametophyte). Proteins present at their mature developmental stages are therefore likely to reflect their upcoming reproductive roles. In this study, gel-free shotgun proteomics was used to investigate the proteomes of the mature stigma and pollen grain in two species that are highly related to Canada's most important crops. We identified 7703 and 5608 Brassica carinata stigma and pollen proteins, and 11533 and 2977 triticale stigma and pollen proteins, respectively. These represent the largest Brassicaceae and Triticeae pollen and stigma proteome datasets to date. In addition, this is the first comparative analysis of developing stigmas, and it was performed using isobaric tag for relative and absolute quantification (iTRAQ). There were $251 \mathrm{~B}$. carinata proteins found to be differentially abundant during stigma maturation with 154 proteins that had increased abundance, while 97 decreased in abundance. For triticale, 647 differentially abundant stigma proteins were identified with 305 and 342 proteins increasing and decreasing in abundance respectively.

A comparison of the $B$. carinata and triticale stigma or pollen proteomes suggested considerable functional conservation between the two species despite their very different morphology. Although the proteomes exhibited similar functional enrichment trends, both species displayed significant differences between their pollen and stigma proteomes, which reflected their functional specialization. Altogether, the results of this study represent an invaluable resource for the further study of pollen and stigma 
development and interactions, and will potentially facilitate our understanding of plant reproduction in other members of the Brassicaceae (e.g. B. napus, B. oleracea, B. rapa) and Triticeae (wheat, barley, rye). 


\section{Preface}

This dissertation follows the integrated thesis format and as such, Chapters 2 and 3 will be submitted for publication in a peer-reviewed journal.

My contributions:

1. In partnership with my supervisor Dr. Laurian Robert, I developed the research question and experimental design.

2. All experiments and data analysis were performed by me with the exception of the online RP nanoscale capillary LC coupled to tandem mass spectrometry (MS/MS), the iTRAQ labeling and iTRAQ 2D HPLC- MS/MS that was outsourced to the Plate-forme protéomique, Centre Génomique de Québec (http://proteomique.crchul.ulaval.ca/en/), the mass BLASTs were performed by Phil Couroux and the light microscopy and scanning electron microscopy was performed by Elizabeth Routly.

3. Drafts of this thesis were prepared and edited by myself under the guidance of Dr. Laurian Robert. 


\section{Acknowledgments}

I would like to thank my supervisor Dr. Laurian Robert for the opportunity to pursue a $\mathrm{PhD}$ under his mentorship. I would especially like to thank him for his patience, guidance and support throughout this journey.

I would also especially like to thank Dr. Jeff Smith for allowing me to attend his lab meetings and helping me to understand some of the proteomics theory that I found challenging in the beginning of my studies. In addition, I would like to thank my cosupervisor Dr. Tim Xing and my thesis advisory committee member, Dr. Doug Johnson for all their helpful suggestions and guidance along the way.

Thank you to the members of my lab, Dr. Mohsin Zaidi for showing me how to do the DAPI stains, Dr. Vishwanath Sollapura and Elizabeth Routly for assisting with the SEM and the light microscopy. Enormous thanks to Phil Couroux for his assistance with the mass BLASTs and showing me how to use Access, and just being there for me whenever I had any computer problems. I am tremendously grateful.

Special thanks to Dr. Shea Miller for allowing me to use her fluorescence microscope and also Dr. Gopal Subramaniam for allowing me to use his HPLC apparatus. I would also like to thank Natalie Labbé for her assistance with HPLC, and Dr. Karla Fox for all her help with understanding some of the statistical elements to my research.

Thank you to my mother Yvonne Robinson for her overwhelming love, support, guidance, prayers and encouragement during my life and during this process. Thank you mom for always being a strong shoulder to lean on. I am and will forever be grateful. Thanks to my step-dad Jay Jackson, my dear friends Dilini Kabetu and Rathiha Egbert 
for their friendship, prayers and support during this process, and my good friend Corei Taylor for his help with some of the literature searches. Special thanks to my best friend Sid Sudiacal who has kept me accountable throughout our 15+ years of friendship and whose support and encouragement during this journey has been incredible.

Lastly, I would like to thank God for opening doors, giving me the strength to persevere, and providing wonderful people in my life to guide, mentor and encourage me along this journey. 


\section{Table of Contents}

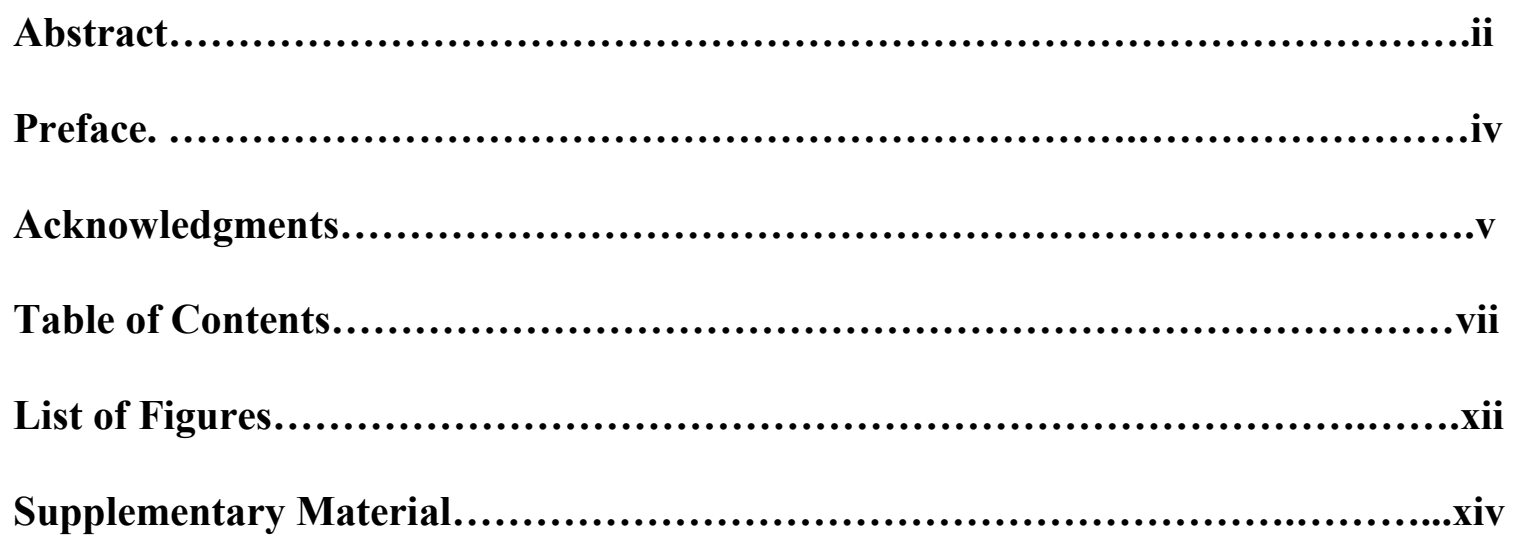

Chapter 1. General Introduction...................................................1

1.1 Brassica carinata ......................................................

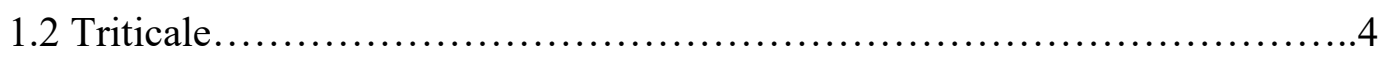

1.3 Plant Reproductive Systems........................................... 6

1.4 Sexual Plant Reproduction...........................................6

1.4.1 Female Reproductive Organs.................................6

1.4.2 Male Reproductive Organs....................................

1.4.3 Pollen Dissemination......................................... 9

1.4.4 Pollen-Stigma Interactions................................... 10

1.5 Proteomics.................................................................. 12

1.5.1 Gel-based Proteomics........................................13

1.5.2 Gel-free Proteomics.......................................... 15

1.5.3 Quantitative Proteomics: Label-based vs. Label-free.................18

1.5.4 Tandem Mass Spectrometry ....................................20 
1.5.5 Orbitrap Fusion ${ }^{\mathrm{TM}}$ Tribrid $^{\mathrm{TM}}$ Mass Spectrometer.................21

1.5.6 Bioinformatics.............................................22

1.6 Previous Studies of the Stigma and Pollen Proteomes....................23

1.7 Hypothesis...................................................... 26

\section{Chapter 2. The Brassica Mature Stigma and Pollen Proteomes: Preparing to}

Meet........................................................................27

2.1 Introduction....................................................27

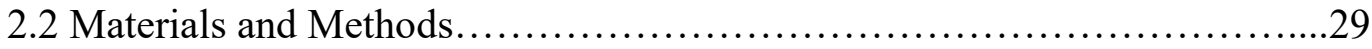

2.2.1 Plant Material.............................................29

2.2.2 Tissue Collection and microscopy...........................29

2.2.3 Trichloroacetic Acid (TCA) Direct Protein Precipitation...........32

2.2.4 iTRAQ Analysis.......................................... 32

2.2.5 Global Mass Spectrometry Analysis............................36

2.2.6 General Data Analysis......................................39

2.2.7 String Network Analysis...................................40

2.3 Results and Discussion...........................................40

2.3.1 Functional Classification of B. carinata Mature Pollen and Stigma

Proteins............................................40

2.3.2 Differential Protein Abundance During B. carinata Stigma

Maturation...............................................49

2.3.3 Pollen- Pistil Interactions.................................51

2.3.3.1 Pollen Adhesion.....................................51 
2.3.3.2 Pollen Recognition...................................54

2.3.3.3 Pollen Hydration........................................55

2.3.3.4 Pollen Germination....................................58

2.3.3.5 Pollen Tube Growth..................................60

2.3.3.6 Pollen Tube Guidance................................70

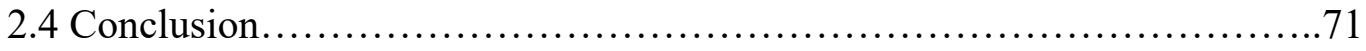

\section{Chapter 3. The Analysis of the Triticale (x Triticosecale Wittmack) Mature Stigma} and Pollen Proteomes...............................................73

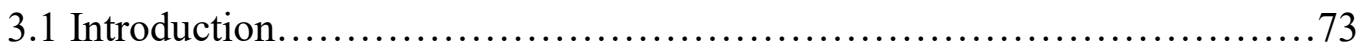

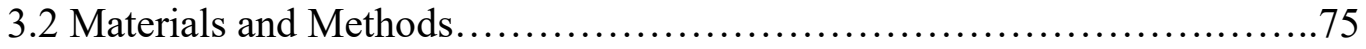

3.2.1 Plant Material.................................................... 75

3.2.2 Tissue Collection and Microscopy ............................. 75

3.2.3 Trichloroacetic Acid (TCA) Direct Protein Precipitation............78

3.2.4 iTRAQ Analysis.......................................... 78

3.2.5 Global Mass Spectrometry Analysis............................82

3.2.6 General Data Analysis........................................... 85

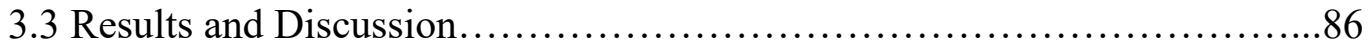

3.3.1 Functional Classification of Triticale Mature Stigma and Pollen

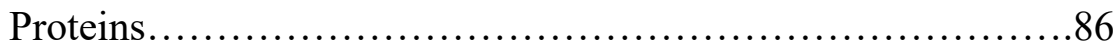

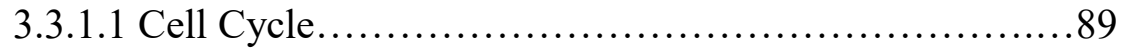

3.3.1.2 Chromosome Organization...........................99

3.3.1.3 Development......................................93 
3.3.1.4 Nucleobase-containing Compound Metabolism, Gene Expression, RNA Metabolism, and Transcription.........99

3.3.1.5 Protein Metabolism.....................................101

3.3.1.6 Response to Stimulus and Defense Response............102

3.3.1.7 Transport......................................... 106

3.3.2 Differential Protein Abundance During Triticale Stigma

Maturation............................................... 107

3.3.2.1 Carbohydrate Metabolic Process.........................109

3.3.2.2 Cell Wall Organization or Biogenesis.................109

3.3.2.3 Response to Stress......................................110

3.3.2.4 Defense..............................................110

3.3.2.5 Signaling ......................................... 111

3.3.2.6 Transport.......................................... 111

3.3.2.7 Cytoskeleton.........................................112

3.3.2.8 Metabolic Process....................................112

3.3.2.9 Protein Metabolic Process and Protein

Phosphorylation........................................113

3.3.2.10 Regulation of Transcription..........................114

3.3.3 Brassica vs. Triticale Comparison...............................114

3.3.3.1 The Global Proteome..................................115

3.3.3.2 Functional Enrichment.................................118

3.3.3.3 Differential Protein Abundance in the Maturing

Stigma ............................................... 122 
3.4 Conclusion..................................................... 125

Chapter 4. General Discussion...................................................127

4.1 Future Directions...........................................133

4.2 General Conclusion.............................................. 140

References........................................................................141 


\section{List of Figures}

Figure 1.1 Schematic diagram of the $B$. carinata flower..........................

Figure 1.2 Schematic diagram of the triticale flower.............................

Figure 2.1 Microscopy of $B$. carinata mature pollen and stigma at the bicellular pollen (BCP) and tricellular pollen (TCP) stages of development........31

Figure 2.2 Comparison of the functional distribution of the B. carinata mature stigma and pollen proteomes .42

Figure 2.3 Comparison of the number of enriched and under-represented functional sub-categories of the $B$. carinata mature stigma and pollen proteomes.

Figure 2.4 Comparison of the number of unique enriched and under-represented functional sub-categories of the $B$. carinata mature stigma and pollen proteomes

Figure 2.5 Percentage of proteins that increased and decreased in abundance during B. carinata stigma maturation. .50

Figure 3.1 Microscopy of mature triticale pollen and stigma at the bicellular pollen (BCP) and tricellular pollen (TCP) stages of development .76

Figure 3.2 Comparison of the functional distribution of the triticale mature stigma and pollen proteomes

Figure 3.3 Comparison of the number of enriched functional sub-categories of the triticale mature stigma and pollen proteomes 
Figure 3.4 Comparison of the number of unique enriched functional sub-categories of the triticale mature stigma and pollen proteomes.

Figure 3.5 Percentage of proteins that increased and decreased in abundance during triticale stigma maturation 108

Figure 3.6 Comparison of the functional distribution of B. carinata and triticale mature stigma proteomes

Figure 3.7 Comparison of the functional distribution of $B$. carinata and triticale mature pollen proteomes

Figure 3.8 Comparison of the enriched and under-represented functional categories of B. carinata and triticale mature stigma proteomes.

Figure 3.9 Comparison of the enriched and under-represented functional categories of B. carinata and triticale mature pollen proteomes 120

Figure 3.10 Comparison of the number of proteins that increased in abundance during stigma maturation for $B$. carinata and triticale.

Figure 3.11 Comparison of the number of proteins that decreased in abundance during stigma maturation for $B$. carinata and triticale.

Figure 4.1 Network of putative $B$. carinata pollen protein-protein interactions involved in pollen tube growth. .135

Figure 4.2 Network of putative $B$. carinata stigma protein-protein interactions involved in pollen hydration.

Figure 4.3 Network of putative B. carinata pollen protein-protein interactions involved in pollen hydration. 


\section{Supplementary Material}

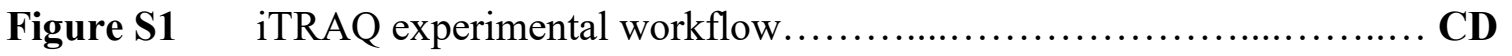

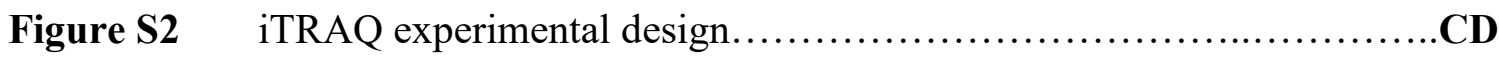

Figure S3 Global experimental workflow.................................... CD

Figure S4 Scanning Electron Microscopy of the B. carinata pollen germinating on the surface of the stigma........................................

Table S2.1 List of all the B. carinata mature stigma and pollen proteins

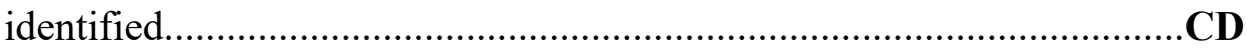

Table S2.2 List of all the $B$. carinata mature stigma proteins divided into functional categories...................................................

Table S2.3 List of all the B. carinata mature pollen proteins divided into functional

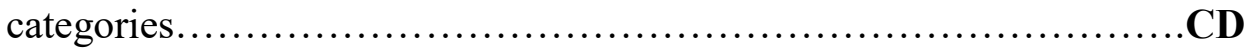

Table S2.4 List of $B$. carinata mature stigma enriched and under-represented functional sub-categories and their constituent proteins...............D

Table S2.5 List of B. carinata mature pollen enriched and under-represented functional sub-categories and their constituent proteins...............D

Table S2.6 List of B. carinata proteins that increased in abundance during stigma

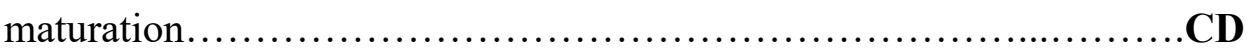

Table S2.7 List of $B$. carinata proteins that decreased in abundance during stigma

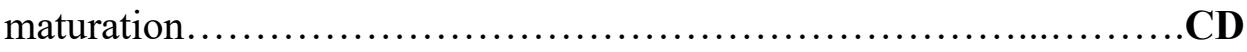

Table S3.1 List of all the triticale mature stigma and pollen proteins identified......CD 
Table S3.2 List of all the triticale mature stigma proteins divided into functional

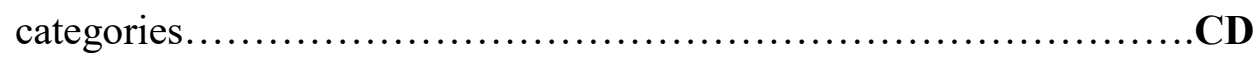

Table S3.3 List of all the triticale mature pollen proteins divided into functional

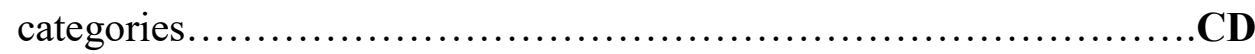

Table S3.4 List of triticale mature stigma enriched and under-represented functional sub-categories and their constituent proteins...............D

Table S3.5 List of triticale mature pollen enriched and under-represented functional sub-categories and their constituent proteins...............

Table S3.6 List of triticale proteins that increased in abundance during stigma

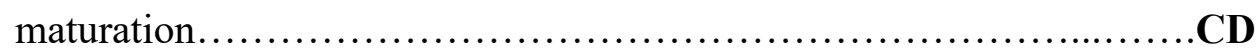

Table S3.7 List of triticale proteins that decreased in abundance during stigma maturation..................................................

Table S4.1 Total number of stigma proteins identified from the global analyses 1, 2

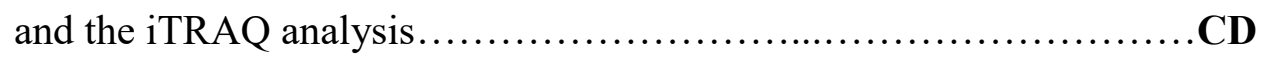

Table S4.2 Summary table of number of spectra, peptides and proteins.............CD 


\section{Chapter 1}

\section{General Introduction}

Flowering plants, formally classified as Magnoliophyta, but informally known as angiosperms, are the largest division within the plant kingdom. Angiosperms are divided into two main classifications; Monocots and Dicots. One of the main differences between the two is their floral structure. Flowers are important for sexual reproduction as they contain the reproductive organs of the plant (Mauseth, 1998). Many monocots and dicots are monoclinous, containing both the female (pistil) and male (stamen) reproductive organs in the same flower (Vines, 1894). Reproduction begins when pollen, released from the stamen, interacts with the stigma of the pistil, and therefore, uncovering molecular factors involved in pollen-stigma interactions is paramount to the understanding of plant reproduction. Since proteins are the mediators of physiological function within an organism, uncovering the proteome (the entire protein complement of a cell, tissue or organism) of the mature stigma and mature pollen grain, as well as the proteins that change in abundance as the stigma matures, should contribute to unraveling the molecular processes involved in pollen-stigma interactions.

In this study, gel-free proteomics was used in order to elucidate key proteins underlying molecular mechanisms regulating pollen and stigma interactions in the monocot triticale and dicot Brassica carinata. As representatives of Canada's most cultivated crops, acquiring a greater understanding of the molecular processes involved in their reproduction will aid in the development of innovative approaches to managing crop reproduction. 


\subsection{Brassica carinata}

Brassica carinata $(2 \mathrm{n}=4 \mathrm{x}=34 ; \mathrm{BBCC})$, otherwise known as Ethiopian mustard, belongs to the Brassiceae tribe, which is a part of the Brassicaceae (Cruciferae) family. It is a product of a natural hybridization between $B$. nigra $(2 \mathrm{n}=2 \mathrm{x}=16 ; \mathrm{BB})$ and $B$.

oleracea $(2 \mathrm{n}=2 \mathrm{x}=18 ; \mathrm{CC})$ (Alemayehu and Becker, 2002; Kristal and Lampe, 2002;

Lysak et. al., 2005; Rakow, 2004). B. carinata is a robust plant having a high tolerance to biotic and abiotic stress. It also has a large seed size and high seed yield, along with high protein and oil content. Because of its high erucic acid and glucosinolate content, $B$. carinata is undesirable for human consumption. However, its agronomical qualities make it suitable for industrial use. For example, due to its high oil content, there is a viable market for the production of B. carinata biofuel (Alexander et. al., 2008; Getinet et. al., 1994; Johnson, 2013).

The floral structure of $B$. carinata is similar to that of other members of its genus. It has four sepals and four petals, and it is monoclinous having its male reproductive parts (four long stamens and two shorter stamens, each consisting of an anther and a filament) and female reproductive part (pistil consisting of the papillate stigma, the style and the ovary) within the same flower. The ovary is bicarpelar having parietal placentation and is separated by a false septum. The flower also contains 4 nectaries in total; two lateral/inner nectaries (between the ovary and the short stamens) and two median/outer nectaries (between the long stamens and the petals) (Figure 1.1) (Mussury and Fernandes, 2000; Kudo, 2003; Davis et. al., 1996). It is believed that the short stamens function to assist in cross-pollination as they act as a barrier against easy access to the nectary for nectar feeders resulting in a longer stay for pollinators (Kudo, 2003). B. carinata is 


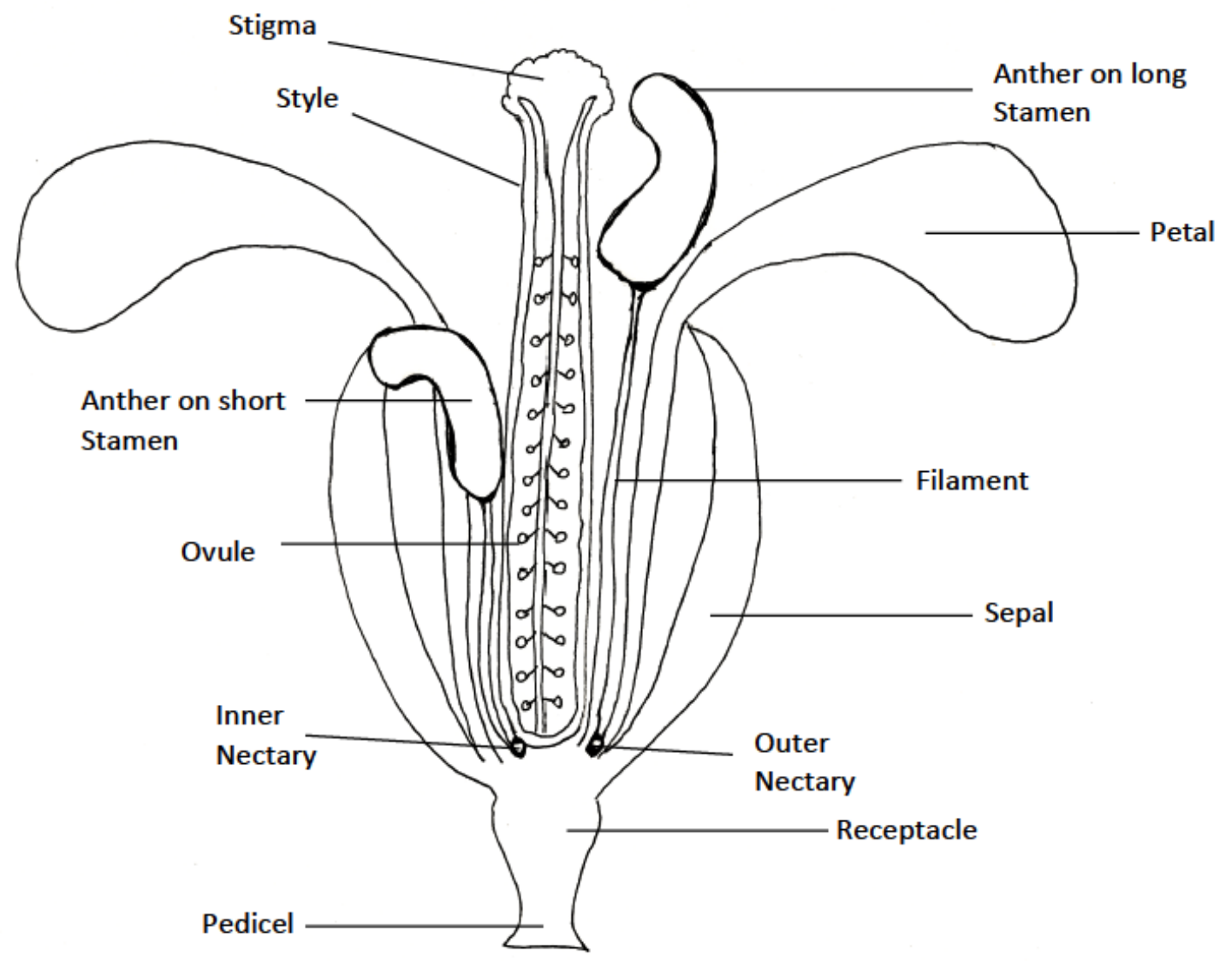

Figure 1.1. Schematic diagram of the B. carinata flower (Reneé Robinson, 2015).

Diagram was drawn using an image by Ken Bateman (http://www.fastplants.jp/pdf/activities/flowering_pollination.pdf) as a guide. 
generally a self-pollinating crop, however, it can show an average outcrossing rate of 30\% (Vollmann and Rajca, 2009).

A common technique used to improve the agronomical qualities of members of the Brassica genus is the creation of doubled haploid (DH) lines through in vitro microspore culture. The principle of androgenesis via microspore culture is to arrest the development of the pollen grains and force them to produce haploid embryos. The in vitro haploid production permits the accelerated development of homozygous lines (following chromosome doubling), allowing for the selection and evaluation of desirable genotypes (Barro et. al., 2001; Datta, 2005).

\subsection{Triticale}

Triticale is a man-made cereal crop resulting from the intergeneric cross between wheat (Triticum ssp.) as the female parent, and rye (Secale ssp.) as the male parent. The early wheat-rye hybrids were characterized by reproductive disorders such as reduced fertility and shriveled kernels. In addition, interspecific F1 hybrids were self-sterile. However, breeding programs were introduced to improve agronomical traits, and the discovery of the in vitro culture technique to rescue the aborting embryo followed by colchicine treatment (a chromosome number doubling agent) enabled the production of fertile hybrids. The first commercial triticale cultivars were made available in the late 1960’s (Oettler, 2005; Hills et. al., 2007).

There are three types of triticale based on their ploidy level, namely the tetraploid, hexaploid and octoploid varieties. However, the hexaploid hybrids $(2 n=6 x=42$;

AABBRR) are the most commercially successful. Hexaploid triticale, otherwise known 
by its scientific name $\mathrm{x}$ Triticosecale Wittmack ssp. is developed by crossing durum wheat (Triticum turgidum $\mathrm{ssp}$. durum; $2 \mathrm{n}=4 \mathrm{x}=28$; AABB) and rye (Secale cereale; $2 \mathrm{n}=$ $2 \mathrm{x}=14 ; \mathrm{RR})$. Tetraploid triticale $(2 \mathrm{n}=4 \mathrm{x}=28$; AARR, BBRR, DDRR, mixed genome $(\mathrm{AB})(\mathrm{AB}) \mathrm{RR}$ ) can be obtained by crossing hexaploid triticale with rye followed by selfing, whereas octaploid triticale $(2 \mathrm{n}=8 \mathrm{x}=56$; AABBRRDD) is produced by crossing hexaploid T. aestivum $(2 \mathrm{n}=6 \mathrm{x}=42$; AABBDD $)$ and rye. Since tetraploid triticale has never reached significance for commercial production and octoploid triticales revert to the hexaploid level, they are not normally commercially used (Oettler, 2005; Hills et. al., 2007; Lelly, 1995).

Triticale has superior agronomical qualities that arise from the cross-breeding of its parents. It has the vigor of rye, as it is more productive than other cereals under abiotic stress conditions, less susceptible to most diseases and is competitive with other cereals in terms of grain yield, and it also has many of the nutritional qualities of wheat (i.e. high starch, lipid, fiber, protein, vitamin and minerals content, low anti-nutritional content) (Hills et. al., 2007; Chapman et. al., 2005). Triticale is primarily used for animal feed, but small amounts enter the human food chain through the intentional or unintentional mixing with other grains. Since triticale is not generally used in products designed for human consumption and is competitive with other cereals with respect to grain yields, starch content and price, it can be genetically modified to produce various biopolymers, adhesives, industrial enzymes, and high strength fibers. Additionally, it is a viable platform for bioethanol production (Hills et. al., 2007; Chapman et. al., 2005).

Triticale is a cereal crop that belongs to the Triticeae tribe that is a part of the family of grasses (Poaceae) (Carvalho et. al., 2005; Grass Phylogeny Working Group, 
2001). It has a distichous spike with spikelets (containing the florets) arranged vertically on opposite sides of a central rachis. The spikelet consists of 3-5 fertile florets (with some sterile ones at the tip) enclosed by a glume. Each floret consists of a palea and a lemma with a hair like protrusion called an awn, both of which enclose and protect the reproductive parts of the flower. The flower is monoclinous, having both female (pistil consisting of the bifurcated plumose stigma, the style and the ovary) and male (3 stamens, each consisting of an anther and a filament) parts within the same flower (Figure 1.2) (Baum and Gupta, 1990). Triticale is cleistogamous and therefore predominantly a self-pollinating species, however $5 \%$ outcrossing has been reported in more advanced varieties of triticale (Yeung and Larter, 1972).

\subsection{Plant Reproductive Systems}

Plant reproduction is the process by which plants propagate. There are two types of reproduction; (1) asexual, using vegetative parts of the plants, and (2) sexual reproduction, in which two gametes (haploid cells produced by the female and male reproductive organs during meiosis) fuse to produce a diploid zygote (new offspring). When angiosperms sexually reproduce, a seed is formed from the fusion of the male and female gamete (Mauseth, 1998).

\subsection{Sexual Plant Reproduction}

\subsubsection{Female Reproductive Organs}

The female reproductive organ is called a pistil. It can consist of one carpel or a fusion of carpels (multicarpellar) collectively known as the gynoecium, and is made up of 


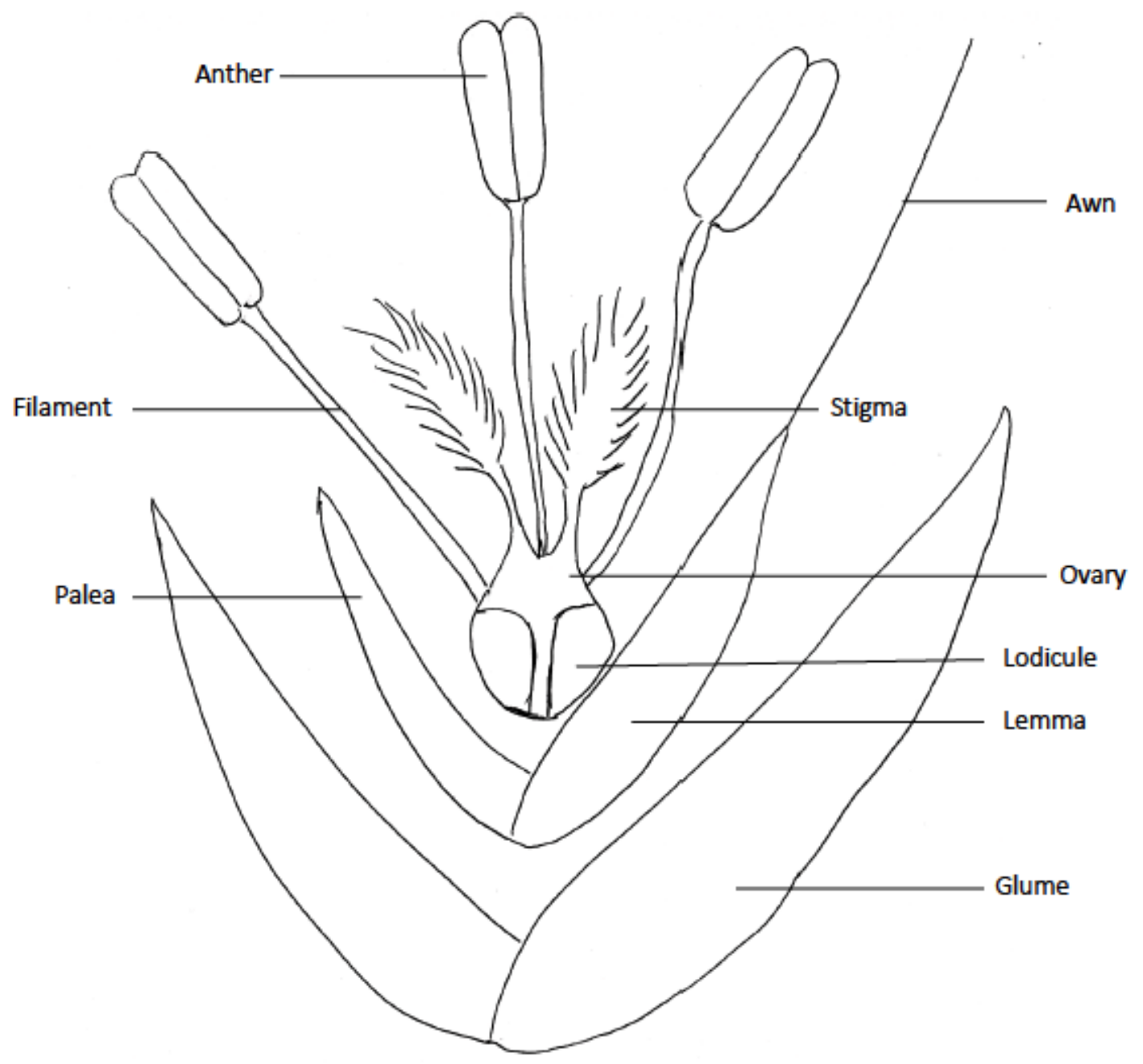

Figure 1.2. Schematic diagram of the triticale flower (Reneé Robinson, 2015). Diagram was drawn using an image found in The Encyclopedia of Grasses for Livable Landscapes (Darke, 2007) as a guide. 
3 parts; a stigma, a style, and an ovary (Mauseth, 1998). The stigma is the receptive portion of the pistil that captures the pollen grains. It can be classified into two main groups: stigmas that secrete a fluid (exudate) consisting of proteins, lipids and carbohydrates are called wet stigmas, whereas stigmas without the exudate are called dry stigmas. Dry stigmas are further divided into (1) plumose stigmas (characteristic of the grasses) that are covered by a discontinuous cuticle with receptive cells dispersed on multiseriate branches and are adapted to collect wind-dispersed pollen, and (2) papillate stigmas (characteristic of the Brassicacea), that consist of a dome of epidermal papillae covered by a continuous cuticle possessing a few patches of waxes, and are usually pollinated by pollinators. A membrane-like layer of protein called the pellicle sits on the cuticle of dry stigmas and acts as the first site of molecular contact between the stigma and pollen grains (Cresti and Tiezzi, 2011; Hiscock and Allen, 2008; Gaude and Dumas, 1986; Heslop- Harrison and Shivanna, 1977).

The style connects the stigma to the ovary and includes the transmitting tract in which the pollen tube grows to get to the ovary. The ovary contains the ovules. Ovules have a short stalk called a funiculus that attaches the ovule to the ovary wall. The ovules have a central mass of parenchyma called a nucellus that surrounds the embryo sac. Two thin sheets of cells called integuments surround almost the entire surface of the nucellus leaving a small opening (micropyle) that gives the sperm cells access to the embryo sac and the egg cell within (Hiscock and Allen, 2008; Mauseth, 1998). 


\subsubsection{Male Reproductive Organs}

The male reproductive organ of the flower is frequently referred to as the stamen. The stamens, collectively known as the androecium, contain two parts; the anther (where the pollen is produced) and the filament (the stalk supporting the anther). Within the anther, microsporocytes enlarge and prepare for meiosis. Two rounds of meiosis (I and II) occur to produce a haploid cell with four nuclei that become microspores within a tetrad enclosed in callose. The four microspores continue to expand and are released from the tetrad. Subsequently, the microspore nucleus undergoes mitosis I to produce bicellular pollen having a vegetative cell and a generative cell. The generative cell then undergoes a second round of mitosis (mitosis II) to produce two sperm cells. At this stage, the pollen grain contains three cells and is thus called tricellular pollen. In $70 \%$ of angiosperms, bicellular pollen is released from the anthers and the second round of mitosis occurs within the pollen tube. However, mitosis II occurs pre-pollen release in the remaining 30\%, including triticale and B. carinata (Mauseth, 1998; Lora et. al., 2009, Edlund et. al., 2004).

\subsubsection{Pollen Dissemination}

In angiosperms, mature pollen grains shed during anthesis can be disseminated via wind, as observed in the Poaceae, or through pollinators (insects, birds and mammals), as observed in the Brassicaceae (Kudo, 2003; Friedman and Barrett, 2009). Over the past 120 million years, flowers have adapted to facilitate their respective pollination mechanisms (Mauseth, 1998). In plants that utilize pollinators to facilitate pollen dissemination like in the Brassicaceae, the flowers contain brightly colored petals, 
are scented and produce nectar. The pollen grains are usually large and sticky, and do not become airborne readily. In wind-pollinated species like the Poaceae, the flower is often tiny, lacks petals, fragrances, and nectar. These flowers produce a large number of pollen grains (that are light, dry and easily airborne) in order to increase the chances of a compatible pollen grain landing on a compatible stigma. Large feathery stigmas are adaptive features to increase the stigmatic surface area for pollen capture (Mauseth, 1998; Treu and Emberlin, 2000).

Pollination is also aided by the growth pattern of the plant population as wind pollinated species normally grow as dense populations in rangelands or forests, and species that are widely scattered, rely on pollinators (Mauseth, 1998).

\subsubsection{Pollen-Stigma Interactions}

The pollen is released from the anthers in a partially dessicated state. Once the pollen grain lands on the stigma, it adheres to the stigma and pollen recognition occurs. If the interaction is compatible, water flows from the stigma to the pollen grain rehydrating the metabolically inactive pollen grain. The pollen grain germinates on the surface of the stigma and a pollen tube emerges out of the pollen grain. The pollen tube carrying the two sperm cells grows through the stigma cell wall, down the style and to the ovules contained within the ovary. The pollen tube penetrates the micropyle of the ovule and ruptures to release the two sperm cells into the embryo sac. One sperm cell fertilizes the egg, forming the diploid zygote, whereas the other sperm cell fertilizes the two polar nuclei to form the endosperm, which provide nutrients for the growing embryo (Mauseth, 1998; Lord and Russell, 2002; Boavida et. al., 2005). Pollen-stigma interactions can 
therefore be divided into five stages: (1) pollen capture and adhesion, (2) pollen recognition, (3) pollen hydration, (4) pollen germination, and (5) pollen tube growth. In dry stigmas, pollen capture and adhesion, recognition and hydration are highly regulated processes (Dresselhaus and Franklin-Tong, 2013). Despite their importance, knowledge of the detailed molecular mechanisms involved in these stages remains very limited.

Angiosperms can either display self-compatibility (SC), promoting inbreeding, or self-incompatibility (SI), promoting outcrossing and genetic diversity (Lord and Russell, 2002; Boavida et. al., 2005), and species can range from being fully to only partially selfincompatible (Willson, 1982). There are two types of SI systems: (1) sporophytic selfincompatibility (SSI) as observed in the Brassicaceae and (2) gametophytic selfincompatibility (GSI) as observed in the Poaceae (Lord and Russell, 2002; Baumann et. al., 2000). SC systems are not well characterized, however in the Brassicaceae the SI system has been extensively studied (Dixit and Nasrallah, 2001). Brassicaceae SSI involves the small pollen coat protein $S$-locus cysteine rich/S-locus protein 11 (SCR/SP11) and its receptor, the stigma-localized $S$ Locus Receptor Kinase (SRK). These proteins are encoded by two tightly linked and co-evolving polymorphic alleles of a single $S$-locus, called $S$ haplotypes. The SCR/SP11 ligand interacts with the stigmatic membrane localized SRK of the same S haplotype, activating it, leading to a signaling cascade that results in the rejection of self-pollen on the stigmatic surface (Chapman and Goring, 2010). Within the Brassica ssp., the intracellular signaling cascade includes a plasma membrane localized protein kinase called the $M$ Locus Protein Kinase (MLPK) that is phosphorylated by SRK. It is proposed that MLPK and SRK function in a complex to activate the downstream signaling protein Arm-Repeat Containing-1 (ARC1) 
E3 ubiquitin ligase that targets substrates for ubiquitination. Targets of ARC1 include key players of the basal compatibility pathway, namely the EXO70A1 (required for membrane vesicular docking) and glyoxalase 1 (GLO1) (detoxifies methylglyoxal in the stigmatic cytoplasm). The ubiquitinated proteins are then sent to the $26 \mathrm{~S}$ proteasome for degradation (Doucet et. al., 2016).

In the Poaceae, GSI is a multi-locus system that is controlled gametophytically by the polymorphic $S$ and $Z$ loci. A pollen grain is incompatible when the $S$ and $Z$ alleles of the pollen match that of the recipient pistil (Klaas et. al., 2011). Not much is known about the identity of the gene products, however it has recently been concluded that a DUF247 protein acts as the pollen component of the $S$ locus on linkage group (LG) 1 in perennial ryegrass. Another DUF247 gene found in perennial ryegrass and a neighboring Ubiquitin-Specific Protease (USP) gene found in both perennial ryegrass and rye, located on LG2, were implicated as male and female components of the $Z$ locus. Additionally, unlinked self-fertility loci have been identified to be located in LGs 3 and 6 (rye), and 5 (rye and perennial ryegrass) (Thorogood et. al., 2017; Shinozuka et. al., 2010; Baumann et. al., 2000).

\subsection{Proteomics}

Proteins are the cellular building blocks that directly assert the potential function of genes. Proteomics is the study of the proteome, which is defined as the entire protein complement of cells, tissues or organisms (Yates et. al., 2009). The proteome however, is affected by numerous events such as RNA processing, RNA stability control, protein translation, protein modifications and protein degradation (Soltis et. al., 2016). 
Polyploidy may also affect proteomes. Whole genome duplication events may produce copies of genes where both are retained, one is retained and the other lost or silenced, or where one copy is retained while the other diverges and takes on a new function. Furthermore, new patterns of gene expression coupled with alternative splicing may provide transcripts that could give rise to novel proteins. Within a polyploid genome, duplicate loci may undergo any combination of the aforementioned events resulting in a mosaic of possible outcomes that may consequently affect the proteome (Soltis et. al., 2016). Studies further elucidating the consequences of polyploidy on the proteome however, remain in their infancy.

Proteomics can encompass many applications including but not limited to protein identification, modification, quantification and localization (Yates et. al., 2009). Proteomic studies can be used to elucidate differences in protein abundance as well as global and organellar proteomic elucidation (Abdallah et. al., 2012).

There are numerous approaches to proteomics and they can be classified as gelbased or gel-free, as well as label-based or label-free, the latter two being used for quantitative proteomics (Abdallah et. al., 2012; Zhang et. al., 2013). All of these approaches will be discussed below.

\subsubsection{Gel-based Proteomics}

Gel-based proteomics has been the main approach used in plant proteomics and there are 4 main techniques; namely one-dimensional gel polyacrylamide gel electrophoresis (1D-PAGE), two-dimensional gel polyacrylamide gel electrophoresis 
(2D-PAGE), two-dimensional difference gel electrophoresis (2D-DIGE), and Blue Native-PAGE (BN-PAGE) (Abdallah et. al., 2012).

One-dimensional PAGE relies on the separation of proteins based on their molecular weight (MW). It allows for fast determination of protein purity and is used for many applications including the comparison of the protein composition in different samples, analysis of the number and size of polypeptide subunits, immunoblotting, and as a second dimension in 2D-PAGE (Abdallah et. al., 2012). Despite its advantages, 1DPAGE does not efficiently separate different proteins of the same MW (Magdeldin et. al., 2014). Therefore another technique, 2D-PAGE, with improved resolving power that allows proteins to undergo orthogonal separation was introduced. In 2D-PAGE, proteins first undergo isoelectric focusing (IEF) based on their net charge at different $\mathrm{pHs,}$ followed by a second dimension separation based on MW. There are however numerous caveats to this approach including poor representation of low abundance proteins and high acidic/basic proteins. Furthermore, there may be co-migration of multiple proteins in a single spot and low gel-to-gel reproducibility, rendering comparative quantification difficult. With respect to gel-based quantitative proteomics, an improvement upon the standard 2D-PAGE was introduced. This new technique called 2D-DIGE allows samples to be compared to be run together on the same gel. This circumvents the protein ratio errors due to low gel-to-gel reproducibility and it relies on pre-electrophoretic labeling of samples with CyDyes (Abdallah et. al., 2012).

Blue Native-PAGE is a technique that is used to give insight into the stoichiometry of protein complexes. It involves a two-dimensional separation where protein complexes are separated under native conditions in the first dimension followed 
by separation of individual proteins within the complexes in the second dimension under denaturing conditions. BN-PAGE however is not efficient in separating small protein complexes due to the small separation distance in the first dimension. Furthermore, distinct complexes of similar molecular masses often co-migrate resulting in constitutive proteins appearing to be present in the same complex (Abdallah et. al., 2012).

Gel-based proteomics therefore suffers from some caveats; namely reproducibility, issues with protein co-migration and limitations on the ability to study certain classes of proteins. Consequently, alternative approaches such as gel-free proteomics were introduced.

\subsubsection{Gel-free Proteomics}

In gel-free proteomics, complex protein mixtures are analyzed without the use of a polyacrylamide gel. When protein characterization is based on the analysis of peptides released from proteins through proteolysis, it is called "bottom-up" proteomics, and when this analysis is performed on a mixture of proteins, it is called shotgun proteomics (Zhang et. al., 2013). In shotgun proteomics, complex peptide fractions can be resolved using multiple fractionation strategies, many of which are gel-free. Off-gel electrophoresis (OGE) is one of these fractionation strategies. OGE involves a liquid-phase peptide IEF where the separation is performed in a two-phase system with an upper liquid phase divided into 12 or 24 compartments, and a lower phase IPG strip. The sample is loaded into the wells and a voltage gradient is applied. Peptides migrate through the IPG strip until they reach their $\mathrm{pI}$ within a given compartment. Separated peptides can then be easily recovered in solution for further analysis (Abdallah et. al., 2012). 
All the other gel-free fractionation techniques are variations of high performance liquid chromatography (HPLC) and include ion-exchange chromatography (IEC), size exclusion chromatography (SEC), normal phase liquid chromatography (NP-HPLC), hydrophilic interaction liquid chromatography (HILIC), hydrophobic interaction chromatography (HIC), and reverse phase liquid chromatography (RP-HPLC) (Abdallah et. al., 2012; Aguilar, 2004; Buszewski and Noga, 2012). HPLC consists of two phases; a stationary phase and a mobile liquid phase. The liquid phase containing the peptides is passed over the immobilized phase and the peptides are separated based on their differential absorption of each solute according to their respective affinity for the immobilized stationary phase (Aguilar, 2004). In IEC, peptides are separated according to their electric charge. There are two types: cation-exchange chromatography $(\mathrm{CX})$ and anion-exchange chromatography $(\mathrm{AX})$. In $\mathrm{CX}$, negative functional groups on the stationary phase attract positively charged peptides at an acidic $\mathrm{pH}$. In $\mathrm{AX}$, positive functional groups on the stationary phase attract negatively charged peptides at a basic $\mathrm{pH}$. Strong cation/anion exchange (SCX/SAX) encompasses a strong exchanger group that can be ionized over a broad $\mathrm{pH}$ range. Peptides are separated based on their charge by either varying the salt concentration of the mobile phase or they are separated based on their pI by applying a pH gradient (Abdallah et. al., 2012).

In SEC, peptides are separated based on differences in their molecular size. The stationary phase contains gel media that consists of spherical porous particles of a certain pore size. Small molecules diffuse freely into the pores resulting in slowed movement through the column and are therefore eluted last. Conversely, larger molecules cannot enter the pores and move more quickly through the column resulting in their earlier 
elution. SEC however does not have as good a resolving power as some of the other fractionation techniques and is therefore not the preferred method for peptide separation (Aguilar, 2004).

NP-HPLC and HILIC are very similar in the sense that they both use a polar stationary phase in combination with an apolar mobile phase for peptide separation in order of increasing polarity (Aguilar, 2004). The main difference is that HILIC uses a mobile phase similar to that of RP-HPLC (albeit reverse polarity) and it therefore allows for the analysis of charged substances. In NP-HPLC, only organic substances are used as the mobile phase and charged substances are not easily separated (Buszewski and Noga, 2012).

In HIC, analytes can bind to a weakly hydrophobic stationary phase at high salt concentrations and decreasing the salt gradient in the mobile phase allows for their elution based on increased hydrophobicity (Aguilar, 2004). RP-HPLC is similar in concept to HIC in that it separates peptides based on hydrophobicity. This technique consists of a hydrophobic stationary phase (sorbent) and a mobile aqueous phase where the peptides are eluted by the addition of organic solvent to the mobile phase and are therefore eluted in order of increasing molecular hydrophobicity (Aguilar, 2004). RPHPLC is the most widespread HPLC method applied in proteomics due to the fact that it has excellent efficiency and resolving power along with reasonable elution times, and a volatile mobile phase that is compatible with the mass spectrometer. It also eliminates the need for an extra desalting step pre-mass spectrometry (MS) that may lead to sample loss (Abdallah et. al., 2012; Aguilar, 2004). 
There is currently no single method that can provide complete qualitative and quantitative information of all the proteins in a complex mixture. Multidimensional fractionation that separates peptides according to various orthogonal physiochemical properties however, can provide deep exploration of complex proteomes and low abundance proteins, and lead to much less complex fractions therefore increasing proteome coverage (Abdallah et. al., 2012). 2D-HPLC has often been performed using IEC coupled to RP (IEC/RP) chromatography, or size exclusion chromatography coupled to RP (SEC/RP), and many offline modes of these approaches are applied where the fractions are collected after elution in the first dimension and then subjected to a second dimension. Online approaches hasten the fractionation steps and allow for less sample loss due to the direct coupling of the two dimensions in one column. In multidimensional protein identification technology (MudPIT), the SCX and RP stationary phase are packed together in the same microcapillary column (Abdallah et. al., 2012).

\subsubsection{Quantitative Proteomics: Label-based vs. Label-free}

The quantification of changes in protein abundance between samples of different states or stages is a key goal in proteomics (Resing and Ahn, 2005). Quantitative proteomics allows for the relative comparison of the abundance of proteins within two or more samples and can be approached via label-based or label-free methods. Label-free methods, for example spectral counting and measuring spectral peak intensities, involve performing multiple experiments in parallel. In spectral counting protein abundance is calculated by comparing the number of MS/MS spectra for the peptides matching the same protein between each sample. With respect to spectral peak intensities, protein 
abundance is calculated by comparing the relative abundance peaks within each peptide spectrum match (PSM) belonging to a specific protein between samples (Abdallah et. al., 2012). Label-free methods are cost effective, reproducible and less prone to side reactions related to the labeling process, however, they are more susceptible to errors due to parallel sample processing and can consequently suffer from increased analytical variability (Abdallah et. al., 2012). Conversely, label-based approaches can allow for the comparison of two or more samples within the same experiment. These approaches are based on the fact that both labeled and unlabeled peptides exhibit the same chromatographic and ionization properties but can be distinguished from each other by a mass-shift signature (brought on by the label). There are two main types: metabolic labeling and chemical labeling (Abdallah et. al., 2012).

In metabolic labeling, (for example stable isotopic labeling with amino acids in cell culture i.e. SILAC) the label is introduced to the whole organism through the growth medium consequently resulting in proteins that are labeled at the time of their synthesis. Conversely in chemical labeling, proteins or peptides are labeled with a chemical reaction (Abdallah et. al., 2012). An example of a widely used chemical labeling proteomic technique is isobaric Tag for Relative and Absolute Quantification (iTRAQ) which enables researchers to compare four to eight samples in a single experiment (Yates et. al., 2009). In iTRAQ, chemical labeling is implemented after proteolytic digest and the samples are combined before chromatography and mass spectrometry (MS). The iTRAQ label consists of a reporter group, a mass balance group, and a peptide reactive group. The peptide reactive group labels the peptide $\mathrm{N}$-termini and the $\varepsilon$-amino groups of lysine side chains. The mass balance group allows all the tags in the experiment to be of the 
same mass so that differently iTRAQ-labelled peptides can appear as a single unresolved precursor at the same $\mathrm{m} / \mathrm{z}$ in the MS spectrum. Quantification occurs in the MS product ion scans after peptide fragmentation where the iTRAQ labels fragment to produce reporter ions at a low $\mathrm{m} / \mathrm{z}$ range in a silent region of the spectrum. The reporter ion intensities are then measured and compared for relative quantification of the peptide in each sample (Abdallah et. al., 2012).

\subsubsection{Tandem Mass Spectrometry}

After the peptides are fractionated using liquid chromatography, they are separated by their mass to charge $(\mathrm{m} / \mathrm{z})$ ratios using mass spectrometry (MS). MS performed in tandem is called tandem mass spectrometry or MS/MS (Kapp and Schutz, 2007). During the first MS, soft-ionization techniques such as electrospray or matrixassisted laser desorption/ionization (MALDI) are used to generate peptide ions without fragmenting the peptides (Jorrin-Novo et. al., 2014). This approach enables intact peptides to be analyzed, as the peptide masses obtained following proteolytic digestion with a specific enzyme enables identification of the proteins within that sample via a database search (Kapp and Schutz, 2007). MS/MS goes a step further by elucidating structural information of the ionized peptides and consequently allowing researchers to determine the amino acid sequence of the peptide. Intact peptides are accelerated into a collision chamber where they undergo multiple collisions with an inert gas producing fragmentation at the amide bonds. This process called Collision Induced Dissociation (CID) provides spectra characteristic of the peptide's primary structure. Peptide 
identification algorithms are then subsequently used to interpret the MS/MS data (Kapp and Schutz, 2007).

\subsubsection{Orbitrap Fusion ${ }^{T M}$ Tribrid $^{T M}$ Mass Spectrometer}

Peptide ions are analyzed using a mass spectrometer otherwise known as a mass analyzer. In the past, single mass analyzers suffered from limited throughput and dynamic range, and therefore lacked the sensitivity to analyze a majority of the observable species within a complex biological sample. Furthermore, they required large amount of starting material, extensive fractionation and lengthy analysis time (Senko et. al., 2013). Dual mass analyzers offered some improvements, but were still limited with respect to MS/MS acquisition rates and therefore still provided limited peptide identification and proteome coverage (Senko et. al., 2013). The invention of a novel tribrid mass analyzer called the Orbitrap Fusion ${ }^{\mathrm{TM}}$ Tribrid $^{\mathrm{TM}}$ aimed to improve upon some of the limitations of previous mass analyzers. The Orbitrap Fusion ${ }^{\mathrm{TM}}$ Tribrid $^{\mathrm{TM}}$ is a combination of 3 mass analyzers; namely, the quadrupole, linear ion trap (LT), and the Orbitrap mass analyzers, and therefore combines the technologies of all three. Ions that are selected in the quadrupole mass filter can either undergo high resolution analysis in the Orbitrap analyzer or high sensitivity analysis in the LT (Senko et. al., 2013). Its Tconfiguration reduces the distance between the ion source and each mass analyzer, minimizing ion losses. Furthermore, the acquisition rate is improved by precursor ion selection in the quadrupole mass filter as they transit the analyzer, and therefore circumvents lengthy waveform based isolation susceptible to space charge effects, that would otherwise occur in the LT. Selection in the quadrupole mass filter therefore 
improves the tribrids' ability to analyze low abundance species (Senko et. al., 2013).

Another way in which the acquisition rate is improved in the tribrid is through the simultaneous execution of ion processing steps from separate spectra where ions can be detected for MS/MS "1" at the same time they are being fragmented for MS/MS "2" (Senko et. al., 2013). These technological improvements have allowed for better proteome characterization than the existing technologies and require only a fraction of the starting material, less extensive fractionation, and less analysis time (Senko et. al., 2013).

\subsubsection{Bioinformatics}

Peptide identification can be challenging and it requires the use of effective, efficient, and accurate computational methods that can identify peptides and assign amino acid sequence from product ion scans that may not be straightforward (McHugh and Arthur, 2008; Kapp and Schutz, 2007). These algorithms can be classified into three categories: (1) de novo peptide sequencing, (2) peptide sequence-tags (PSTs), and (3) uninterpreted database searching (Kapp and Schutz, 2007). De novo sequencing requires a uniform ladder of sequence ions that allows the peptide sequence to be read directly from the spectrum, however it requires a high-quality spectrum with very little background noise. The PST approach also requires a high-quality spectrum or at least a ladder of sequence ions that allows three or more residues (a tag) with corresponding flanking $\mathrm{N}$ and C-terminal masses to be read directly from the spectrum. The generated PST is then used to search the peptide database for candidate peptides (Kapp and Schutz, 2007; Liu et. al., 2006). Un-interpreted database searching matches un-interpreted MS/MS spectra to theoretical MS/MS spectra of peptides derived from calculated expected mass values 
from the gas fragmentation of the peptide (chosen based on the precursor $\mathrm{m} / \mathrm{z}$ and charge state) resulting from in silico proteolysis of proteins in a sequence database. A score or probability is then assigned to each peptide to determine the best peptide spectrum match. Proteins are then identified based on the strength of the peptide evidence (Kapp and Schutz, 2007). Un-interpreted database searching is the most commonly used method as it does not require high quality spectrum for identification purposes.

\subsection{Previous Studies of the Stigma and Pollen Proteomes}

Proteins are mediators of physiological function and therefore uncovering the proteome of a tissue is paramount to fully elucidating the cellular molecular processes within that tissue (Baggerman et. al., 2005; Yates et. al., 2009). Despite their importance, the stigma and pollen proteomes of the Poaceae and the Brassicaceae have not been extensively studied. With respect to the stigma, Samuel et. al. (2011) used 2DDIGE-MS to identify 19 proteins that decreased in abundance in B. napus stigmas following self-incompatible pollination. Major categories included response to stimulus, response to stress, aerobic and cellular respiration, glycolysis, and the tricarboxylic acid cycle. Using 2D-PAGE HPLC-MS, Sang et. al. (2012) identified 67 maize stigma specific/preferential proteins. Some of the most abundant categories included defense and stress response, carbohydrate and energy metabolism, protein metabolism and folding, cell wall remodeling and metabolism, signal transduction, and photosynthesis. The only global stigma proteome analyses known to date were performed by Nazemof et. al. $(2014 ; 2016)$ who identified 2184 and 2700 triticale and B. napus proteins respectively, using 1D- PAGE HPLC-MS/MS, 2D-PAGE HPLC-MS/MS, and OGE 
HPLC-MS/MS. Some of the most abundant categories belonged to metabolic process, protein metabolic process, transport, translation, and response to stress.

There have been more proteomic studies of the pollen than the stigma, many of which were differential analyses comparing mature and germinating pollen. For example, Dai et. al. (2007) identified 186 Oryza sativa differentially abundant proteins using 2D-PAGE MS and MS/MS. Some of the most prominent categories for the proteins with increased abundance included carbohydrate and energy metabolism, wall remodeling and metabolism, and protein metabolism, whereas the most prominent categories for the proteins with decreased abundance included wall remodeling and metabolism, and carbohydrate and energy metabolism. Using 2D-PAGE MS, Zhu et. al. (2011) identified 26 differentially abundant pollen proteins in maize. Proteins that increased in abundance had a functional skew towards tube wall modification, actin cytoskeleton organization, energy metabolism, signaling, protein folding and degradation, while proteins with decreased abundance included defense-related proteins, pollen allergens and some metabolic enzymes. Sheoran et. al. (2009) used 2D-DIGE MS/MS to identify 130 B. napus differentially abundant pollen proteins. Proteins that increased in abundance in germinating pollen were primarily involved in carbohydrate metabolism, protein metabolism and cell wall remodeling, while proteins involved in ion transport decreased in abundance. Lastly, Zou et. al. (2009) identified 189 A. thaliana proteins differentially abundant between mature and germinating pollen using 2D-PAGE MS/MS and their results suggested a role for energy metabolism, stress response and cell wall modification in pollen tube growth. 
Regarding global pollen proteome studies, Dai et. al. (2006) identified $322 O$. sativa proteins using 1D-PAGE HPLC-MS/MS, 2D-PAGE MS, and 2D-PAGE MS/MS. Proteins identified had roles in signal transduction, wall remodeling and metabolism, protein synthesis, assembly and degradation, and carbohydrate and energy metabolism. Zaidi et. al. (2012) identified 22 triticale proteins released upon hydration using 1DPAGE HPLC-MS and 2D-PAGE HPLC-MS. The proteins identified belonged to a number of protein families and included expansin B proteins, group 2-5, 7 and 11 pollen allergens, polygalacturonases, glucanases, actin depolymerizing factors, profilins, cyclophilins, glutathione peroxidases, and glutaredoxins. Holmes-Davis et. al. (2005) used 2D-PAGE MS/MS, and Sheoran et. al. (2006) and Noir et. al. (2005) both used 2DPAGE MS and 2D-PAGE HPLC-MS/MS, to identify 135, 110, and 121 pollen proteins, respectively, in A. thaliana. The proteins identified in the Holmes-Davis et. al. (2005) study had a functional skew towards metabolism, energy generation and cell structure. The Noir et. al. (2005) study also identified proteins involved in metabolism and cell wall structure, but also identified cytoskeleton related proteins, and proteins involved in protein processing. In addition to the aforementioned categories, Sheoran et. al. (2006) also identified proteins involved in defense, calcium binding, and signaling. Only two gel-free proteomic global analyses have been performed to date. Grobei et. al. (2009) identified 3500 mature pollen proteins in A. thaliana. Proteins involved in metabolism, energy, protein fate, protein synthesis, cellular transport, and development were overrepresented, while cell cycle, DNA processing, transcription were among the categories that were under-represented. Finally, Chao et. al. (2016) identified 6750 proteins and 2257 phosphoproteins in maize mature pollen. Most of the proteins identified were 
involved in response to stress, carbohydrate metabolic process, cellular amino acid metabolic process, and cellular protein modification process.

In summary, most of the proteomics studies of the mature stigma and mature pollen grain to date within the Poaceae and Brassicaceae have been gel-based and focused on differential abundance in comparison to germinating pollen. All the studies only focused on either the pollen or the stigma, and most of these studies had limited proteome coverage. In order to obtain a more detailed and complete picture of the molecular factors involved in pollen-pistil interactions, gel-free studies that include and compare the proteomes of both the stigma and pollen would be valuable.

\subsection{Hypothesis}

In this study, gel-free shotgun proteomic methods were used in order to identify proteins within the pollen and stigma of triticale and B. carinata. iTRAQ was also used quantify and compare proteins from mature stigmas to that of an earlier developmental stage. We hypothesize that the mature pollen and stigma will be enriched in proteins that are important for their upcoming interactions. Furthermore, as the stigma matures, we predict that proteins immediately involved in pollen-pistil interactions are more likely to increase in abundance. We also expect to observe proteomic differences between $B$. carinata and triticale stigma or pollen proteomes given their morphological differences resulting from their distinct evolutionary adaptations. 


\section{Chapter 2}

\section{The Brassica Mature Stigma and Pollen Proteomes: Preparing}

\section{to Meet}

\subsection{Introduction}

In angiosperms, sexual reproduction requires pollination to bring together the pollen grain and the stigma (the receptive portion of the pistil). In species containing dry stigmas (lacking the viscous surface secretion of wet stigmas), such as in the Brassicaceae, the interactions between the pollen and stigma are highly regulated. Briefly, the pollen adheres to the stigma and pollen recognition occurs. If the pollen is accepted, water subsequently flows from the stigma to the partially dessicated pollen grain thus hydrating it. The pollen grain then germinates and the pollen tube that emerges penetrates the stigmatic papillar cell wall. Each phase of this process requires intricate communication at the molecular level between the pollen and stigma.

Subsequently, the pollen tube grows down the style to the ovary, where it enters the ovule and embryo sac to deliver two sperm cells for double fertilization (Lord and Russell, 2002).

Brassica carinata, more commonly known as Ethiopian mustard, is the result of a natural hybridization between Brassica nigra and Brassica oleracea. It is a member of the Brassiceae tribe belonging to the Brassicaceae family (Alemayehu and Becker, 2002; Kristal and Lampe, 2002; Rakow, 2004). B. carinata generally has elevated erucic acid and glucosinolate levels, and is therefore undesirable for human consumption. It has a high tolerance to diseases and abiotic stresses, a large seed size and high seed yield, as 
well as high protein and oil content. The high oil content of its seed and the potential uses of the remaining meal following oil extraction make it an attractive crop for biofuel production (Johnson, 2013; Getinet et. al., 1994; Alexander et. al., 2008).

Proteins are the mediators of physiological function in the cell (Yates et. al., 2009) and it is reasonable to assume that both the mature pollen and stigma will contain proteins required for their eventual meeting. Accordingly, uncovering all the proteins that are present in the mature pollen and stigma (the stage at which they interact) is crucial for understanding the molecular mechanisms required for their interactions. This is especially important given the fact that transcriptomic data can be a poor indicator of the proteome (Nazemof et. al. 2014).

Despite their critical importance to reproduction, the pollen and stigma proteomes of the Brassicaceae have not been investigated extensively and only a handful of the proteins identified have been functionally characterized within the pollen and stigma. Furthermore, the majority of proteomic studies have relied upon gel-based approaches. With regard to the stigma, two-dimensional difference gel electrophoresis (2D-DIGE) was used to identify 19 proteins that decreased in abundance in Brassica stigmas following self-incompatible pollination (Samuel et. al. 2011). The only global proteomic analysis to date used different gel-based approaches to identify 2700 B. napus mature stigma proteins (Nazemof et. al. 2016). The Brassicaceae pollen proteome has received more attention. Gel-based proteomics served to discover 130 B. napus (Sheoran et. al. 2009) and 189 Arabidopsis thaliana (Zou et. al. 2009) proteins that were differentially abundant between mature and germinating pollen. Three independent gel-based global proteomics studies in Arabidopsis further identified 135 (Holmes-Davis et. al. 2005), 121 
(Noir et. al. 2005), and 110 (Sheoran et. al. 2006) mature pollen proteins. In contrast, gel-free proteomic analyses of the mature pollen grain in Arabidopsis have identified 3500 proteins (Grobei et. al., 2009).

This study makes the largest contribution to date towards the elucidation of the complete Brassicaceae mature pollen and stigma proteomes. Gel-free shotgun proteomics of the self-compatible species $B$. carinata mature pollen and stigma revealed 7703 and 5608 proteins, respectively. In addition, an iTRAQ analysis of the developing stigma revealed 251 proteins whose abundance changed as the stigma matured.

Combined, these resources can guide further research pertaining to the development of these vital cells, as well as to the different stages of pollen-stigma interactions.

\subsection{Materials and Methods}

\subsubsection{Plant Material}

Three biological replicates of the doubled haploid B. carinata line DH080798 EM-219 (Dr. Falk, Agriculture and AgriFood Canada, Saskatoon, SK, Canada) were grown in cabinets under a 16 hour (h) day and $8 \mathrm{~h}$ night cycle, and held at a constant humidity and temperature of $70 \%$ and $22^{\circ} \mathrm{C}$, respectively. Plants were watered twice daily and fertilized bi-weekly with 20-20-20 (NPK).

\subsubsection{Tissue Collection and Microscopy}

Brassica anthers and bud sizes were measured in order to identify the lengths corresponding to the bicellular pollen (BCP) and tricellular pollen (TCP) developmental stages. In order to identify these stages, developing anthers were harvested and fixed in 
3:1 70\% ethanol/acetic acid for at least $30 \mathrm{~min}$, washed in $70 \%$ ethanol for $5 \mathrm{~min}$ and Milli-Q water for another $5 \mathrm{~min}$, and stained with 4',6-diamidino-2-phenylindole (DAPI) to a final concentration of $4 \mu \mathrm{g} \mathrm{ml}^{-1}$. The pollen nuclei were then visualized using a Zeiss Axioplan 2 fluorescence microscope (Carl Zeiss Microscopy, Jena, Germany) and images were taken with a Zeiss AxioCam digital camera (Carl Zeiss Microimaging, Jena, Germany), and the Axiovision software (Carl Zeiss Microimaging).

Stigmas at the BCP and TCP developmental stages were visualized by light microscopy. Stigma tissues and mature pollen grain were also visualized using scanning electron microscopy (SEM). Light microscopy was performed using a Zeiss Discovery V12 light microscope (Carl Zeiss Microscopy), whereas SEM was carried out with a Quanta 600 ESEM scanning electron microscope (Thermo Fisher Scientific, San Jose, CA, USA) under environmental conditions, using a low vacuum mode and a gaseous analytical detector.

Mature pollen grains (Figure 2.1a) were harvested from dehiscing anthers using vacuum suction as described (Johnson-Brousseau and McCormick, 2004). Stigmas corresponding to the BCP stage (Figure 2.1b) were harvested from floral buds between 5$6 \mathrm{~mm}$ in length containing green anthers between 2.9-3.1 mm long, whereas stigmas corresponding to the TCP stage (Figure 2.1b) (at this stage stigmas are receptive to pollen and were considered mature) were harvested from slightly opened flower buds longer than $6 \mathrm{~mm}$ and containing yellow anthers. Harvested tissues were individually inspected under a Leica Zoom 2000 dissecting microscope (Leica Microsystems, Wetzlar, Germany) for the presence of contaminating tissue before being flash frozen in liquid nitrogen and stored at $-80^{\circ} \mathrm{C}$. 
(a)
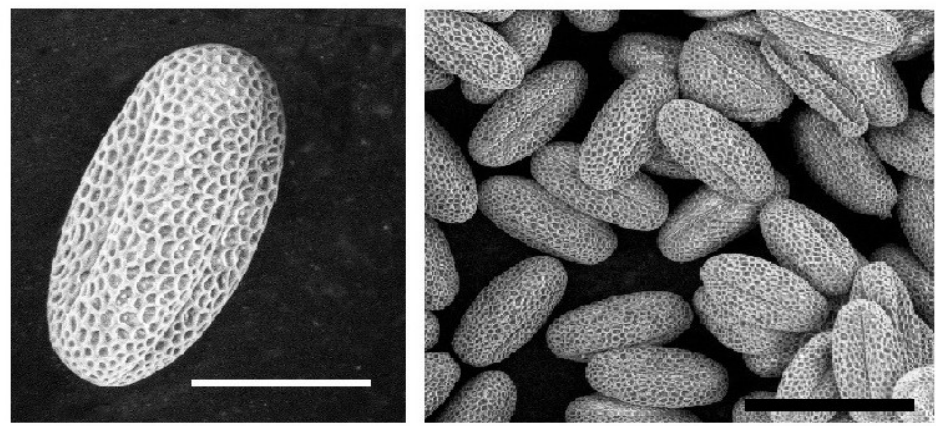

(b)

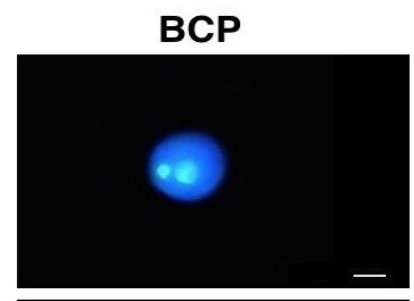

TCP
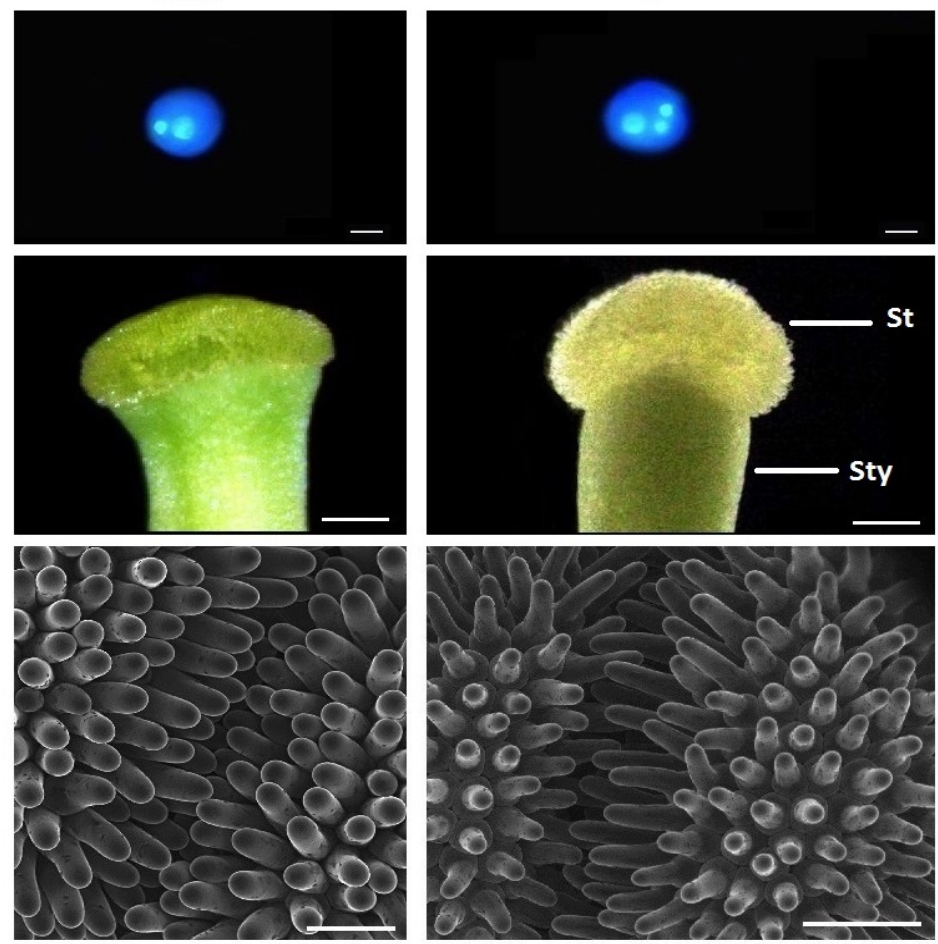

Figure 2.1. Microscopy of $B$. carinata mature pollen and stigma at the bicellular pollen (BCP) and tricellular pollen (TCP) stages of development. (a) Scanning electron micrographs of mature pollen. (b) Top row: 4',6-diamidino-2-phenylindole (DAPI)stained pollen. Middle row: Light micrographs of the upper portion of the pistil crowned by the stigma. St, Stigma; Sty, Style. Bottom row: Scanning electron micrographs of stigma papillae. Light and scanning electron microscopy photos were taken by Elizabeth Routly. Scale bars: (a) from left to right $=20 \mu \mathrm{m}, 50 \mu \mathrm{m}$; (b) top row $=20 \mu \mathrm{m}$, middle row $=250 \mu \mathrm{m}$, bottom row from left to right $=50 \mu \mathrm{m}, 100 \mu \mathrm{m}$. 


\subsubsection{Trichloroacetic Acid (TCA) Direct Protein Precipitation}

Frozen plant material (approximately 20 and $40 \mathrm{mg}$ of early and mature stigma tissues respectively, and $30 \mathrm{mg}$ of pollen) were ground and suspended in $0.25 \mathrm{ml}$ acid denaturing solution $\left(10 \%\right.$ trichloroacetic acid (TCA) in acetone pre-chilled at $-20^{\circ} \mathrm{C}$, 0.05\% dithiothreitol (DTT) added just before use). Proteins were then precipitated by incubation overnight at $-20^{\circ} \mathrm{C}$. The precipitate was then centrifuged at $4^{\circ} \mathrm{C}$ for $45 \mathrm{~min}$ at $21,100 \mathrm{x}$ g, the supernatant discarded, and the pellet washed in $200 \mu 1$ rinsing buffer (acetone pre-chilled at $-20^{\circ} \mathrm{C}, 0.05 \%$ DTT added just before use) and incubated at $-20^{\circ} \mathrm{C}$ for $30 \mathrm{~min}$. The mixture was centrifuged at $4^{\circ} \mathrm{C}$ for $20 \mathrm{~min}$ at $21,100 \mathrm{xg}$, the supernatant discarded and the pellet washed two more times in rinsing buffer before drying on ice and storage at $-80^{\circ} \mathrm{C}$ for later use. Total protein concentrations were determined with the BioRad Bradford protein assay kit (Bio-Rad, Mississauga, ON, Canada) according to the manufacturer's instructions.

\subsection{4 iTRAQ Analysis}

iTRAQ analysis was performed on proteins extracted from B. carinata stigmas at the BCP (early) and TCP (late) stages (Figure S1). Proteins (200 $\mu \mathrm{g})$ from each stage within each of three biological replicates (A, B and C) were aliquoted, split into two equal technical replicates of $100 \mu \mathrm{g}$, air-dried using the GeneVac EZ-2 solvent evaporator (SP Scientific, Warminster, PA, USA) and sent to the Plate-forme protéomique, Centre Génomique de Québec (http://proteomique.crchul.ulaval.ca/en/) for protein digestion, iTRAQ labeling, and high performance liquid chromatography (HPLC)-tandem mass spectrometry (MS)/MS as follows. 
Protein pellets were resuspended in a solution of 4.5 $\mathrm{M}$ urea and 1\% sodium deoxycholate. Equal amounts of protein, $20 \mu \mathrm{g}$ for each replicate, were used for iTRAQ labeling. Proteins were first reduced with $7.5 \mathrm{mM}$ DTT for $30 \mathrm{~min}$ at $37^{\circ} \mathrm{C}$ and then alkylated with $14.3 \mathrm{mM}$ iodoacetamide (IAA) for $20 \mathrm{~min}$ at room temperature (RT). The urea concentration was diluted to $2 \mathrm{M}$ with water and samples were digested with trypsin (Sequence Grade Modified, Promega Corporation, Madison, WI, USA) using a 1:30 ratio overnight at $37^{\circ} \mathrm{C}$. After digestion, peptides were acidified to precipitate deoxycholate and then purified with a C18 Empore filter (Sigma-Aldrich Canada Co., Oakville, ON, Canada) and lyophilized. Dried peptides were dissolved in $30 \mu 10.5 \mathrm{M}$ triethylammonium bicarbonate (TEAB) and labeled with iTRAQ label reagent (Applied Biosystems, Foster City, CA, USA). Four-plex labeling (Figure S2) was performed for 2 $\mathrm{h}$ at RT in the dark. Labeled peptides were combined in one tube and cleaned using HLB cartridges (Waters Corporation, Milford, MA, USA) and dried with a SpeedVac (Thermo Fisher Scientific, San Jose, CA, USA).

iTRAQ samples were fractionated (24 fractions) by high $\mathrm{pH}$ ( $\mathrm{pH} 10)$ reversedphase (RP) HPLC using the Agilent Technologies Inc. 1200 HPLC system (Agilent Technologies Inc., Santa Clara, CA, USA). The samples were loaded in $10 \mathrm{mM}$ ammonium bicarbonate buffer ( $\mathrm{pH} 10$ ) at $25 \mu \mathrm{l} / \mathrm{min}$ on a Zorbax Extend $\mathrm{C} 18$ microbore RR column (Agilent Technologies Inc.) and eluted with a gradient of 5-35\% of B (A: Water, $10 \mathrm{mM}$ ammonium bicarbonate, B: 90\% Acetonitrile, $10 \mathrm{mM}$ ammonium bicarbonate) for $60 \mathrm{~min}$, and then $35-70 \%$ of B in $24 \mathrm{~min}$. The fractions were pooled as described in Yang et. al. (2012). Fractions were then dried with the SpeedVac and resuspended in $0.1 \%$ formic acid for analysis by MS. For each experiment, $750 \mathrm{ng}$ of 
peptide samples were injected and separated by online RP nanoscale capillary LC (nanoLC) and analyzed by electrospray ionization (ESI) MS/MS. The experiments were performed with a Dionex UltiMate 3000 nanoRSLC chromatography system (Thermo Fisher Scientific/Dionex Softron GmbH, Germering, Germany) connected to an Orbitrap Fusion $^{\mathrm{TM}}$ Tribrid $^{\mathrm{TM}}$ mass spectrometer (Thermo Fisher Scientific) equipped with a nanoelectrospray ion source. Peptides were trapped at $20 \mu \mathrm{l} / \mathrm{min}$ in loading solvent ( $2 \%$ acetonitrile, $0.05 \%$ trifluoroacetic acid (TFA)) on a $5 \mathrm{~mm} \times 300 \mu \mathrm{m}$ C18 pepmap cartridge pre-column (Thermo Fisher Scientific/Dionex Softron $\mathrm{GmbH}$ ) for 5 min. The pre-column was then switched online with a self-made $50 \mathrm{~cm}$ x $75 \mu \mathrm{m}$ internal diameter separation column packed with ReproSil-Pur C18-AQ 3- $\mu$ m resin (Dr. Maisch, HPLC $\mathrm{GmbH}$, Ammerbuch-Entringen, Germany) and the peptides were eluted with a linear gradient from $5-40 \%$ solvent B (A: $0.1 \%$ formic acid, B: $80 \%$ acetonitrile, $0.1 \%$ formic acid) for $90 \mathrm{~min}$ at $300 \mathrm{~nL} / \mathrm{min}$. Mass spectra were acquired using the data-dependent acquisition mode using Thermo XCalibur software version 3.0.63. Full scan mass spectra (350 to $1800 \mathrm{~m} / \mathrm{z})$ were acquired in the Orbitrap using an automatic gain control (AGC) target of $4 \times 10^{5}$, a maximum injection time of $50 \mathrm{~ms}$ and a resolution of 120000. Internal calibration using lock mass on the $\mathrm{m} / \mathrm{z} 445.12003$ siloxane ion was used. Each MS scan was followed by acquisition of fragmentation MS/MS spectra of the most intense ions for a total cycle time of $3 \mathrm{~s}$ (top speed mode). The selected ions were isolated using the quadrupole analyzer in a window of $1.6 \mathrm{~m} / \mathrm{z}$ and fragmented by Higher energy Collision-induced Dissociation (HCD) with $45 \%$ of collision energy. The resulting fragments were detected by the Orbitrap at resolution 60000 with an AGC target of $1 \times 10^{5}$ and a maximum injection time of $120 \mathrm{~ms}$. Dynamic exclusion of previously 
fragmented peptides was set for a period of $20 \mathrm{~s}$ and a tolerance of $10 \mathrm{ppm}$. MS/MS peak lists (MGF files) were generated using Proteome Discoverer version 1.4.0.288 (Thermo Fisher Scientific). MGF samples were analyzed using Mascot version 2.5.2 (Matrix Science, London, UK) to search the TAX_Brassiceae_981017 database (https://www.uniprot.org/uniprot/?query=brassiceae\&sort=score) assuming trypsin digestion with two missed cleavages being allowed, a fragment ion mass tolerance of 0.025 Da, and a parent ion tolerance of 10.0 PPM. Carbamidomethylation of cysteine was specified as a fixed modification. Deamidation of asparagine and glutamine, oxidation of methionine and iTRAQ 4-plex of lysine, tyrosine and the N-terminus were specified as variable modifications.

Scaffold version 4.7.5 (Proteome Software Inc., Portland, OR, USA) was used to validate MS/MS based peptide and protein identifications. Peptide identifications were accepted if they could be established at greater than $95.0 \%$ probability by the Scaffold Local FDR algorithm. Protein identifications were accepted if they could be established at greater than $95.0 \%$ probability and contained at least 2 exclusive peptides. Protein probabilities were assigned by the protein prophet algorithm (Nesvizhskii et. al., 2003). Proteins that contained similar peptides and could not be differentiated based on MS/MS analysis alone were grouped to satisfy the principles of parsimony. Proteins sharing significant peptide evidence were grouped into clusters.

Scaffold Q+ version 4.7.5 (Proteome Software Inc.) was used to quantitate iTRAQ protein identifications. Only proteins identified (at the thresholds described above) across all replicates were used for quantification. Normalization was performed iteratively (across samples and spectra) on intensities, as described in Oberg et. al., 2008. 
Medians were used for averaging. Spectra data were log-transformed, pruned of those matched to multiple proteins and those missing a reference value, and weighted by an adaptive intensity weighting algorithm. Statistically significant differentially abundant proteins were determined by applying the Mann-Whitney test with unadjusted significance level $\mathrm{p} \leq 0.05$ corrected by Benjamini-Hochberg to $\mathrm{p} \leq 0.03491$. Proteins showing $\mathrm{a} \geq 1.5$ fold difference in abundance were considered differentially abundant.

\subsubsection{Global Mass Spectrometry Analysis}

Global analyses were performed on proteins extracted from mature $B$. carinata stigmas and pollen grain (Figure S3). Approximately $1 \mathrm{mg}$ of the mature stigma and pollen proteins were each resuspended in $6 \mathrm{M}$ urea, $50 \mathrm{mM}$ Tris $(\mathrm{pH}$ 8) to a final concentration of $6 \mu \mathrm{g} \mathrm{ml}^{-1}$. The samples were agitated at RT at $650 \mathrm{RPM}$ for $1 \mathrm{~h}$ and then placed on a rotor for $5 \mathrm{~h}$ for further agitation. The proteins were then reduced by the addition of DTT to a final concentration of $10 \mathrm{mM}$ and then agitated at $56{ }^{\circ} \mathrm{C}$ at $650 \mathrm{RPM}$ for 45 min. Alkylation of the proteins was performed by the addition of 0.4 M IAA solution in $50 \mathrm{mM}$ ammonium bicarbonate to a final concentration of $14 \mathrm{mM}$. The samples were then agitated on a rotator for $30 \mathrm{~min}$ at RT in the dark. Trypsin was added to the samples and the proteins digested according to manufacturer's instructions (Promega Corporation) with agitation at $750 \mathrm{RPM}$ at $37^{\circ} \mathrm{C}$ overnight. The tryptic digests were adjusted to $\mathrm{pH} 10$ with ammonium formate and $1 \mathrm{ml}$ for each sample was injected individually and separated offline into 60 fractions via high $\mathrm{pH}(\mathrm{pH}$ 10) RP-HPLC using a C18 Hypersil gold column (Thermo Fisher Scientific). The experiments were performed with the ÄKTA purifier (Amersham Biosciences, Little Chalfont, UK) and the 
peptides were eluted with a linear gradient from 4-40\% solvent B (A: water, $20 \mathrm{mM}$ ammonium formate $\mathrm{pH}$ 10; $\mathrm{B}$ : acetonitrile (ACN), $20 \mathrm{mM}$ ammonium formate $\mathrm{pH} 10$ ), at a flow rate of $1 \mathrm{ml} / \mathrm{min}$. The fractions were then concatenated into 20 fractions by combining fractions $14,34,54,74 ; 15,35,55 \ldots$ and the pooled samples were then airdried in a GeneVac EZ-2 solvent evaporator (SP Scientific) and sent to the Plate-forme protéomique, Centre Génomique de Québec (http://proteomique.crchul.ulaval.ca/en/) for inline low pH RP-HPLC MS/MS. Peptide samples $(1 \mu \mathrm{g})$ were injected and separated by online RP nanoLC and analyzed by ESI MS/MS as described above with the exception that the peptides were eluted in 35 min and the linear ion trap was used to detect the peptide fragments after HCD. The fragments were detected in a rapid scan rate with an AGC target of $1 \times 10^{4}$ and a maximum injection time of $50 \mathrm{~ms}$.

A second global MS analysis was performed on mature stigma proteins. Aliquots $(10 \mu \mathrm{g})$ from each of the 6 mature B. carinata stigma protein samples not used for the iTRAQ analysis, that had already been sent to the Plate-forme protéomique, Centre Génomique de Québec (http://proteomique.crchul.ulaval.ca/en/), were washed 3 times with $50 \mathrm{mM}$ ammonium bicarbonate buffer using an Amicon $3 \mathrm{kDa}$ column. After elution, the samples were vacuum-dried and kept at $-20^{\circ} \mathrm{C}$ until trypsin digestion. Proteins were solubilized in $25 \mu 150 \mathrm{mM}$ ammonium bicarbonate, $1 \%$ sodium deoxycholate and heated at $95^{\circ} \mathrm{C}$ for $5 \mathrm{~min}$. Two micrograms of each sample was reduced with DTT $(0.2 \mathrm{mM})$ at $37^{\circ} \mathrm{C}$ for $30 \mathrm{~min}$ and alkylated with IAA $(0.9 \mathrm{mM})$ at $37^{\circ} \mathrm{C}$ for $20 \mathrm{~min}$. Finally, trypsin $(1 \mu \mathrm{g})$ was added and the samples were incubated overnight at $37^{\circ} \mathrm{C}$. The trypsin reaction was stopped by acidification with a solution of $3 \%$ acetonitrile, $1 \%$ TFA, and $0.5 \%$ acetic acid. The peptide samples were combined, 
purified on a stage tip (C18) and vacuum-dried before MS injection. Peptides were resuspended into $0.1 \%$ formic acid for analysis by MS. Peptide samples $(1 \mu \mathrm{g})$ were injected and separated by online RP nanoLC and analyzed by electrospray mass spectrometry (ESI MS/MS) as mentioned above with the exception that the peptides were eluted in $270 \mathrm{~min}$ and the linear ion trap was used to detect the peptide fragments after HCD. The fragments were detected in a rapid scan rate with an AGC target of $1 \times 10^{4}$ and a maximum injection time of $50 \mathrm{~ms}$.

All MS/MS peak lists (MGF files) were generated as above and all global experiments were analyzed using Mascot version 2.5.1 and X! Tandem (The GPM, https://thegpm.org; version CYCLONE (2010.12.01.1)). Mascot and X! Tandem were used to search the TAX_Brassiceae_981017 database and the TAX_Viridiplantae_33090 database (https://www.uniprot.org/uniprot/?query=viridiplantae\&sort=score) assuming trypsin digestion with two missed cleavages being allowed. They were searched with a fragment ion mass tolerance of $0.60 \mathrm{Da}$ and a parent ion tolerance of $10.0 \mathrm{ppm}$. Carbamidomethylation of cysteine was specified as a fixed modification. Oxidation of methionine, deamidation of asparagine and glutamine, dehydration of the N-terminusglutamic acid to pyro-glutamate of the N-terminus, and ammonia-loss of the N-terminusglutamine to pyro-glutamate of the $\mathrm{N}$ terminus were specified as variable modifications. Scaffold version 4.7.5 (Proteome Software Inc.) was used to validate MS/MS based peptide and protein identifications as described above. Proteins with a single peptide match were validated as previously described (Nazemof et. al. 2014). 


\subsubsection{General Data Analysis}

Proteins identified from the various searches were combined as follows. The proteins identified from searches using the Brassiceae database were combined to nonBrassiceae proteins identified exclusively from searches using the Viridiplantae database. For the stigma, proteins identified in at least one sample from the iTRAQ analysis were also combined with proteins identified from the global Brassiceae and Viridiplantae searches provided that their matching Arabidopsis orthologs were absent from the list of Arabidopsis orthologs of proteins from the initial Brassiceae and Viridiplantae searches.

Proteins were annotated based on the gene ontology (GO) classification system (www.geneontology.org) using the annotation software Blast2Go version 4.1.9 (www.blast2go.com, BioBam, Valencia, Spain). BlastP was performed against the NCBI non-redundant protein sequence (nr) database using the top 20 hits at an expectation value (E) threshold of $\leq 1 \times 10^{-25}$. The annotation step was configured with an annotation cutoff of 55 and $\mathrm{GO}$ weight of 5. Annotations were also retrieved from domain/motif information in a sequence-wise manner using the Blast2Go InterProScan and merged with existing annotations. Finally, annotations were augmented using the Blast2Go Annex program. The annotations were categorized based on their Biological Processes. Proteins with no significant matches and proteins matching known proteins or domains but without an associated Biological Process were grouped together under the category "Unclassified and Unknown". To identify the Arabidopsis thaliana orthologs, a BLAST was performed with the protein sequences against The Arabidopsis Information Resource (TAIR) protein database using the top hit at an E-threshold of $\leq 1 \times 10^{-5}$ and annotated manually. For enrichment analyses, Fisher's exact term enrichment analysis 
was performed on Arabidopsis orthologs at an E-threshold of $\leq 1 \times 10^{-25}$ using the AmiGo2 online tool (http://amigo.geneontology.org/amigo) with the background set as the PANTHER Biological Process GO annotated Arabidopsis thaliana proteome which contained 27060 proteins (Mi et. al., 2017). The p-values were adjusted for multiple tests with the Benjamini-Hochberg procedure, and only terms with an adjusted p-value of $<0.05$ were considered to be statistically enriched or under-represented.

\subsubsection{String Network Analysis}

Protein-protein interaction network building was performed with Arabidopsis orthologs using the online tool STRING version 10.5 (https://string-db.org, Szklarczyk et. al., 2017). Evidence for protein-protein interaction was taken from known experimental interactions, co-expression analysis, and known pathways of the input proteins or their orthologs in other species.

\subsection{Results and Discussion}

\subsubsection{Functional Classification of B. carinata Mature Pollen and Stigma Proteins}

In a compatible reaction, a vast number of proteins are enlisted in the processes of pollen adhesion, recognition, hydration, germination and tube growth, with many of the pollen and stigma proteins working towards the same objectives. Accordingly, in addition to housekeeping proteins, the mature pollen and stigma proteomes would be expected to include proteins having distinctive, as well as shared functions. In total, 7703 and 5608 proteins were identified from the analysis of the $B$. carinata mature stigma and pollen proteomes, respectively, and to our knowledge these represent the most 
comprehensive Brassicaceae stigma and pollen proteomic datasets to date (Table S2.1). To investigate these proteomes, proteins were first annotated based on gene ontology (GO) and allocated to 14 broad biological process categories. The proteins identified in the mature stigma (Table S2.2) and pollen (Table S2.3) displayed similar overall global functional distribution patterns with most proteins falling into 6 categories: Carbohydrate Metabolic Process, Metabolic Process, Protein Metabolic Process, Regulation of Cellular Process, Response to Stress, and Transport (Figure 2.2). This protein distribution trend amongst the 14 broad categories was comparable to that obtained for the B. napus mature stigma proteome (Nazemof et. al., 2016), even though approximately 3 times as many stigma proteins were identified in this report. The similarities between these different datasets may reflect to some extent a dominating effect of their common proteins such as housekeeping proteins.

The protein datasets were then analyzed to uncover the biological processes that were enriched or under-represented in the stigma or pollen with respect to the entire plant. The stigma proteome had 22 main categories that contained enriched subcategories, with Development and Growth, Gene Expression, Nucleobase-containing Compound Metabolism, Protein Metabolism, Response to Stimulus, RNA Metabolism and Transport being prevalent, whereas Transcription was under-represented (Figure 2.3). The pollen proteome had 17 main categories that contained enriched sub-categories, with Development and Growth, Nucleobase-containing Compound Metabolism, and Transport predominating, while Defense Response and Transcription were under-represented (Figure 2.3). The proteins and GO sub-categories corresponding to each category are shown in Table S2.4 (stigma) and Table S2.5 (pollen). Generally, the enriched and 


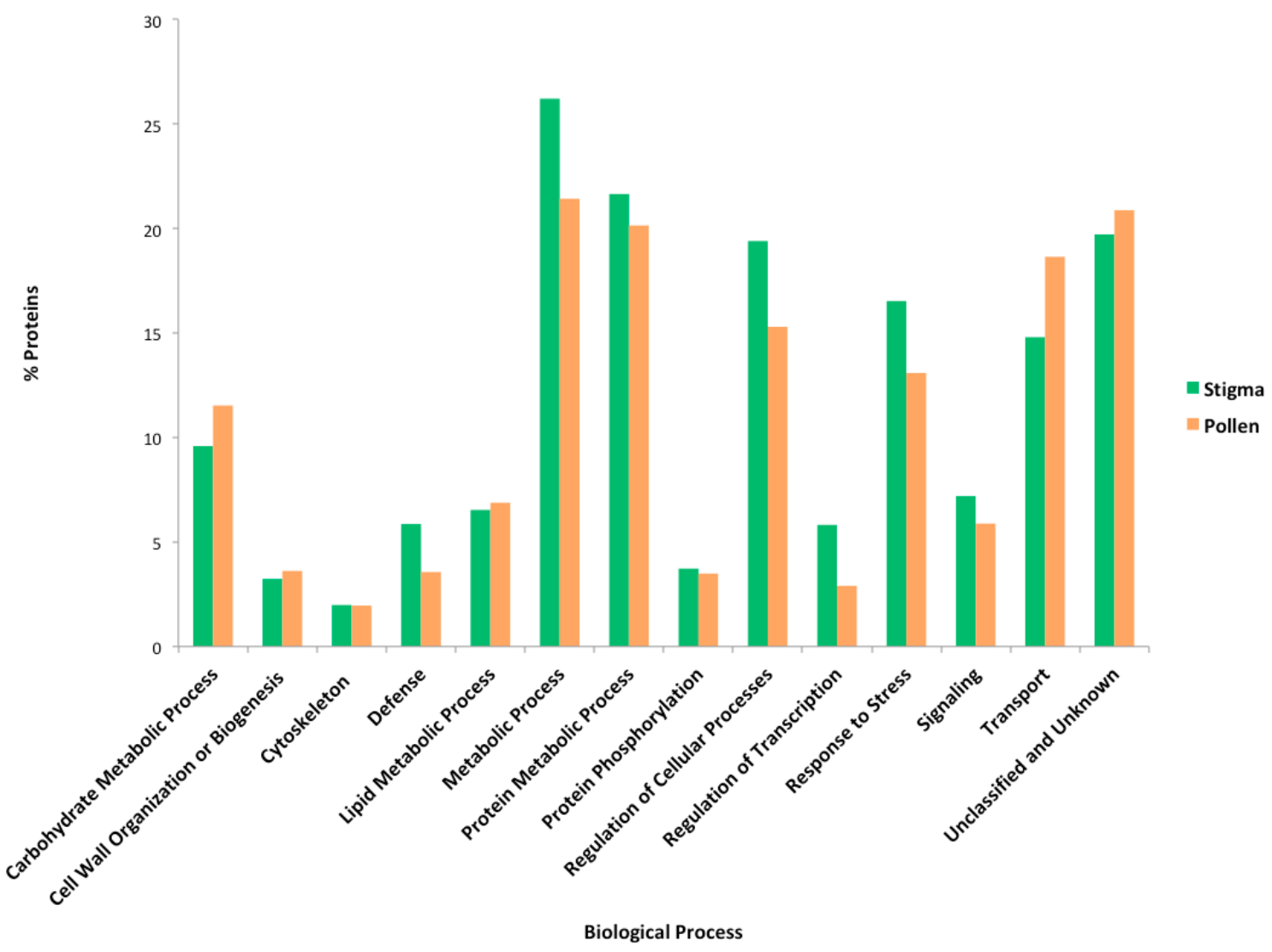

Figure 2.2. Comparison of the functional distribution of the $B$. carinata mature stigma and pollen proteomes. 


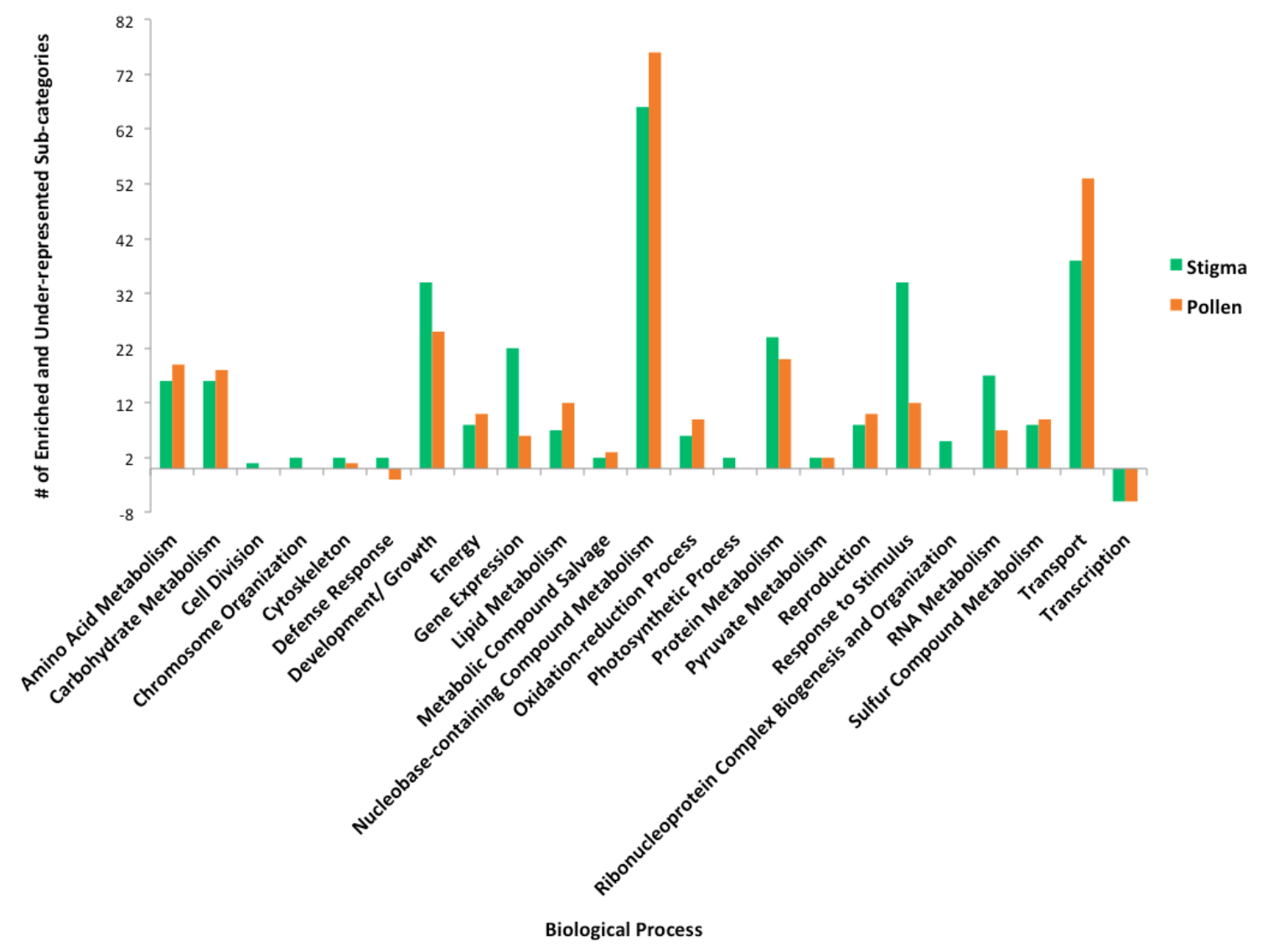

Figure 2.3. Comparison of the number of enriched and under-represented functional subcategories of the $B$. carinata mature stigma and pollen proteomes. 
under-represented categories observed for the $B$. carinata pollen proteome were in good agreement with those of a previous analysis of the Arabidopsis pollen proteome, where Metabolism, Energy, Protein Fate, Protein Synthesis, Cellular Transport and Development were over-represented, while categories like Cell Cycle, DNA Processing and Transcription were under-represented (Grobei et. al., 2009).

The stigma proteome had noticeably more enriched sub-categories than the pollen in Cell Division, Chromosome Organization, Development and Growth, Gene Expression, Photosynthetic Process, Protein Metabolism, Response to Stimulus, Ribonucleoprotein Complex Biogenesis and Organization, and RNA Metabolism (Figure 2.3). Conversely, the pollen proteome had clearly more enriched sub-categories in Lipid Metabolism, Nucleobase-containing Compound Metabolism, and Transport (Figure 2.3). It is evident that significant differences exist between the pollen and stigma proteomes, especially when looking at the number of sub-categories unique to each dataset (Figure 2.4) and some of the main categories will be discussed below.

Although the Development and Growth category contained many enriched subcategories in both the stigma and the pollen proteome datasets in comparison to the entire plant (Figure 2.3 and 2.4), the unique stigma-enriched sub-categories included Root Development, Phyllome Development, Post-embryonic Development and Plant Epidermal Cell Differentiation (Table S2.4), whereas the unique pollen-enriched subcategories included Pollen Development, Pollen Tube Development, and Cell Tip Growth (Table S2.5). Some of the shared sub-categories within the pollen and stigma proteomes pertained to Cell Development, Cell growth, Cell Morphogenesis, Developmental 


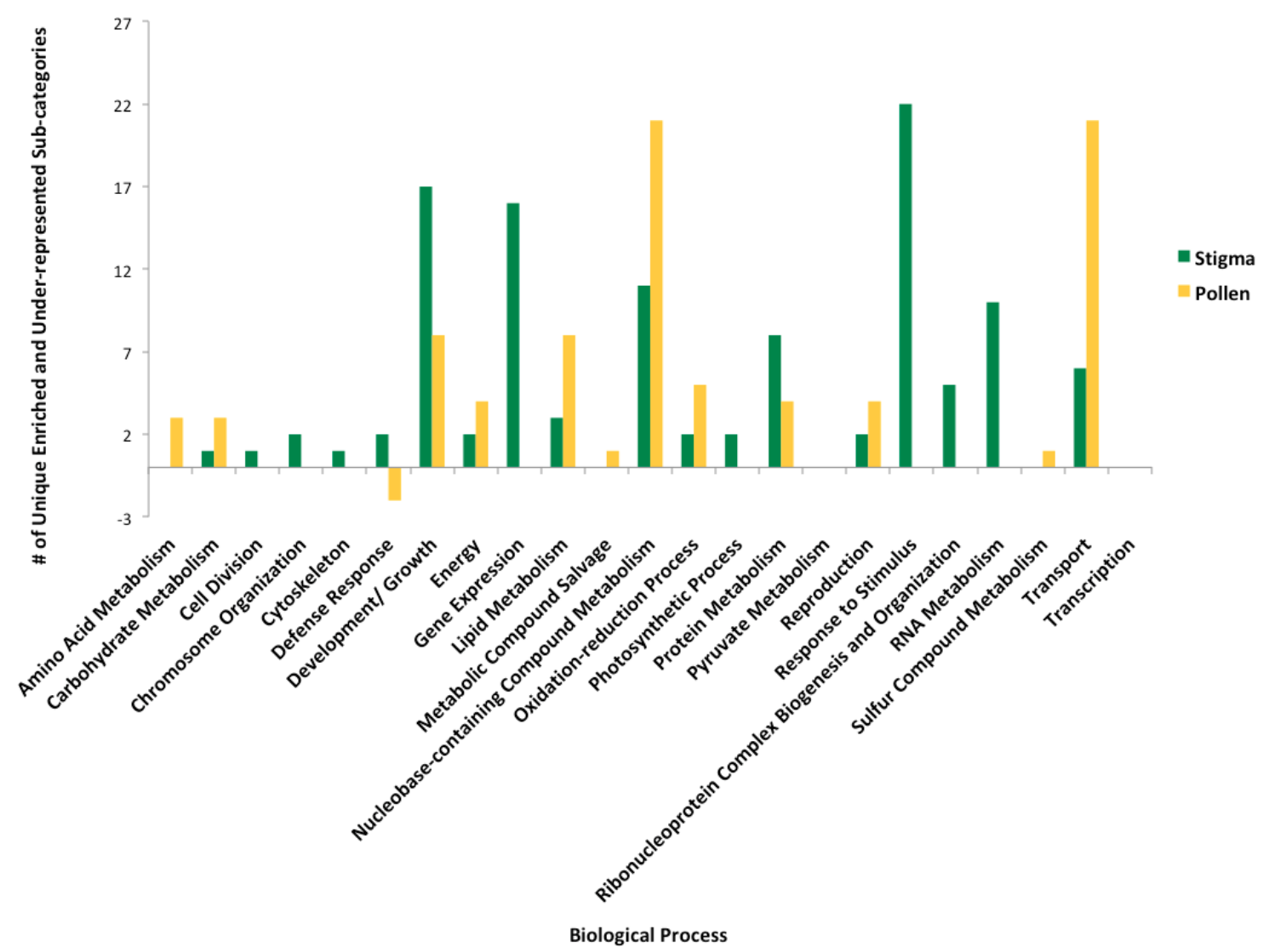

Figure 2.4. Comparison of the number of unique enriched and under-represented functional sub-categories of the B. carinata mature stigma and pollen proteomes. 
Process Involved in Reproduction and Embryo Development. More than $90 \%$ of the stigma and pollen proteins within the Development and Growth category (Tables S2.4 and S2.5) were also found in other categories indicating that most of the Development and Growth proteins are part of multiple biological processes and could have multiple roles.

RNA metabolism and Gene Expression contained numerous sub-categories that were enriched in both the stigma and the pollen grain in comparison to the rest of the plant (Figure 2.3). However, when compared to the pollen, the stigma displayed more unique enriched sub-categories involved in Gene Expression, Ribonucleoprotein Complex Biogenesis and Organization, and RNA metabolism (Figure 2.4). Unlike the pollen grain, the stigma is still metabolically active at maturity and accordingly 187 proteins in these sub-categories were involved in RNA Processing (e.g. pre-mRNA splicing factors, exosome complex components), Ribonucleoprotein Complex Assembly, as well as in Chromatin Remodeling, DNA Methylation, and Histone Modification (Table S2.4). With regard to the pollen grain, the enriched sub-categories included posttranscriptional processes like mRNA Catabolic Process and Post-Transcriptional Regulation of Gene Expression (Table S2.5). Similar results were reported with Arabidopsis where RNA synthesis was found to be under-represented, although there was a significant over-representation of post-transcriptional processes, in the mature pollen grain indicating that the processing and translation of stored transcripts is one of the first events triggered at germination and during initial pollen tube growth (Grobei et. al., 2009). 
While the Brassicaceae pollen grain is essentially inert and covered by a thick protective cell wall, the stigma, as the receptive portion of the pistil, needs to be responsive to the pollen grain as well as the invading pollen tube, which essentially creates a wound and likely increases susceptibility to pathogens. Accordingly, although Response to Stimulus contained enriched sub-categories in both the pollen and stigma proteomes (Figure 2.3), the stigma contained the highest number of unique enriched subcategories, and 6 of them were related to defense- and stress-related processes (Figure 2.4, Table S2.4). The stigma also had approximately $2 \mathrm{X}$ more proteins involved in the response to light (e.g. COP9 signalosome subunits and photosystem II proteins). The majority of the pollen-enriched sub-categories in Response to Stimulus pertained to responses to abiotic stimuli presumably in preparation for the possible exposure to abiotic stresses after release from the anthers (Table S2.5).

The Transport category contained numerous enriched sub-categories in both the stigma and the pollen grain in comparison to the rest of the plant (Figure 2.3). As discussed below, this is expected since stigma vesicular trafficking is needed to carry water for pollen hydration, as well as nutrients, enzymes and other materials to the pollen contact sites in order to facilitate pollen germination and tube growth. In the stigma, it therefore plays more of a facilitating role. Conversely, in the pollen, the purpose of vesicular trafficking is to drive rapid pollen tube tip growth at the apex (Goring et. al., 2017; Cheung and $\mathrm{Wu}, 2008)$.

Lipid metabolism contained enriched sub-categories in both the stigma and the pollen grain proteomes in comparison to the rest of the plant (Figure 2.3), however, the stigma contained substantially more proteins with a potential role in cuticle development 
including 3-ketoacyl-CoA synthases such as KCS4, KCS13 and KCS19 (Hegebarth and Jetter, 2017), cytochrome P450s like CYP86A2 (Molina et. al., 2008; Xiao et. al., 2004), CYP86A4 (Li-Beisson et. al., 2009), as well as CYP86A8 (Schnurr et. al., 2004), ECERIFERUM (CER) proteins such as CER10 (Zheng et. al., 2005), CER2 (Haslam et. al., 2012) and CER4 (Rowland et. al., 2006) to name just a few (Tables S2.4 and S2.5). Nucleobase-containing Compound Metabolism possessed the highest number of enriched sub-categories in both pollen and stigma (Figure 2.3). Nucleobase-containing compounds are a part of a variety of processes necessary for pollen tube growth including the production of nucleotide sugars that are the building blocks for cell wall polysaccharides, ion homeostasis via cyclic nucleotide-gated ion channels, ATP generation for energy, as well as the generation of GDP/GTP-containing molecules for signaling and cytoskeleton dynamics (Zrenner et. al., 2006; Song et. al., 2009; Yue et. al., 2014; Cheung and $\mathrm{Wu}, 2008)$. The pollen exhibited twice as much uniquely enriched Nucleobase-containing Compound Metabolism sub-categories than the stigma (Figure 2.4). Most of the unique sub-categories in the pollen pertained to processes involved in pyrimidine-containing compound metabolism, especially the biosynthesis of uridine monophosphate (UMP), a precursor for uridine diphosphate (UDP) and UDP-sugar used in the synthesis of cell wall polysaccharides required for cell wall remodeling and biosynthesis during pollen tube growth (Zrenner et. al., 2006).

Many of the proteins found within the categories described above will be discussed below in the context of pollen and stigma function and interactions. 


\subsubsection{Differential Protein Abundance during B. carinata Stigma Maturation}

The maturing stigma could be expected to accumulate proteins that will be required in the ensuing interaction with the pollen grain, and maybe also to reduce the abundance of proteins that were specifically involved in processes relating to its maturation. iTRAQ analysis was performed to identify differentially abundant proteins (DAPs) during $B$. carinata stigma maturation and out of the 3170 proteins identified, 251 were considered to be differentially abundant. There were 154 proteins that increased in abundance (Table S2.6), while 97 proteins decreased in abundance (Table S2.7). Preliminary analysis of differentially expressed B. carinata transcripts obtained from the same stigma developmental stages revealed that $73 \%$ of the proteins that increased in abundance (matching the corresponding transcript at an e-value of 0.0 ) were found in the group of up-regulated transcripts, while $60 \%$ of the proteins that decreased in abundance, were found in the down-regulated transcripts group. Conversely, only $2.5 \%$ of the proteins that increased in abundance, and $1.5 \%$ of the proteins that decreased in abundance, were found in the down-regulated and up-regulated transcripts, respectively. These numbers substantiate somewhat the iTRAQ results despite the fact that there is not always good agreement between mRNA and protein levels.

The DAPs were classified into the broad GO categories described above (Figure 2.5). As the stigma matures, most categories display more proteins that increase in abundance than decrease in abundance, with the most highly abundant categories being Carbohydrate Metabolic Process, Cell Wall Organization or Biogenesis, Defense, Lipid Metabolic Process, Metabolic Process, Protein Metabolic Process, Regulation of Cellular Process, Response to Stress, Signaling, and Transport. In comparison, more proteins had 


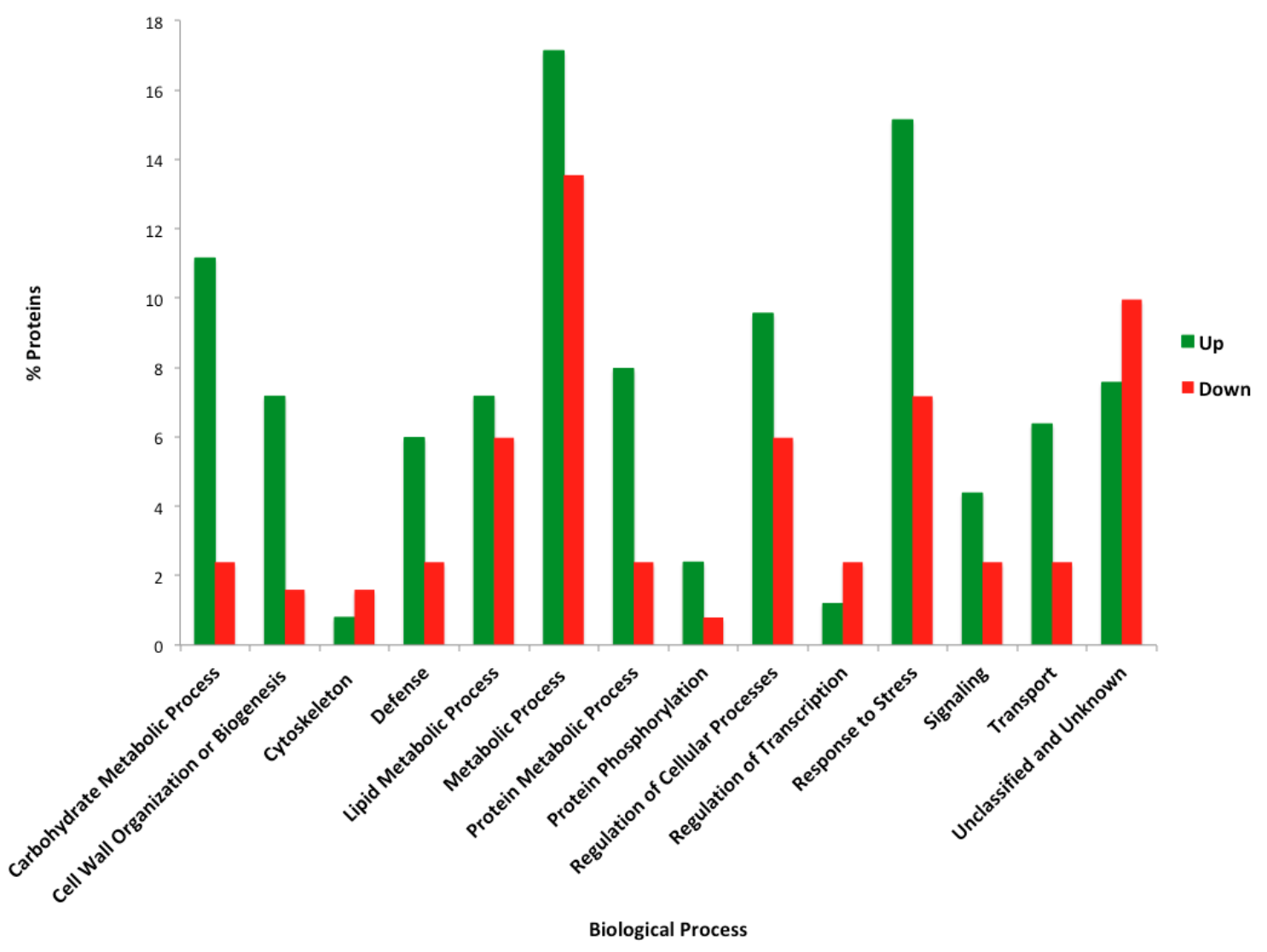

Figure 2.5. Percentage of proteins that increased and decreased in abundance during $B$. carinata stigma maturation. 
decreased protein levels in the categories Cytoskeleton and Regulation of Transcription. Many of the proteins present within these categories are described below and represent good candidates (especially proteins with increased abundance) for further study of the pollen-stigma interactions.

\subsubsection{Pollen-Pistil Interactions}

It is beyond the scope of this chapter to provide a detailed discussion of all the various types of proteins identified within the $B$. carinata mature pollen and stigma proteomes (Tables S2.1-S2.7). Since the proteins identified in this study correspond to the proteins present when the mature pollen and stigma meet, focus will be placed on the roles of some of these proteins in the context of the different phases of pollen-stigma interactions. Although advantage will be taken of the information pertaining to the orthologous Arabidopsis thaliana proteins shown to function in these processes, it will nonetheless become evident that many of the proteins identified in this study still have no known role in the pollen and stigma.

\subsubsection{Pollen Adhesion}

The stigma is the first tissue of the gynoecium to come into contact with the pollen. Binding of the pollen to the stigma shows specificity since interfamilial pollen grains adhere poorly to stigmas (Lord, 2002; Edlund et. al., 2004). In Arabidopsis, the strength at which the pollen adheres to the stigma increases as time progresses. Adhesion does not rely on the pollen coat as evidenced by the ECERIFERUM (CER) mutant cer62, lacking a pollen coat, being still able to adhere to the stigmatic surface. In addition, 
the initial adhesive step may also not be based on protein-protein interactions and most likely depends on biophysical interactions between the stigma surface and the pollen exine (Hiscock and Allen, 2008; Edlund et. al., 2004). The pollen exine is therefore important for proper pollen-stigma adhesion and defects in pollen exine formation have led to sterility (Chapman and Goring, 2010). Even though the mature pollen grain possesses a fully formed exine, at least 20 diverse proteins involved in exine formation were identified within the pollen proteome (Table S2.1) and in most cases it remains unclear whether these serve additional roles upon reaching the stigma. Some of these proteins included Brassica orthologs of Arabidopsis apyrase 7 (APY7), the apy 7 mutant pollen showed an abnormal exine reticulate pattern and the Atapy6Atapy 7 double knockout had severe defects in pollen exine patterning and a deformed pollen shape resulting in reduced male fertility (Yang et. al., 2013); callose synthase 5 (CALS5) which is the main enzyme for callose layer biosynthesis surrounding tetrads, and in a cals 5 mutant, the thickness of the callose wall was greatly reduced and led to aberrant exine patterns (Dong et. al., 2005; Yang et. al., 2013); a membrane-associated protein called DEFECTIVE IN EXINE FORMATION 1 (DEX1) with dex 1 mutants having delayed and significantly reduced primexine deposition leading to the random deposition of sporopollenin on the plasma membrane (Paxson-Sowders et. al. 2001); the NO PRIMEXINE AND PLASMA MEMBRANE UNDULATION (NPU) protein whose mutation caused a defect in callose synthesis leading to the complete absence of primexine deposition and plasma membrane undulation and affected sporopollenin deposition resulting in male sterility (Chang et. al., 2012); and cysteine protease 51 (CP51) whose reduced expression by RNA interference (RNAi) led to aborted 
microspores and a male sterile phenotype due a defective pollen exine (Yang et. al., 2014).

The second stage in adhesion involves protein-protein interactions between the pollen grain and the stigma. Mobilization of the pollen coat forming a "foot" of contact between the pollen grain and the papillar cell (Figure S4) enhances pollen-stigma adhesion by allowing proteins within the pollen coat and on the surface of the stigma to come into contact (Rejón et. al., 2016; Edlund et. al., 2004). In B. rapa, the stigmaspecific secreted glycoprotein $S$-locus-related1 (SLR1) was shown to bind with high affinity to the class A pollen coat proteins SLR1-BP1 and 2, while the pollen coat peptide pollen coat protein A1 (PCP-A1) was found to bind to the stigma wall-anchored $S$-locus glycoprotein (SLG) protein in B. oleracea. Both these interactions are postulated to strengthen pollen-stigma adhesion (Rejón et. al., 2016). SLR1-BP and PCP-A1 were identified in the B. carinata pollen proteome (Table S2.1), while SLR1 and SLG proteins were identified in the stigma (Table S2.1) and shown to increase in abundance as the stigma matured (Table S2.6) as shown previously (Hackett et. al., 1996; Robert et. al., 1994).

Lastly, B. carinata proteins representing all 8 subunits of the exocyst complex were identified in the stigma proteome (Table S2.1). These subunits act as a tether to dock vesicles for fusion to the plasma membrane during regulated or polar secretion (Doucet et. al., 2016). Down-regulation of each member in the Arabidopsis stigma using RNA-silencing led to a reduction in pollen adherence to the stigma (Safavian et. al., 2015). It is possible that the exocyst complex may be involved in the transport of vesicles carrying proteins that facilitate pollen adhesion to the stigmatic surface. 


\subsubsection{Pollen Recognition}

The formation of the "foot" (Figure S4) not only contributes to adhesion, but also brings together stigmatic and pollen coat proteins involved in pollen recognition (Chapman and Goring, 2010). Pollen pre-fertilization barriers control whether or not a particular pollen landing on the stigma will successfully lead to fertilization (Kitashiba and Nasrallah, 2014). Within the Brassicaceae, S-haplotype-specific binding of the small pollen coat protein $S$-locus cysteine rich/S-locus protein 11 (SCR/SP11) to the stigma localized $S$ Receptor Kinase (SRK) triggers a self-incompatibility (SI) response that blocks hydration and germination, leading to the rejection of self-pollen on the surface of the stigma (Rejón et. al., 2016). SP11/SCR was identified in the pollen proteome (Table S2.1), while numerous orthologs to SRK were detected in the stigma (Table S2.1). Since B. carinata exhibits self-compatibility, it is possible that some of the proteins involved in the SI response are not functional since deletion or inactivation of one or both of these genes is believed to underlie the evolutionary switch from an outbreeding to an inbreeding mating system within the Brassicaceae (Kitashiba and Nasrallah, 2014; Nasrallah, 2002).

In addition to the SRK orthologs identified in the stigma proteome, three Sdomain receptor-like kinases (RLKs) were also found in the stigma proteome (Table S2.1), and one S-domain RLK ortholog was identified in the pollen proteome (Table S2.1). Also, four proteins related to the Papaver rhoeas S1 self-incompatibility protein were identified in the pollen proteome (Table S2.1). SI is also controlled by a single multi-allelic $S$-locus in $P$. rhoeas, and homologues of the $\mathrm{S} 1$ self-incompatibility protein were also identified in the self-compatible Arabidopsis spp. (Ride et. al., 1999). The 
precise role of these different proteins within self-compatible species has not yet been elucidated, however it is also possible that some of these proteins could have a role in defense.

\subsubsection{Pollen Hydration}

After compatible pollen recognition, the stigmatic papilla initiates cellular responses in order to facilitate the rehydration of the partially desiccated pollen grain. One of the earliest of these responses is vesicular trafficking. In Arabidopsis, the papillar cell wall was observed to expand at the zone of pollen adhesion 10 minutes postpollination (Kandasamy et. al., 1994). In B. napus, multi-vesicular bodies were observed to fuse to the papilla plasma membrane towards the pollen contact site releasing extracellular vesicles into the cell wall, whereas in Arabidopsis secretory vesicle-like structures fused to the papilla plasma membrane 10 minutes post-pollination (Doucet et. al., 2016). These vesicles/multi-vesicular bodies were postulated to be carrying cargo needed to facilitate water transfer, as well as cargo needed for papillar cell wall expansion, pollen tube penetration and pollen tube growth (Safavian et. al., 2015; Goring et. al., 2017). As previously mentioned, we identified all 8 members of the exocyst complex implicated in membrane trafficking and polar secretion within the stigmatic papillae of Arabidopsis in response to compatible pollination. Knockdown/knockout of the expression of all 8 subunits resulted in a reduction in pollen adhesion, hydration, germination, penetration, pollen tube growth, seed set and overall fertility in Arabidopsis (Safavian et. al., 2015). Additionally, 12 EXO70 orthologs were identified in the $B$. carinata stigma proteome (Table S2.1), including EXO70H7 which increased in 
abundance as the stigma matured (Table S2.6). EXO70 genes are expressed in exocytosis active cells and cell type- and/or cargo-specific exocytotic activities are postulated to be regulated by different EXO70 isoforms (Li. et. al., 2010). With the exception of EXO70A1 (Samuel et. al., 2009), functions of the other EXO70 proteins found within the stigmatic papillae proteome have not yet been elucidated.

Proteins involved in water homeostasis are also important for pollen hydration. The papillar cells of the mature stigma are turgid in order to hydrate pollen and the stigma contains water underneath the waxy cuticle (Rehman and Yun, 2006; Hiscock and Allen, 2008; Gao et. al., 2016). Ions like potassium $\left(\mathrm{K}^{+}\right)$and chloride $\left(\mathrm{Cl}^{-}\right)$are responsible for maintaining the cell's turgidity. For example, in leaves, the movement of water into, and therefore the swelling of guard cells, which like the stigmatic papillae are epidermal cells, is governed by $\mathrm{K}^{+}$ions and anions like $\mathrm{Cl}^{-}$(Rehman and Yun, 2006, Jossier et. al., 2010). Several orthologs of $\mathrm{K}^{+}$channels, transporters and antiporters were identified within the stigma proteome (Table S2.1), one of which, the potassium channel beta subunit $\mathrm{KAB} 1$, increased in abundance as the stigma matured (Table S2.6). The syntaxin of plants homolog, SYP121, shown to facilitate $\mathrm{K}^{+}$uptake by $\mathrm{K}^{+}$channel trafficking and recycling to the plasma membranes in leaves during stomatal re-opening (Eisenach et. al., 2012) was also detected. In addition, 6 stigma chloride channel orthologs were identified such as CLCc shown to regulate stomatal opening by regulating chloride ion homeostasis (Jossier et. al., 2010), and 4 mechanosensitive ion channels including the mechanosensitive channel of small conductance-like 7 (MSL7) that increased in abundance in the mature stigma (Table S2.6). Mechanosensitive ion channels regulate osmotic forces by conducting ions and other osmolytes in response to 
increased membrane tension (Hamilton and Haswell, 2017). It is possible that the swelling of the cell membrane and increased membrane tension in the turgid stigma papillae causes the mechanosensitive ion channels to open, releasing ions and other small osmolytes thus causing the water potential inside the cell to increase. This would in turn cause water to exit the stigmatic papillae, possibly through aquaporins, which are membrane proteins that regulate the flow of water across cellular membranes (Soto et. al., 2008). Several B. carinata aquaporins were identified (Table S2.1) with two plasma membrane intrinsic proteins PIP2F and PIP2B increasing in abundance during stigma maturation (Table S2.6).

The pollen grain, in turn, needs to take up the water provided by the papilla. Three aquaporins were identified in the pollen proteome (Table S2.1), as well as SYP61 known to coordinate the trafficking of the plasma membrane aquaporin PIP2;7 (Hachez et. al., 2014). Pollen hydration is known to be facilitated by the lipid-rich pollen coat and it has been postulated that its constituent tapetum-derived oleosins and extracellular lipases (EXLs) act synergistically to modify lipid composition at the pollen-stigma surface to facilitate hydration (Rejón et. al., 2016). Orthologs of two tapetal oleosins, EXL4, and EXL6 were part of the pollen proteome (Table S2.1).

Desiccated pollen cell membranes are rigid and less able to accommodate hypoosmotic swelling. The rehydration process can therefore damage cell membranes and compromise pollen viability. As a result, hydration needs to be properly regulated. Ion channels are therefore important to maintaining plasma membrane integrity during pollen hydration by regulating turgor pressure. Ion channels are also a necessity during pollen germination and tube growth to ensure that the tube is turgid enough to break through the 
pollen cell wall, but also to ensure that the pollen tube does not rupture prematurely (Hamilton and Haswell, 2017; Li et. al., 2017). Seventy-seven orthologs of ion channels were identified within the pollen proteome (Table S2.1) including two shaker pollen inward $\mathrm{K}^{+}$channel (SPIK) important to pollen hydration (Li et. al., 2017), two MSL7 homologs, one piezo-type mechanosensitive ion channel, chloride channels CLCc and CLCf, as well as 8 cation/proton exchanger (CHX) postulated to allow osmotic adjustments and $\mathrm{K}^{+}$homeostasis during pollen rehydration and germination (Sze et. al., 2004).

\subsubsection{Pollen Germination}

For the purposes of this discussion, germination is considered to encompass the events involved in breaching the pollen cell wall. After hydration, the pollen grain establishes an internal polarity relative to external signals in order for the tube to breach the pollen cell wall from a single point and then grow to reach the stigmatic surface (Edlund et. al., 2004). The pecto-cellulosic intine wall layer of the pollen grain contains two distinct layers; the exintine and the endintine. The endintine is the precursor for the pollen tube wall while the exintine harbors channels containing enzymes (Blackmore and Knox, 1990). In the Brassicaceae, the pollen tube needs to dissolve the exintine layer before emerging through either the aperture or the interaperture exine wall (Edlund et. al., 2004). Eighty-three enzymes involved in cell wall degradation were identified within the pollen proteome, some of which may facilitate degradation of the exintine layer of the pollen grain (Table S2.1). Several of these proteins may also help to digest the cell wall of the stigma and transmitting tract to facilitate pollen tube penetration (see below). 
Additionally, a UDP-glycosyltransferase family protein called "bursting pollen” (BUP) involved in the correct orientation of the germination plaque (deposition of callose near the pollen wall at the site of contact with the stigma) and the pollen tube tip (Hoedemaekers et. al., 2015) was present within the pollen proteome.

It has been postulated that the source of water during hydration may provide directional cues that establish polarity (Edlund et. al., 2004), but it is quite possible that calcium signaling from the stigma to the pollen grain also plays a role. Calcium influx at the pollen tube membrane tip from external sources contributes towards pollen tube guidance, and a cytosolic increase in calcium within the apical region of $A$. thaliana stigmatic papillae was observed immediately prior to pollen germination (Steinhorst and Kudla, 2013; Chapman and Goring, 2010; Cheung and Wu, 2008). Fifteen B. carinata calcium $\left(\mathrm{Ca}^{2+}\right)$ transporters were identified within the stigma proteome (Table S2.1) and one, the cyclic nucleotide-gated ion channel 1 (CNGC1), was found to increase in abundance in the mature stigma (Table S2.6). Similarly, the pollen proteome possessed $21 \mathrm{Ca}^{2+}$ transporters (Table S2.1) including the plasma membrane localized autoinhibited $\mathrm{Ca}^{2+}$ ATPase 9 (ACA9) whose mutant was found to result in partial male sterility (Schiøtt et. al., 2004).

Reactive oxygen species (ROS) have been identified as important signaling molecules in pollen germination and pollen tube growth. In addition, respiratory burst oxidase homologues (RBOHs) activated via $\mathrm{Ca}^{2+}$, lead to hydrogen peroxide $\left(\mathrm{H}_{2} \mathrm{O}_{2}\right)$ mediated activation of $\mathrm{Ca}^{2+}$ channels increasing the cytosolic $\mathrm{Ca}^{2+}$ levels (Steinhorst and Kudla, 2013). ROS was required for pollen germination in lily (Smirnova et. al., 2009), kiwifruit (Speranza et. al., 2012), tobacco, and olive (Potocký et. al., 2012), and locally 
restricted ROS production at a pollen grain aperture was required for outgrowth at that particular aperture. ROS has also been implicated in pollen exine breach in a two-step process where a swollen intine (due to turgor pressure from hydration) pushes against an exine weakened by redox reactions present at the pollen-stigma interface, resulting in exine rupture at the site of pollen tube emergence (Edlund et. al., 2017). Eighty-four proteins involved in ROS metabolism were identified in the stigma and pollen proteomes (Table S2.1). For example, within the pollen proteome, B. carinata orthologs of 2 Arabidopsis RBOH's, 16 peroxidases, 2 catalases, and 6 superoxide dismutases were identified, whereas the stigma proteome contained orthologs to a $\mathrm{RBOH}, 2$ catalases, 18 peroxidases, and 8 superoxide dismutases (Table S2.1). One superoxide dismutase (SODB) and a glutathione peroxidase (GPX7) were found to increase in abundance as the stigma matured (Table S2.6). Interestingly, orthologs of peroxidase (PRX42) and a superoxide dismutase (SODCP) had decreased protein levels in the mature stigma (Table S2.7). Protein isoforms with increased abundance are most likely responsible for the initial stages of pollination and could be investigated for their role in pollen germination, whereas isoforms with a decreased abundance could possibly have been involved in earlier stigma developmental processes.

\subsubsection{Pollen Tube Growth}

Once the pollen tube has successfully emerged from the grain, it grows through the foot to penetrate the stigmatic pellicle and waxy cuticle. Initial invasion of the pollen tube through the stigma requires enzymatic modification of these layers. The proteinaceous pellicle of dry stigmas was proven to contain enzymes that are postulated 
to be involved in pollen tube breach and removal of the pellicle prevents compatible pollen tubes from entering the stigma (Chapman and Goring 2010; Heslop-Harrison and Heslop-Harrison, 1975; Hiscock et. al., 2002). The B. carinata stigma contained 176 proteases (Table S2.1), 7 of which increased in abundance as the stigma matured (Table S2.6) and could potentially represent candidates for the unknown proteases that facilitate pollen tube invasion of the stigma. Numerous proteases were also identified within the mature pollen proteome (Table S2.1).

The mature stigma also contained 18 esterases (Table S2.1) and the three enzymes that increased in abundance late in development (Table S2.6) might be accumulating in preparation for pollen tube penetration of the cuticle. Esterases known as cutinases are important for breaking down the stigma cuticle as treatment of the stigmas with serine esterase inhibitors blocked pollen tube penetration (Edlund et. al., 2004). However, no cutinases were identified within the mature $B$. carinata mature stigma or pollen proteomes. A GDSL lipase primarily expressed in the pollen that fits the description of a plant cutinase has been identified in A. thaliana (Takahashi et. al., 2010) and several GDSL lipases were identified within the proteomes of both the mature stigma and pollen (Table S2.1) and some could potentially serve the role of a cutinase.

Within the Brassica ssp., the pollen tube then grows between the inner and outer layers of the papillar cell wall before reaching the base of the stigma and entering the style transmitting tract. Invasion through these layers requires various cell wall modifying enzymes involved in the degradation and remodeling of pectin, cellulose and hemicellulose (Chapman and Goring 2010). Numerous enzymes involved in cell wall degradation and modification were identified within the stigma proteome (Table S2.1), 
17 of which had elevated protein levels in the mature stigma and include an endo-1,3(4)$\beta$-D-glucanase-like protein, a $\beta$-D-xylosidase, a $\beta$-galactosidase, pectate lyases, polygalacturonases, a $\beta$-glucosidase, a xyloglucan endotransglucosylase/hydrolase, pectin acetylesterases, and several pectin methylesterases (PMEs) (Table S2.6). In addition, 9 expansins, known to be involved in cell wall loosening and expansion (Mollet et. al., 2013), were detected within the stigma proteome with two that increased in abundance as the stigma matured (Tables S2.1 and S2.6), while four were found in the pollen proteome (Table S2.1). Furthermore, several extensins, thought to be involved in cell wall extension (Lora et. al., 2016), were identified within both the mature pollen and stigma proteomes (Table S2.1).

Initial pollen tube growth is autotrophic and uses stored nutrients within the pollen grain. However, subsequent growth is heterotrophic and requires assistance from pistil tissues (Yue et. al., 2014). A massive deposition and remodeling of cell wall polysaccharides is required to promote fast pollen tube elongation and proteins involved in the biosynthesis of cell wall polysaccharides are involved in this process (Mollet et. al., 2013). As mentioned earlier, Nucleobase-containing Compound Metabolism contained the most number of enriched sub-categories in both the pollen and stigma proteomes, and 156 proteins involved in the synthesis and metabolism of UDP-sugars, precursors for the synthesis of cell wall polysaccharides, were identified. In addition, 107 proteins contributing to cell wall biogenesis including enzymes involved in cellulose, pectin, and hemicellulose biosynthesis were found in both the mature stigma and pollen (Table S2.1). 
Pollen tube walls contain two layers at the shank of the pollen tube; an outer/primary wall that is mainly consisted of pectin, cellulose and hemicellulose, and an inner/secondary wall consisting of cellulose and callose (Hoedemaekers et. al., 2015; Li et. al., 2012). Pollen tubes grow exclusively at the tips and the wall at the tip consists of a single layer of mostly pectin. The pectin synthesized within the Golgi apparatus is methyl-esterified and transported via vesicular trafficking to the tip. As the tip extends, the pectin is de-esterified by the action of PME and ends up in the more distal region of the outer wall where the exposed pectin carboxyl groups cross-link to $\mathrm{Ca}^{2+}$ which causes rigidification of the cell wall (Bosch and Hepler, 2005; Mollet et. al., 2013).

VANGUARD1 (VGD1) whose ortholog is found in the $B$. carinata pollen is an example of such a PME whose mutation retards pollen tube growth (Jiang et. al., 2005). Twenty eight $B$. carinata orthologs of Arabidopsis PMEs were identified within the pollen proteome (Table S2.1). Similarly, 101 other pollen proteins shown to be involved in pectin biosynthesis and metabolism were identified, along with 51 proteins involved in cellulose and hemicellulose synthesis and metabolism (Table S2.1).

During pollen tube growth, callose is deposited at periodic intervals behind the growing tip to form plugs that maintain a region of concentrated cytoplasm containing organelles, the vegetative nucleus, and the two sperm cells in the apical expanding region, while simultaneously separating the viable from degenerating region of the tube (Mollet et. al., 2013). Thirteen callose synthases, as well as 11 beta-glucanases and 46 beta-glucosidases were also identified within both the mature stigma and pollen (Table S2.1) proteomes with some increasing in abundance as the stigma matured (Table S2.6). 
Pollen tubes grow at their tips through a polarized growth process and a tipfocused $\mathrm{Ca}^{2+}$ gradient is required for pollen germination and tube growth (Cheung and Wu, 2008; Steinhorst and Kudla, 2013). Calcium is involved in a variety of processes required for germination and tube growth including cell wall rigidity at the distal regions of pollen tube via cross linking with de-esterified pectin, cytoskeleton organization via the regulation of actin-binding proteins (ABPs), and vesicular trafficking (Cheung and Wu, 2008; Bosch and Hepler, 2005). Calcium can also trigger enhanced ROS production by RBOHs via calcium-dependent protein kinases like CPK26 (Gilroy et. al., 2014) whose ortholog was identified within the pollen proteome (Table S2.1). CPKs function in multiple signal transduction pathways downstream of cytosolic $\mathrm{Ca}^{2+}$ elevation and the CPK orthologs identified within the pollen proteome may control a variety of cellular processes ranging from the regulation of ion channels to hormone signaling. A total of 9 pollen B. carinata CPKs were identified, in fact, nearly $150 \mathrm{PKs}$ were identified in the pollen proteome (Table S2.1) and although many have known roles in pollen development and pollen tube growth whose description is beyond the scope of this discussion, most still have undefined roles in that context.

In addition to a tip focused $\mathrm{Ca}^{2+}$ gradient, an acidic tip and an alkaline subapical cytoplasm are essential for growth polarity by regulating ABPs (Cheung and $\mathrm{Wu}, 2008$ ). Hydrogen distribution is maintained by an $\mathrm{H}^{+}$influx at the apex through low affinity nonselective cationic channels and an efflux along the subapical membrane through localized $\mathrm{H}^{+}$-ATPases that are excluded from the tip (Boavida et. al., 2005; Song et.al., 2009). Several proton transporters were identified within the mature pollen (Table S2.1) and hydrogen transport was an enriched sub-category within the mature pollen proteome 
(Table S2.5). The 8 CHXs mentioned earlier and the 14 P-type, autoinhibited $\mathrm{H}^{+}$-ATPase (AHA) proton pumps, 26 vacuolar $\mathrm{H}^{+}$-ATPase (VHA) subunits, and $6 \mathrm{~V}$-type pyrophosphate-energized membrane protein pumps (VHPs) could contribute to $\mathrm{H}^{+}$influx at the pollen tube apex and/or to subapical $\mathrm{H}^{+}$efflux.

Phosphoinositides (PIs) represent another important class of signaling molecules during pollen germination and tube growth. PIs regulate cytoskeletal dynamics by regulating $\mathrm{ABPs}$, ion channel activity and vesicular trafficking, and therefore impact the delivery of cell wall material and modifying enzymes to the growing polar tip (Cheung and Wu, 2008; Steinhorst and Kudla, 2013). Phospholipase D (PLD) hydrolyzes phosphatidylcholine to produce phosphatidic acid (PA). Capping proteins (CPs) that bind and modulate actin polymerization are inhibited by PA and phosphatidylinositol 4,5biphosphate (PIP2) (Cheung and Wu, 2008; Jimenez-Lopez et. al., 2014). Three CPs and 8 PLDs were identified within the B. carinata pollen proteome (Table S2.1). Phospholipase C (PLC) hydrolyzes PIP2 to inositol 1,4,5-triphosphate (IP3) and diacylglycerol (DAG). PIP2 regulates actin depolymerization factors (ADFs) which bind to actin filaments stimulating their depolymerization, IP3 may induce $\mathrm{Ca}^{2+}$ increase, and DAG may serve as a precursor for PA (Cheung and Wu, 2008; Steinhorst and Kudla, 2013). Four Arabidopsis PLCs and four PLC-like proteins were identified within the pollen proteome. In addition, three pollen ADFs were found (Table S2.1).

Well-regulated phosphatidylinositol-4-phosphate, PIP2, and phosphatidylinositol 3,5-biphosphate (PtdIns $(3,5) \mathrm{P}_{2}$ ) pools are necessary for polarized secretion in plants. Phosphoinositide (PI) phosphatases and kinases contribute to the regulation of these PI pools (Chapman and Goring, 2011; Serrazina et. al., 2014). B. carinata orthologs of 8 
Arabidopsis PI phosphatases and 8 PI kinases were identified within the pollen proteome (Table S2.1). Mutations in these enzymes can affect PI pools and may lead to pollen lethality, for example, the double mutant of the orthologous 1-phosphatidylinositol-3phosphate 5-kinase proteins FORMS APLOID AND BINUCLEATE CELLS (fab) lafablb exhibited pollen lethality as a consequence of a failure in vacuole rearrangement (Serrazina et. al., 2014).

Rac/Rop GTPases regulate multiple linear as well as intersecting signaling pathways. They control the phosphorylation and therefore inhibition of ADF and can increase intracellular $\mathrm{Ca}^{2+}$ in the apex via interaction with ROP-interactive CRIB motifcontaining protein 3 (RIC3) (Cheung and Wu, 2008). Pollen RIC3 as well as 6 pollen Rac/Rop GTPases were identified (Table S2.1). Eight regulators of Rac/Rop GTPases were also identified within the pollen proteome including two orthologs of RHO GTPaseactivating protein ROP ENHANCER 1 (REN1) which restricts active ROP1 to the pollen apical cap (Hwang et. al., 2008), four ROP guanine nucleotide exchange factors (ROPGEFs) which activate Rac/Rop GTPases, and a RHO GDP-dissociation inhibitor 1 (RHOGDI1) which inactivates Rac/Rop GTPases (Cheung and Wu, 2008) (Table S2.1). Cytoskeleton Organization was also among the enriched sub-categories in the $B$. carinata pollen (Table S2.5). In the pollen tube, the role of actin filaments is related to the transport of organelles and vesicles, and $\mathrm{ABPs}$ and $\mathrm{ABP}-$ related proteins regulate this process by controlling actin polymerization/depolymerization (Cai et. al., 2015; Steinhorst and Kudla, 2013). Multiple pollen-expressed ABPs and ABP-related proteins were identified within the pollen proteome, along with several actins, myosins, profilins and villins that have been demonstrated to play roles in pollen tube growth (Table S2.1). 
The growing tip of the pollen tube requires vesicle-mediated transport, which is essential for the deposition of cell membrane and wall materials, as well as proteins involved in cell wall modification at a restricted tip area of the plasma membrane (Tan et. al., 2016; Mollet et. al., 2013). The tip region consists of a clear zone composed of numerous Golgi-derived vesicles that migrate toward the apex in the cell cortex and accumulate at the apex forming an inverted cone-shaped area where they fuse with the plasma membrane to sustain pollen tube growth (Mollet et. al., 2013). Accordingly, Transport was one of the categories with the most number of enriched sub-categories in the pollen proteome (Figure 2.3) with 45 out of the 53 transport sub-categories being involved in vesicular trafficking and/or localization (Table S2.5). Some of these enriched processes, namely Vesicle-Mediated Transport, Golgi Vesicle Transport, Vesicle Fusion, Membrane Fusion, Exocytosis, Retrograde Transport, and some of their constituent proteins will be discussed in greater detail below. Due to the extensiveness of the pollen transport category, the discussion will focus primarily on examples of orthologs of known Arabidopsis players involved in vesicular transport.

For example, RAB GTPases regulate vesicle budding and targeting specificity, and mediate vesicle fusion, and are therefore important for vesicular trafficking during pollen germination and tube growth. Thirty one B. carinata RAB GTPases were identified in the pollen proteome with many having a demonstrated role in pollen tube growth such as RABA4D (Szumlanski and Nielsen, 2009), as well as RABD2B and RABD2C (Peng et. al., 2011), and nearly 50\% are shared with the stigma (Table S2.1). RAB GTPases are inactivated by RAB guanine nucleotide dissociation inhibitors (RABGDIs) and RAB GTPase-activating proteins (RABGAPs) (Cheung and $\mathrm{Wu}, 2008$ ). 
Orthologs of three Arabidopsis RABGDI and 7 RABGAPs were identified in the pollen proteome (Table S2.1).

A GTP-binding protein called ADP-ribosylation factor (ARF) is involved in vesicle coating and vesicular budding. ARF is activated by ADP-ribosylation factor guanine-nucleotide exchange factor (ARFGEF) and can be inhibited by ADP-ribosylation factor GTPase-activating protein (ARFGAP) (Min et. al., 2013; East and Kahn, 2011). Within the pollen proteome, orthologs of 3 Arabidopsis ARFs, 12 ARFGEFs, and 19 ARFGAPs were identified and many still do not have well defined roles in the pollen. In addition, multiple pollen SEC proteins, which are members of the exocyst complex involved in protein transport, were identified (Table S2.1) with many, such as SEC3, SEC5, SEC6, SEC8, or SEC15A, playing a role in pollen germination and tube growth (Cole et. al., 2005; Hála et. al., 2008).

SNARE proteins play a role in controlling membrane fusion (Sansebastiano et. al., 2014). For example, syntaxin of plants 124 (SYP124) was shown to be concentrated in the apical and subapical regions of the elongating pollen tube and is postulated to form SNARE complexes with vesicle-associated membrane protein 725 (VAMP725) and VAMP726 during pollen tube tip growth (Silva et. al., 2010; Ichikawa et. al., 2015; Slane et. al., 2017). Multiple B. carinata orthologs of Arabidopsis SYP, SNARE and VAMP proteins were identified in the pollen proteome (Table S2.1).

The massive amount of exocytosis occurring at the pollen tube tip is believed to exceed the requirements for maintaining tube growth rates. Therefore, endocytosis and vesicular recycling via retrograde transport is critical for polarized pollen tube growth. Orthologs of the conserved oligomeric Golgi complex subunits 3 and 8 (COG3 and 
COG8) essential for pollen tube growth (Tan et. al. 2016) were detected within the pollen proteome and are components of a COG complex that is postulated to be involved in the tethering of retrograde intra Golgi vesicles. The formation of transport vesicles from the donor organelle is accomplished by snipping the neck of the invaginated membrane. This is accomplished by dynamin-related proteins (Fujimoto and Tsutsumi, 2014) and 9 dynamin-like proteins likely involved in endocytosis were identified including DRP2A and DRP2B (Table S2.1). Secretory carrier membrane proteins (SCAMPs) are type IV membrane proteins that are localized to the plasma membrane and early endosome or trans-Golgi network in the plant endocytic pathway. Vacuolar sorting receptors (VSRs) are type I integral membrane proteins that mediate biosynthetic protein traffic in the secretory pathway to the vacuole (Wang et. al., 2010). Three B. carinata pollen SCAMPs and one VSR were identified (Table S2.1). Additionally, 43 vacuolar protein sorting-associated proteins (VPSs) were found including orthologs of Arabidopsis VPS 16 (Hicks et. al., 2004), VPS 41 (Hao et. al., 2016), VPS 52A (Lobstein et. al., 2004), and VPS 53 (Wang et. al., 2008) (Table S2.1), which are implicated in the endocytic pathway and have demonstrated roles in pollen tube growth. Very little is known about the potential role of the other identified VPSs in this process.

Many of the proteins discussed above are pollen proteins with a demonstrated role in pollen tube growth, but in many cases many of the same or related proteins are found in the stigma. However, information is scarce regarding the role of these stigma proteins in pollen tube growth and more research is required. 


\subsubsection{Pollen Tube Guidance}

Growth of the pollen grain through the pistil also requires cell-cell adhesion (Mollet et. al., 2013). Many proteins including several transmembrane 9 and Ofucosyltransferase proteins postulated to be involved in cell adhesion (Benghezal et. al., 2003; Verger et. al., 2016), as well as orthologs of GPI-anchored adhesion-like proteins shown to be involved in cell-cell adhesion in yeast (Dranginis et. al., 2007) were identified within the mature stigma and/or pollen proteomes (Tables S2.1). Proteins involved in adhesion are proposed to also be involved in pollen tube guidance. Lily pollen tubes were shown to adhere to the inner surface of the transmitting tract in vivo and grow using an adhesion-mediated pollen tube guidance mechanism (Dresselhaus et. al., 2013). Arabinogalactan proteins are believed to be candidate molecules for pollenpistil adhesion and were also shown to both stimulate pollen tube growth in vitro and act as a chemo-attractant (Rejón et. al., 2016; Dresselhaus et. al., 2013). Lipid transfer proteins (LTPs) shown to interact with pectins play an important role in the formation of an adhesive matrix needed for pollen tube guidance (Dresselhaus et. al., 2013; Mollet et. al., 2013). The arabinogalactan proteins and lipid transfer proteins identified within the mature stigma and pollen proteomes could be contenders for such roles (Tables S2.1).

Chemo-attractant gradients in the pistil are important to pollen tube guidance. Gamma-amino butyric acid (GABA) present as a gradient starting from the stigma and increasing in concentration towards the ovule may serve to guide the pollen tube in the pistil. The A. thaliana pollen-pistil 2 (pop2) mutant lacking a functional GABA transaminase in both the pollen and pistil displayed abnormal pollen tube guidance (Wilhelmi and Preuss 1996). Two GABA transaminase orthologs were identified in the 
mature stigma proteome, whereas one was identified within the pollen proteome (Table S2.1). An ortholog of a plantacyanin protein that forms a similar concentration gradient along the pistil (Dong et. al., 2005) was also identified in the mature stigma proteome (Table S2.1).

\subsection{Conclusion}

Although the emphasis in this study was placed on pollen-stigma interactions, the comprehensive mature pollen and stigma proteomes obtained not only provide an improved inventory of proteins expressed in these cells, many for the first time, but also point to protein candidates that might deserve further investigation. This is particularly true of the stigma for which little information is available. For example, this analysis identified $3 \mathrm{X}$ as many proteins as the previous Brassica stigma proteome analysis (Nazemof et. al., 2016) and yet revealed more than $12 \mathrm{X}$ and $5 \mathrm{X}$ as many stigma transcription factors (TFs) and protein kinases (PKs) respectively; possibly due to the fact that TFs and PKs are generally low in abundance and are therefore more likely to be missed by a gel-based proteomics approach that relies on protein to be abundant enough to be detected on a gel (Wissing et. al., 2007; Grobei et. al., 2009). Of the 182 TFs identified (Table S2.1), only a few have been previously implicated in gynoecium and/or stigma development including members of the $\mathrm{B} 3$ domain TFs such as the auxin response factor 6 (ARF6) and ARF8 (Wu et. al., 2006), and NGATHA1 (NGA1), NGA2 and NGA3 (Alvarez et. al., 2009; Trigueros et. al., 2009; Martinez-Fernandez et. al., 2014), and the basic helix-loop-helix (bHLH) TFs such as HALF FILLED (HAF), HECATE (HEC) and SPATULA (SPT) (Crawford and Yanofsky, 2011; Moubayidin and 
Østergaard, 2014). Similarly, 231 PKs were identified (Table S2.1) and very few have been shown to be involved in gynoecium and/or stigma development such as the cyclindependent kinases CDKC2 (Cui et. al., 2007) and CDKE1 (Wang and Chen, 2004), as well as the SNF1-related kinase SNRK1.1 (Tsai and Gazzarrini, 2012). Numerous proteins identified in the mature stigma were reported to play roles in epidermal cell development (including root hairs, trichomes, and stomata) which is in line with the fact that stigma papillar cells are also epidermal cells (Lolle and Pruitt, 1999). For example, several of the stigma PKs have been shown to be involved in epidermal cell development including calcium-dependent CPK3 (Wu et. al., 2013), CDKA1 (Borowska-Wykręt et. al., 2013), as well as the transmembrane kinases TMK1 to TMK4 (Xu et. al., 2014) to name but a few, and could be good candidates for the study of their involvement in stigma development. Lastly, this analysis confirmed the presence within the stigma of over 81 proteins involved in photomorphogenesis, circadian rhythm, and flowering for example, and it would be interesting to understand the role of these processes in the stigma. Likewise, $183 \mathrm{PKs}$ and TFs were identified in the mature pollen grain and while some had known roles, 34 still remain to be investigated. 


\section{Chapter 3}

\section{The Analysis of the Triticale (x Triticosecale Wittmack)}

\section{Mature Stigma and Pollen Proteomes}

\subsection{Introduction}

Triticale (x Triticosecale Wittmack ssp.) is a man-made cereal crop belonging to the Triticeae tribe and the Poaceae family (grass family) (Golovnina et. al., 2007;

Szewińska et. al., 2012). Triticale was generated from the interspecific hybridization of durum wheat (Triticum turgidum ssp. durum) as the female parent, and rye (Secale cereale) as the male parent, and exhibits superior agronomical characteristics. It has many of the nutritional qualities of wheat while also having the vigor of rye (i.e. more productive under abiotic stress conditions and less susceptible to diseases than wheat). Primarily, triticale is used for animal feed, but small amounts enter the human food chain (Hills et. al., 2007; Chapman et. al., 2005). Due to the fact that triticale is not generally used in products designed for human consumption and is competitive with other cereals with respect to grain yields, starch content, and price, it could be genetically modified to produce various biopolymers, adhesives, industrial enzymes, and high strength fibers. It has also become a viable platform for bioethanol production (Hills et. al., 2007; Chapman et. al., 2005).

In angiosperms, pollen that is wind-dispersed, as observed in the Poaceae (Friedman and Barrett, 2009), or disseminated via pollinators, as in the Brassicaceae (Kudo, 2003), lands on and adheres to the female stigma. After adhesion and recognition, water flows from the stigma to the partially desiccated pollen grain which 
becomes metabolically active. The pollen then germinates on the surface of the stigma and a pollen tube emerges. The tube then breaches the stigmatic cuticle, invades the intercellular space, and grows in the transmitting tract of the style until it reaches the ovary where it enters the ovule and embryo sac delivering the two sperm cells for double fertilization (Mauseth, 1998; Lord and Russell, 2002). Therefore, in these plants, reproduction is initiated when two cells, the pollen vegetative cell and the stigma papillar cell are brought together from different parts of the plant. When they meet, these cells are both at a late stage of maturation and would be expected to have garnered the necessary protein complement required for their impending interaction. Uncovering the triticale proteins present at this mature stage is thus paramount to investigate the molecular mechanisms underlying the pollen-stigma interaction and will further our understanding in other crop members of the Triticeae such as wheat, barley and rye.

Despite the importance of the pollen and stigma to reproduction, their proteomes have not been extensively investigated in the Poaceae and even less so in the Triticeae. In particular, there have been very few studies of the stigma. To our knowledge, the only studies to date are the gel-based proteomics analyses performed by Sang et. al. (2012) that identified 67 maize stigma-specific/preferential proteins and by Nazemof et. al. (2014) that identified 2184 triticale proteins. With respect to the pollen, Dai et. al. (2007) and Zhu et. al. (2011) used gel-based approaches to study differentially abundant proteins between mature and germinating pollen, and identified 186 rice and 26 maize proteins, respectively. A global proteome analysis also identified 322 rice pollen proteins using gel-based approaches (Dai et. al. 2006). The only gel-free proteomics analysis of mature pollen performed in the Poaceae was with maize and identified 6750 proteins (Chao et. 
al., 2016). To our knowledge, gel-free proteomic approaches have not yet been applied to the characterization of Triticeae mature stigma and pollen proteomes.

In this study, gel-free shotgun proteomics was used to identify 11533 and 2977 triticale mature stigma and pollen proteins, respectively. Furthermore, a differential proteomic analysis of the developing stigma revealed 647 proteins whose abundance differed as the stigma matures in preparation for pollination. This study therefore substantially expands the coverage of the global proteomes of both stigma and pollen within the Triticeae.

\subsection{Materials and Methods}

\subsubsection{Plant Material}

Three biological replicates of the hexaploid triticale (x Triticosecale Wittmack) spring cultivar AC Alta were grown in cabinets under constant humidity of 70\%, 16 hour (h) day and an $8 \mathrm{~h}$ night period, at temperatures of $20^{\circ} \mathrm{C}$ (day) and $18^{\circ} \mathrm{C}$ (night). The plants were watered twice daily and fertilized biweekly with 20-20-20 (NPK).

\subsubsection{Tissue Collection and Microscopy}

Triticale stigmas and anthers were measured in order to identify the lengths corresponding to the bicellular pollen (BCP) and tricellular pollen (TCP) developmental stages. To identify these stages, developing anthers were harvested and fixed in 3:1 70\% ethanol/acetic acid for at least $30 \mathrm{~min}$, washed in 70\% ethanol for $5 \mathrm{~min}$ and Milli-Q water for another $5 \mathrm{~min}$, and stained with 4',6-diamidino-2-phenylindole (DAPI) to a final concentration of $4 \mu \mathrm{g} \mathrm{ml}^{-1}$. The pollen nuclei (Figure 3.1b) were then visualized 
(a)

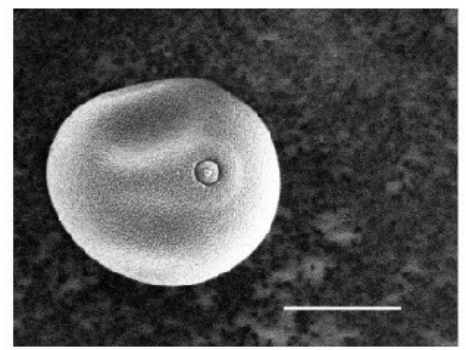

(b)

BCP

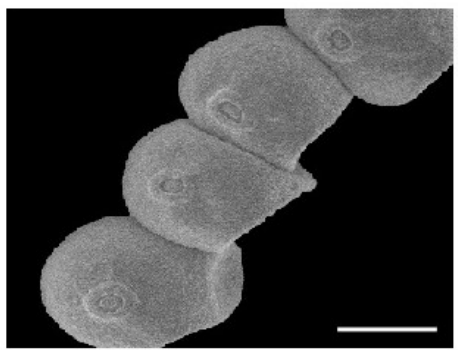

TCP
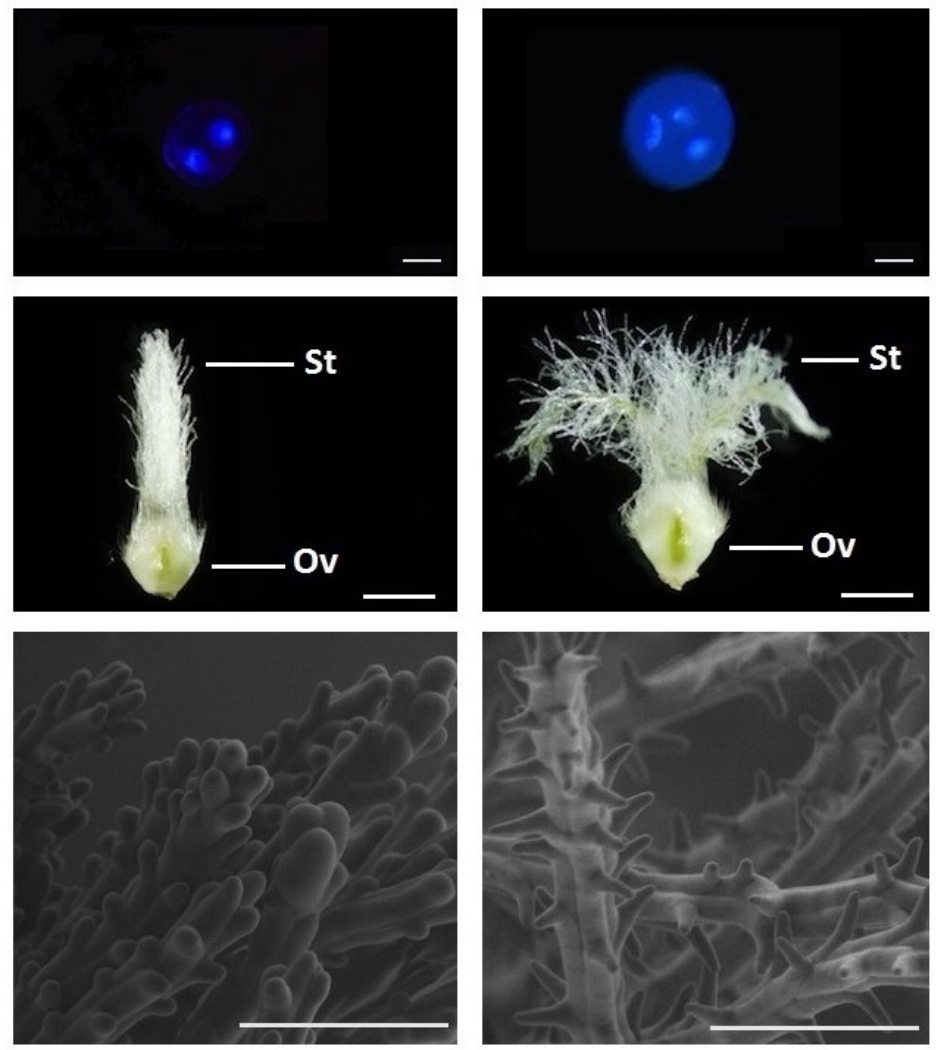

Figure 3.1. Microscopy of mature triticale pollen and stigma at the bicellular pollen (BCP) and tricellular pollen (TCP) stages of development. (a) Scanning electron micrographs of mature pollen. (b) Top row: 4',6-diamidino-2-phenylindole (DAPI)stained pollen. Middle row: Light micrographs of the developing of the pistil tissues. St, Stigma; Ov, Ovary. Bottom row: Scanning electron micrographs of stigma papillae. Light micrographs were taken by Frances Tran, and scanning electron micrographs were taken by Elizabeth Routly. Scale bars: (a) $25 \mu \mathrm{m}$; (b) top row $=20 \mu \mathrm{m}$, middle row from left to right $=0.8 \mathrm{~mm}, 1 \mathrm{~mm}$; bottom row $=100 \mu \mathrm{m}$. 
using a Zeiss axioplan 2 fluorescence microscope (Carl Zeiss Microscopy, Jena, Germany) and images were taken with a Zeiss AxioCam digital camera (Carl Zeiss Microimaging, Jena, Germany), and the Axiovision software (Carl Zeiss Microimaging). Pollen at the BCP stage (Figure 3.1b) was found in florets with green anthers between 4.9-5.5 mm, whereas pollen at the TCP stage (Figure 3.1b) occurred in yellow anthers longer than $5.5 \mathrm{~mm}$.

In this study, the stigma refers to both the stigma and the style due to these structures being merged in triticale. Stigmas at both developmental stages were visualized using a Zeiss Discovery V12 light microscope (Carl Zeiss Microscopy). Stigma tissues and mature pollen grain were also visualized using a Quanta 600 ESEM scanning electron microscope (Thermo Fisher Scientific, San Jose, CA, USA) under environmental conditions, using a low vacuum mode and a gaseous analytical detector (GAD). Stigmas corresponding to the BCP stage were harvested from tillers that contained newly emerging spikes, but with the peduncle still contained within the leaf sheath. At the BCP stage, stigmas measured between $2.8 \mathrm{~mm}$ and $3.4 \mathrm{~mm}$ (Figure $3.1 \mathrm{~b}$ ) and were defined as "early". Bifurcated stigma tissues (longer than $3.4 \mathrm{~mm}$ ) corresponding to the TCP stage were defined as "late" (Figure 3.1b). Mature stigmas and pollen grains were harvested from florets that contained yellow dehiscing anthers (longer than $5.5 \mathrm{~mm}$ ). Harvested tissues were individually inspected under a Leica Zoom 2000 dissecting microscope (Leica Microsystems, Wetzlar, Germany) for the presence of contaminating tissue before being flash frozen in liquid nitrogen and stored at $-80^{\circ} \mathrm{C}$. 


\subsubsection{Trichloroacetic Acid (TCA) Direct Protein Precipitation}

Frozen plant material (approximately 25 and $150 \mathrm{mg}$ of early and late stigma tissues respectively, and $50 \mathrm{mg}$ of pollen) were ground and suspended in $0.25-1 \mathrm{ml}$ acid denaturing solution (10\% trichloroacetic acid (TCA) in acetone pre-chilled at $-20^{\circ} \mathrm{C}$, $0.05 \%$ dithiothreitol (DTT) added just before use). Proteins were then precipitated by incubation overnight at $-20^{\circ} \mathrm{C}$. The precipitate was centrifuged at $4^{\circ} \mathrm{C}$ for $45 \mathrm{~min}$ at $21,100 \times \mathrm{g}$, the supernatant discarded and the pellet washed in $200 \mu \mathrm{l}$ rinsing buffer (acetone pre-chilled at $-20^{\circ} \mathrm{C}, 0.05 \%$ DTT added just before use) and incubated at $-20^{\circ} \mathrm{C}$ for $30 \mathrm{~min}$. The mixture was centrifuged at $4^{\circ} \mathrm{C}$ for $20 \mathrm{~min}$ at $21,100 \mathrm{x} \mathrm{g}$, the supernatant discarded and the pellet washed two more times in rinsing buffer before drying on ice and storage at $-80^{\circ} \mathrm{C}$ for later use. Total protein concentrations were determined with the BioRad Bradford protein assay kit (Bio-Rad, Mississauga, ON, Canada) according to the manufacturer's instructions.

\subsection{4 iTRAQ Analysis}

iTRAQ analysis was performed on proteins extracted from triticale stigmas at the BCP (early) and TCP (late) stages (Figure S1). Proteins $(200 \mu \mathrm{g})$ from each stage within each of three biological replicates (A, B and C) were aliquoted, split into two equal technical replicates of $100 \mu \mathrm{g}$, air-dried using the GeneVac EZ-2 solvent evaporator (SP Scientific, Warminster, PA, USA) and sent to the Plate-forme protéomique, Centre Génomique de Québec (http://proteomique.crchul.ulaval.ca/en/) for protein digestion, iTRAQ labeling, and high performance liquid chromatography (HPLC)-tandem mass spectrometry (MS)/MS as follows. 
Protein pellets were resuspended in a solution of $4.5 \mathrm{M}$ urea and $1 \%$ sodium deoxycholate. Equal amounts of protein, $20 \mu \mathrm{g}$ for each replicate, were used for iTRAQ labeling. Proteins were first reduced with $7.5 \mathrm{mM}$ DTT for $30 \mathrm{~min}$ at $37^{\circ} \mathrm{C}$ and then alkylated with $14.3 \mathrm{mM}$ iodoacetamide (IAA) for $20 \mathrm{~min}$ at room temperature (RT). The urea concentration was diluted to $2 \mathrm{M}$ with water and samples were digested with trypsin (Sequence Grade Modified, Promega Corporation, Madison, WI, USA) using a 1:30 ratio overnight at $37^{\circ} \mathrm{C}$. After digestion, peptides were acidified to precipitate deoxycholate and then purified with a C18 Empore filter (Sigma-Aldrich Canada Co., Oakville, ON, CA) and lyophilized. Dried peptides were dissolved in $30 \mu 10.5 \mathrm{M}$ triethylammonium bicarbonate (TEAB) and labeled with iTRAQ label reagent (Applied Biosystems, Foster City, CA, USA). Four-plex labeling (Figure S2) was performed for $2 \mathrm{~h}$ at RT in the dark. Labeled peptides were combined in one tube and cleaned using HLB cartridges (Waters Corporation, Milford, MA, USA) and dried with a SpeedVac (Thermo Fisher Scientific, San Jose, CA, USA).

iTRAQ samples were fractionated (24 fractions) using high $\mathrm{pH}(\mathrm{pH} 10)$ reversedphase (RP) HPLC using the Agilent Technologies Inc. 1200 HPLC system (Agilent Technologies Inc., Santa Clara, CA, USA). The samples were loaded in $10 \mathrm{mM}$ ammonium bicarbonate buffer ( $\mathrm{pH} 10)$ at $25 \mu \mathrm{l} / \mathrm{min}$ on a Zorbax Extend $\mathrm{C} 18$ microbore RR column (Agilent Technologies Inc.) and eluted with a gradient of 5-35\% of B (A: Water, $10 \mathrm{mM}$ ammonium bicarbonate, B: 90\% Acetonitrile, $10 \mathrm{mM}$ ammonium bicarbonate) for $60 \mathrm{~min}$, and then $35-70 \%$ of $\mathrm{B}$ in $24 \mathrm{~min}$. The fractions were pooled as described in Yang et. al. (2012). Fractions were then dried with the SpeedVac and resuspended in $0.1 \%$ formic acid for analysis by MS. For each experiment, $750 \mathrm{ng}$ of 
peptide samples were injected and separated by online RP nanoscale capillary LC (nanoLC) and analyzed by electrospray ionization (ESI) MS/MS. The experiments were performed with a Dionex UltiMate 3000 nanoRSLC chromatography system (Thermo Fisher Scientific/Dionex Softron GmbH, Germering, Germany) connected to an Orbitrap Fusion $^{\mathrm{TM}}$ Tribrid $^{\mathrm{TM}}$ mass spectrometer (Thermo Fisher Scientific) equipped with a nanoelectrospray ion source. Peptides were trapped at $20 \mu 1 / \mathrm{min}$ in loading solvent $(2 \%$ acetonitrile, $0.05 \%$ trifluoroacetic acid (TFA)) on a $5 \mathrm{~mm}$ x $300 \mu \mathrm{m} \mathrm{C} 18$ pepmap cartridge pre-column (Thermo Fisher Scientific/Dionex Softron $\mathrm{GmbH}$ ) for $5 \mathrm{~min}$. The pre-column was then switched online with a self-made $50 \mathrm{~cm}$ x $75 \mu \mathrm{m}$ internal diameter separation column packed with ReproSil-Pur C18-AQ 3- $\mu \mathrm{m}$ resin (Dr. Maisch, HPLC $\mathrm{GmbH}$, Ammerbuch-Entringen, Germany) and the peptides were eluted with a linear gradient from 5-40\% solvent B (A: $0.1 \%$ formic acid, B: $80 \%$ acetonitrile, $0.1 \%$ formic acid) for $90 \mathrm{~min}$ at $300 \mathrm{~nL} / \mathrm{min}$. Mass spectra were acquired using the data-dependent acquisition mode using Thermo XCalibur software version 3.0.63. Full scan mass spectra (350 to $1800 \mathrm{~m} / \mathrm{z}$ ) were acquired in the Orbitrap using an automatic gain control (AGC) target of $4 \times 10^{5}$, a maximum injection time of $50 \mathrm{~ms}$ and a resolution of 120000 . Internal calibration using lock mass on the $\mathrm{m} / \mathrm{z} 445.12003$ siloxane ion was used. Each MS scan was followed by acquisition of fragmentation MS/MS spectra of the most intense ions for a total cycle time of $3 \mathrm{~s}$ (top speed mode). The selected ions were isolated using the quadrupole analyzer in a window of $1.6 \mathrm{~m} / \mathrm{z}$ and fragmented by Higher energy Collision-induced Dissociation (HCD) with 45\% of collision energy. The resulting fragments were detected by the Orbitrap at resolution 60000 with an AGC target of $1 \times 10^{5}$ and a maximum injection time of $120 \mathrm{~ms}$. Dynamic exclusion of previously 
fragmented peptides was set for a period of $20 \mathrm{~s}$ and a tolerance of $10 \mathrm{ppm}$. MS/MS peak lists (MGF files) were generated using Proteome Discoverer version 1.4.0.288 (Thermo Fisher Scientific). MGF samples were analyzed using Mascot version 2.5.2 (Matrix Science, London, UK) to search the TAX_Triticeae_147389 database (https://www.uniprot.org/uniprot/?query=triticeae\&sort=score) assuming trypsin digestion with two missed cleavages being allowed, a fragment ion mass tolerance of 25 PPM, and a parent ion tolerance of 10.0 PPM. Carbamidomethylation of cysteine was specified as a fixed modification. Deamidation of asparagine and glutamine, oxidation of methionine and iTRAQ 4-plex of lysine, tyrosine and the N-terminus were specified as variable modifications.

Scaffold version 4.7.5 (Proteome Software Inc., Portland, OR, USA) was used to validate MS/MS based peptide and protein identifications. Peptide identifications were accepted if they could be established at greater than $95.0 \%$ probability by the Scaffold Local FDR algorithm. Protein identifications were accepted if they could be established at greater than $95.0 \%$ probability and contained at least 2 exclusive peptides. Protein probabilities were assigned by the protein prophet algorithm (Nesvizhskii et. al., 2003). Proteins that contained similar peptides and could not be differentiated based on MS/MS analysis alone were grouped to satisfy the principles of parsimony. Proteins sharing significant peptide evidence were grouped into clusters.

Scaffold Q+ version 4.7.5 (Proteome Software Inc.) was used to quantitate iTRAQ protein identifications. Only proteins identified (at the thresholds described above) across all replicates were used for quantification. Normalization was performed iteratively (across samples and spectra) on intensities, as described in Oberg et. al., 2008. 
Medians were used for averaging. Spectra data were log-transformed, pruned of those matched to multiple proteins and those missing a reference value, and weighted by an adaptive intensity weighting algorithm. Statistically significant differentially abundant proteins were determined by applying the Mann-Whitney test with unadjusted significance level $\mathrm{p} \leq 0.05$ corrected by Benjamini-Hochberg to $\mathrm{p} \leq 0.02988$. Proteins showing $\mathrm{a} \geq 1.5$ fold difference in abundance were considered to be differentially abundant.

\subsubsection{Global Mass Spectrometry Analysis}

Global analyses were performed on proteins extracted from mature triticale stigmas and pollen grain (Figure S3). Approximately $1 \mathrm{mg}$ of triticale mature stigma and pollen proteins were each resuspended in $6 \mathrm{M}$ urea, $50 \mathrm{mM}$ Tris $(\mathrm{pH}$ 8) to a final concentration of $6 \mu \mathrm{g} \mathrm{ml}^{-1}$. The samples were then agitated at RT at $650 \mathrm{RPM}$ for $1 \mathrm{~h}$ and then placed on a rotor for $5 \mathrm{~h}$ for further agitation. The proteins were then reduced by the addition of DTT to a final concentration of $10 \mathrm{mM}$ and then agitated at $56{ }^{\circ} \mathrm{C}$ at 650 RPM for 45 min. Alkylation of the proteins was performed by the addition of $0.4 \mathrm{M}$ IAA solution in $50 \mathrm{mM}$ ammonium bicarbonate to a final concentration of $14 \mathrm{mM}$. The samples were then agitated on a rotator for $30 \mathrm{~min}$ at RT in the dark. Trypsin was added to the samples and the proteins digested according to manufacturer's instructions (Promega Corporation) with agitation at $750 \mathrm{RPM}$ at $37^{\circ} \mathrm{C}$ overnight. The tryptic digests were adjusted to $\mathrm{pH} 10$ with ammonium formate and $1 \mathrm{ml}$ for each sample was individually injected and separated offline into 60 fractions via high $\mathrm{pH}$ (pH 10) RPHPLC using a C18 Hypersil gold column (Thermo Fisher Scientific). The experiments 
were performed with the ÄKTA purifier (Amersham Biosciences, Little Chalfont, UK) and the peptides were eluted with a linear gradient from $4-40 \%$ solvent B (A: water, 20 $\mathrm{mM}$ ammonium formate $\mathrm{pH}$ 10; $\mathrm{B}$ : acetonitrile (ACN), $20 \mathrm{mM}$ ammonium formate $\mathrm{pH}$ 10), at a flow rate of $1 \mathrm{ml} / \mathrm{min}$. The fractions were then concatenated into 20 fractions by combining fractions $14,34,54,74 ; 15,35,55 \ldots$ and the pooled samples were then airdried in a GeneVac EZ-2 solvent evaporator (SP Scientific) and sent to the Plate-forme protéomique, Centre Génomique de Québec (http://proteomique.crchul.ulaval.ca/en/) for inline low pH RP-HPLC MS/MS. Peptide samples $(1 \mu \mathrm{g})$ were injected and separated by online RP nanoLC and analyzed by ESI MS/MS as described above with the exception that the peptides were eluted in $35 \mathrm{~min}$ and the linear ion trap was used to detect the peptide fragments after HCD. The fragments were detected in a rapid scan rate with an AGC target of $1 \times 10^{4}$ and a maximum injection time of $50 \mathrm{~ms}$.

A second global MS analysis was performed on mature stigma proteins. Aliquots $(10 \mu \mathrm{g})$ from each of the 6 mature stigma protein samples not used in the iTRAQ analysis, that had already been sent to the Plate-forme protéomique, Centre Génomique de Québec (http://proteomique.crchul.ulaval.ca/en/), were washed 3 times with $50 \mathrm{mM}$ ammonium bicarbonate buffer using an Amicon $3 \mathrm{kDa}$ column. After elution, the samples were vacuum-dried and kept at $-20^{\circ} \mathrm{C}$ until trypsin digestion. Proteins were solubilized in $25 \mu 150 \mathrm{mM}$ ammonium bicarbonate, $1 \%$ sodium deoxycholate and heated at $95^{\circ} \mathrm{C}$ for $5 \mathrm{~min}$. Two micrograms of each sample was reduced with DTT $(0.2 \mathrm{mM})$ at $37^{\circ} \mathrm{C}$ for $30 \mathrm{~min}$ and alkylated with IAA $(0.9 \mathrm{mM})$ at $37^{\circ} \mathrm{C}$ for $20 \mathrm{~min}$. Finally, trypsin $(1 \mu \mathrm{g})$ was added and the samples were incubated overnight at $37^{\circ} \mathrm{C}$. The trypsin reaction was stopped by acidification with a solution of $3 \%$ acetonitrile, $1 \%$ TFA, and $0.5 \%$ acetic 
acid. The peptide samples were combined, purified on a stage tip (C18) and vacuumdried before MS injection. Peptides were resuspended into $0.1 \%$ formic acid for analysis by MS. Peptide samples $(1 \mu \mathrm{g})$ were injected and separated by online RP nanoLC and analyzed by electrospray mass spectrometry (ESI MS/MS) as mentioned above with the exception that the peptides were eluted in $270 \mathrm{~min}$ and the linear ion trap was used to detect the peptide fragments after HCD. The fragments were detected in a rapid scan rate with an AGC target of $1 \times 10^{4}$ and a maximum injection time of $50 \mathrm{~ms}$.

All MS/MS peak lists (MGF files) were generated as above and all global experiments were analyzed using Mascot version 2.5.1 and X! Tandem (The GPM, https://thegpm.org; version CYCLONE (2010.12.01.1)). Mascot and X! Tandem were used to search the TAX_Triticeae_147389 database and the TAX_Viridiplantae_33090 database (https://www.uniprot.org/uniprot/?query=viridiplantae\&sort=score) assuming trypsin digestion with two missed cleavages being allowed. They were searched with a fragment ion mass tolerance of $0.60 \mathrm{Da}$ and a parent ion tolerance of $10.0 \mathrm{ppm}$. Carbamidomethylation of cysteine was specified as a fixed modification. Oxidation of methionine, deamidation of asparagine and glutamine, dehydration of the N-terminusglutamic acid to pyro-glutamate of the N-terminus, and ammonia-loss of the N-terminusglutamine to pyro-glutamate of the $\mathrm{N}$ terminus were specified as variable modifications. Scaffold version 4.7.5 (Proteome Software Inc.) was used to validate MS/MS based peptide and protein identifications as described above. Proteins with a single peptide match were validated as previously described (Nazemof et. al. 2014). 


\subsubsection{General Data Analysis}

Proteins identified from the various searches were combined as follows. The proteins identified from searches using the Triticeae database were combined to nonTriticeae proteins identified exclusively from searches using the Viridiplantae database. For the stigma, proteins identified in at least one sample from the iTRAQ analysis were also combined with proteins identified from the global Triticeae and Viridiplantae searches provided that their matching Brachypodium orthologs were absent from the list of Brachypodium orthologs of proteins from the initial Triticeae and Viridiplantae searches.

Proteins were annotated based on the gene ontology (GO) classification system (www.geneontology.org) using the annotation software Blast2Go version 4.1.9 (www.blast2go.com, BioBam, Valencia, Spain). BlastP was performed against the NCBI non-redundant protein sequence (nr) database using the top 20 hits at an expectation value (E) threshold of $\leq 1 \times 10^{-25}$. The annotation step was configured with an annotation cutoff of 55 and GO weight of 5. Annotations were also retrieved from domain/motif information in a sequence-wise manner using the Blast2Go InterPro scan and merged with existing annotations. Finally, annotations were augmented using the Blast2Go Annex program. The annotations were categorized based on their biological processes. Proteins with no significant matches and proteins matching known proteins or domains but without an associated biological process were grouped together under the category "Unclassified and Unknown". A BLAST was performed with the protein sequences against The Arabidopsis Information Resource (TAIR) protein database using the top hit at an E-threshold of $\leq 1 \times 10^{-5}$ and annotated manually. Fisher's exact term 
enrichment analysis was performed on Arabidopsis orthologs at an E-threshold of $\leq 1 \mathrm{x}$ $10^{-25}$ using the AmiGo2 online tool (http://amigo.geneontology.org/amigo) with the background set as the PANTHER Biological Process GO annotated Arabidopsis thaliana proteome (Mi et. al., 2017). The p-values were adjusted for multiple tests with the Benjamini-Hochberg procedure and only terms with an adjusted $p$-value of $<0.05$ were considered to be statistically enriched or under-represented.

\subsection{Results and Discussion}

Pollen tube growth in the pistil is initially autotrophic and therefore uses stored nutrients in the pollen grain. However, growth of the pollen tube through the pistil subsequently becomes heterotrophic and depends upon the stigma and style providing nutrients, materials, and energy-rich metabolites to the growing pollen tube for sustained growth and guidance through the pistil to the ovary (McInnis et. al., 2006; Yue et. al., 2014). An in depth examination and comparison of the proteomes of the mature stigma and pollen grain will therefore provide insights into both the shared and distinct proteins that are required for their upcoming interaction.

\subsubsection{Functional Classification of Triticale Mature Stigma and Pollen Proteins}

This study identified a total of 11533 and 2977 triticale stigma and pollen proteins, respectively. To our knowledge, this represents the largest Triticeae stigma and pollen proteomic datasets to date. Proteins were annotated using the gene ontology (GO) classification system and placed into 14 broad Biological Processes categories (Figure 3.2). Proteins identified within the mature stigma and pollen displayed a similar 


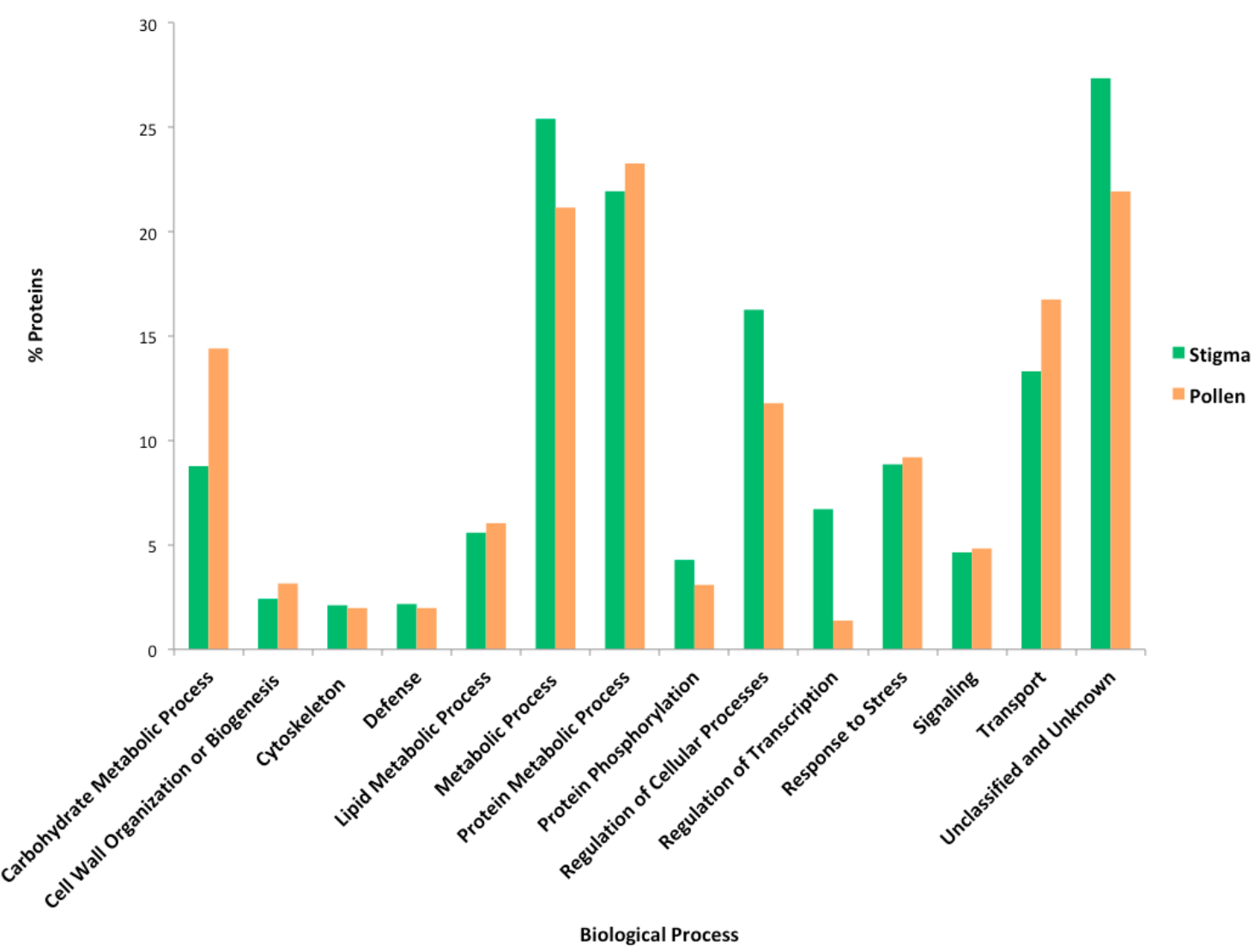

Figure 3.2. Comparison of the functional distribution of the triticale mature stigma and pollen proteomes. 
distribution trend across all 14 categories and most of the proteins fell within 6 categories namely, Carbohydrate Metabolic Process, Metabolic Process, Protein Metabolic Process, Regulation of Cellular Process, Response to Stress, and Transport. This similarity between the pollen and stigma proteomes may reflect a predominance of proteins involved in shared fundamental processes or in some cases in common objectives such as supporting pollen tube growth. There was however a noticeably higher proportion of pollen proteins in the Carbohydrate Metabolic Process and Transport categories, whereas a higher proportion of stigma proteins was found in the categories Metabolic Process, Regulation of Cellular Process, and Regulation of Transcription (Figure 3.2). The global stigma protein distribution pattern was similar to that obtained in a previous proteomic study of the triticale stigma (Nazemof et. al., 2014) even though about 5X as many proteins were identified in this study. However, nearly 20X as many protein kinases (PKs) and transcription factors (TFs) were identified within this investigation (Table S3.1).

The triticale pollen and stigma proteome datasets were then analyzed to uncover the Biological Processes that were enriched or under-represented with respect to the entire plant. The resulting total number of statistically enriched and under-represented sub-categories in pollen and stigma were somewhat similar, with 720 for the stigma and 648 for pollen. There were 25 major Biological Processes that contained enriched subcategories in the stigma proteome and 23 in the pollen proteome, with the most number of enriched sub-categories for the stigma being within Development, Gene Expression, Nucleobase-containing Compound Metabolism, Protein Metabolism, Response to Stimulus, RNA Metabolism, and Transport, whereas the most number of enriched pollen 
sub-categories were in Development, Nucleobase-containing Compound Metabolism, Protein Metabolism, Response to Stimulus, and Transport (Figure 3.3). This data can also be displayed showing the number of unique enriched Biological Processes subcategories highlighting the many differences found in the pollen and stigma proteomes (Figure 3.4). For example, the stigma had noticeably more enriched unique subcategories within Cell Cycle, Chromosome Organization, Defense Response, Development, Gene Expression, Nucleobase-containing Compound Metabolism, Protein Metabolism, Response to Stimulus, and RNA Metabolism, whereas the pollen contained more enriched unique sub-categories within Carbohydrate Metabolism, Cytoskeleton Organization and Transport (Figure 3.4). A description of all the main categories, their enriched sub-categories and constituent proteins is beyond the scope of this chapter; the discussion will therefore focus mostly on the more prevalent categories.

\subsubsection{Cell Cycle}

The Cell Cycle category was enriched in both the stigma and the pollen proteomes, with the former showing more enriched sub-categories and having a significantly higher (6X) proportion of proteins (Figure 3.3, Tables S3.4 and S3.5). One hundred and twenty-five of the stigma Cell Cycle proteins were involved in cell division and included 26 proteins involved in sister chromatid segregation (Table S3.4). Some triticale protein kinases (PKs) in this category have orthologs in Arabidopsis that have been demonstrated to also have a role in cell elongation and patterning such as cyclindependent kinase (CDK) F;1 (Takatsuka et. al., 2009), MAP3Ke1 (Chaiwongsar et. al., 2012), and ERECTA (Cai et. al., 2017). Seventy-eight other proteins of the Cell Cycle 


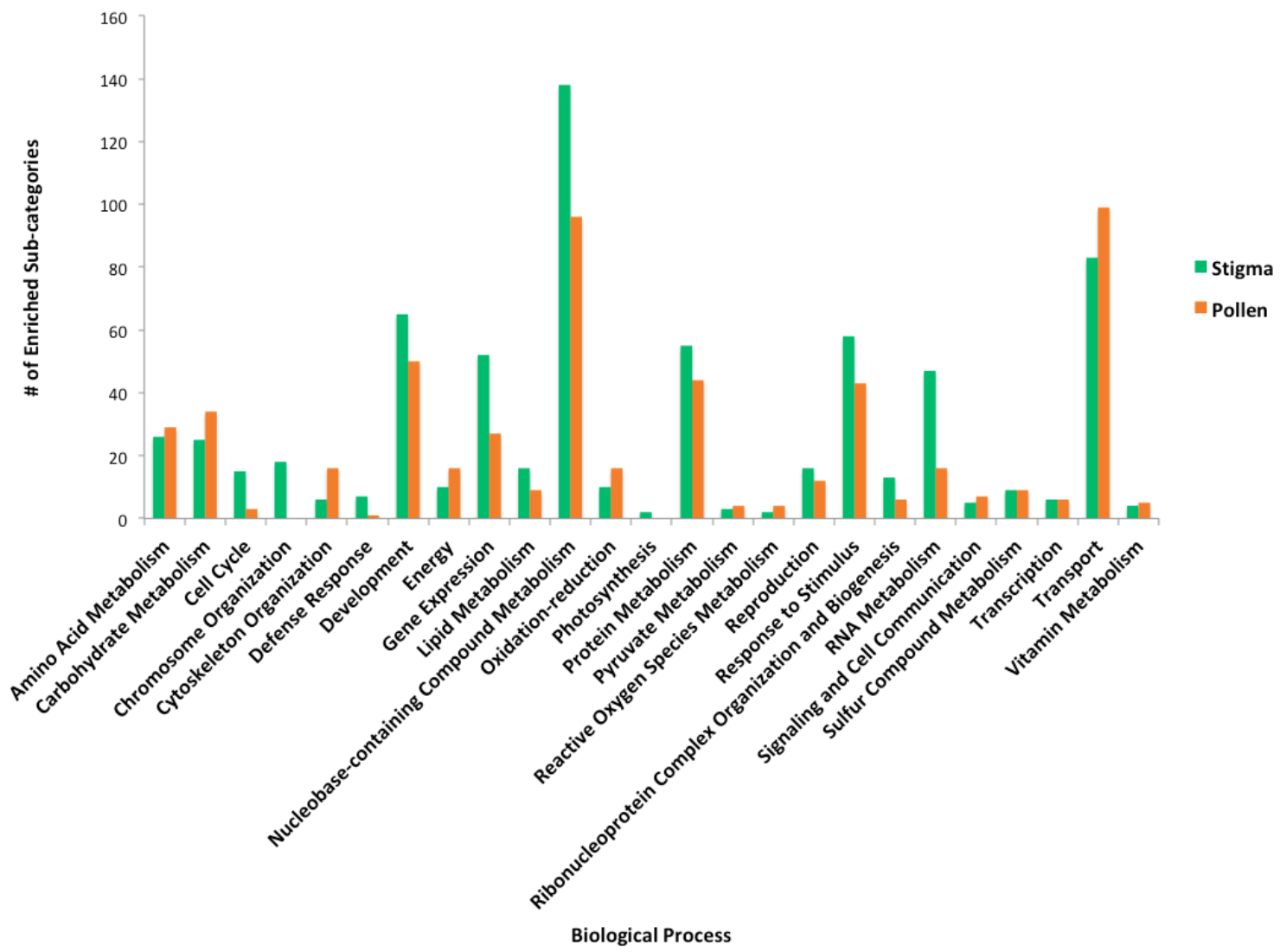

Figure 3.3. Comparison of the number of enriched functional sub-categories of the triticale mature stigma and pollen proteomes. 


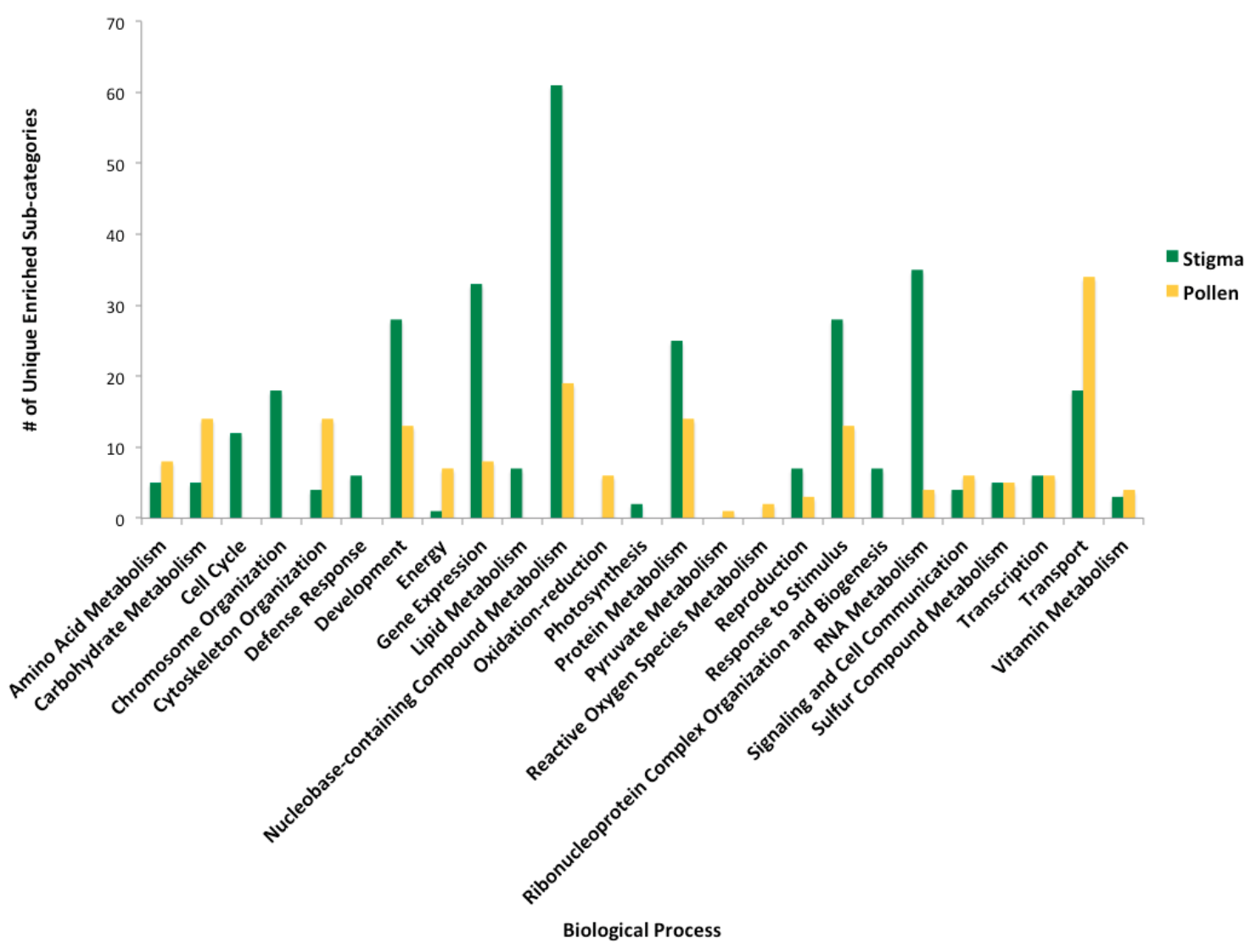

Figure 3.4. Comparison of the number of unique enriched functional sub-categories of the triticale mature stigma and pollen proteomes. 
category also displayed roles in cell elongation with some like dynamin-related protein (DRP) 1A having been shown to be important during stigmatic papillae expansion (Kang et. al., 2003; Konopka and Bednarek, 2008). This enrichment in proteins involved in cell division and elongation may enable the plumose stigma to produce papillate structures right up to maturity (Figure 3.1b).

As mentioned above, the pollen proteome contained fewer proteins involved in the Cell Cycle process. In triticale, the mature pollen grain is tricellular and therefore no further cell division should occur (Grobei et. al., 2009). In addition, pollen tube growth requires polarized tip growth of the vegetative cell that is strictly dependent on polar exocytosis, and does not require cell division for elongation (Qin and Yang, 2011). Accordingly, 9 of the pollen proteins in the Cell Cycle category also have roles in cell elongation such as STOMATAL CYTOKINESIS DEFECTIVE (SCD) 1 required for cell cytokinesis in addition to polarized cell expansion (Falbel et. al., 2003) and whose mutant is infertile (Paez Valencia et. al., 2016), the translationally controlled tumor protein (TCTP) 1 involved in cell expansion during pollen tube and root hair growth (Berkowitz et. al., 2008), and 2-dehydro-3-deoxyphosphooctonate aldolase 1 (KDSA1) that plays a role in pollen tube elongation via its role in the biosynthesis of 2-keto-3-deoxy-D-mannooctulosonic acid Kdo which is a component of the pectic polysaccharide rhamnogalacturonan II (Delmas et. al., 2008) (Table S3.5).

\subsubsection{Chromosome Organization}

Unlike the pollen grain, the stigma is still metabolically active and is presumably undergoing processes involved in the cell cycle and the regulation of transcription 
(described below). These processes require the reorganization of the chromosome (Nagano et. al., 2017; Meyer et. al., 2018). Accordingly, Chromosome Organization and its sub-categories were only enriched in the stigma (Figure 3.4) with 36 proteins involved in chromatin remodeling and 74 proteins involved in histone modification (Table S3.4). Some of these proteins have orthologs in Arabidopsis shown to have an impact on stigma development including chromatin remodeling (CHR) 6/PICKLE (PKL)/GYMNOS (GYM) (Eshed et. al., 1999), chromatin remodeling complex subunit R (CHR) 3/SPLAYED (SYD) (Wagner and Meyerowitz, 2002), the leucine-rich repeat (LRR)containing protein TONSOKU (TSK)/BRUSHY (BRU) 1/MGOUN (MGO) 3 (Guyomarc'h et. al., 2006), and like heterochromatin protein (LHP) 1-interacting factor (LIF) 2 (Latrasse et. al., 2011).

\subsubsection{Development}

The Development category contained many enriched sub-categories in both the stigma and pollen proteomes (Figure 3.3), however, significant differences in the uniquely enriched sub-categories were observed (Figure 3.4) and these reflect their specialization. With respect to the stigma, 21 out of 28 unique sub-categories were related to epidermal cell development, post-embryonic plant organ development and flower development, while for the pollen, 9 out of the 13 unique sub-categories pertained to root hair and pollen tube development (Table S3.4 and S3.5).

The developmental differences between the stigma and pollen are further exemplified by the transcription factors (TFs) and protein kinases (PKs) that were identified in their respective proteomes. TFs can play an important role in the regulation 
of genes that modulate cell differentiation, as well as tissue and organ development (Gonzalez, 2016). Additionally, plant growth involves a plethora of processes and the activity of many of the proteins involved is controlled by phosphorylation performed by PKs (Laurie and Halford, 2001). Therefore, for brevity, the discussion of the pollen and stigma Development category will focus primarily on examples of relevant TFs and PKs, although many other proteins involved in their development can be found in Tables S3.4 and S3.5.

There were 12X more TFs identified within the Development category in the stigma than in the pollen (Table S3.4 and S3.5). The B3 domain TFs made up the largest group of TFs identified in the stigma and included several orthologs to members of the Arabidopsis family of Auxin Response Factors (ARFs) (Table S3.4). Auxin is a plant hormone that plays a critical role in most major growth responses throughout development such as organogenesis, tissue differentiation, root initiation, and tropism, to name a few, and is involved in cellular processes like extension, division and differentiation (Li et. al., 2016). Auxin regulates distinct developmental processes through ARFs which control the expression of target auxin response genes (Li et. al., 2016). Some examples include ARF2 which is important to male and female gametophyte development in Arabidopsis (Liu et. al., 2018). It was also shown to act maternally to control seed growth by restricting cell proliferation (Schruff et. al., 2006) and $\operatorname{arf} 2$ mutants have defects in apical hook formation in etiolated seedlings and have increased seed size (Guilfoyle and Hagen, 2007). Three orthologs to ARF6 were also identified and share redundant functions with ARF2 in cell division within the developing embryo (Rademacher et. al., 2011). ARF6 is also involved in hypocotyl elongation and 
the TF complex containing ARF6 can integrate multiple signaling pathways involving other hormones such as gibberellic acid, brassinosteroids and abiotic response signaling cascades, to regulate hypocotyl elongation developmentally or in response to the environment (Chandler, 2016). Two ARF8 orthologs were also identified, and the Arabidopsis arf6arf8 double mutant was female sterile and its stigmatic papillae did not elongate (Wu et. al., 2006). ARF8 is also involved in hypocotyl growth in response to light and interacts with BIGPETAL (also identified) to synergistically repress cell division and cell expansion in early and late stages, respectively, of petal development (Varaud et. al., 2011). ARF8 restricts premature ovary growth before fertilization (Goetz et. al., 2006) and along with ARF2 it acts as linkers between the ethylene and auxin signaling pathways (Wu et. al., 2006). Lastly, ARF8 also plays a role in pollen tube guidance towards the ovules (Kay et. al., 2013). Other members of the B3 domain TFs identified included orthologs of NGATHA (NGA) 1 and 2 TFs which have redundant roles in style and stigma development (Alvarez et. al., 2009).

Several members of the homeobox TF family were also identified in the stigma proteome (Table S3.4). The homeobox has a characteristic DNA-binding homeodomain (HD) and is involved in processes like cell communication and differentiation (Gonzalez, 2016). In line with the fact that stigma papillae are epidermal cells (Lolle and Pruitt, 1999), several of the homeobox TFs identified have Arabidopsis orthologs shown to have strong expression in the epidermis such as HOMEODOMAIN GLABROUS (HDG) 1 , HDG5 and HDG11 (Nakamura et. al., 2006). Along with the orthologs to PROTODERMAL FACTOR (PDF) 2 that are required for epidermal cell differentiation 
and whose mutant has a wooly trichome phenotype (Abe et. al., 2003; Yang et. al., 2011), these factors have combined roles in flower development (Kamata et. al., 2013).

The next largest group of TFs identified in the stigma (Table S3.4) was the MADS-box gene family known to be involved in floral development (Saedler et. al., 2001). Orthologs to the Arabidopsis homoeotic TFs such as AGAMOUS (AG), APETALA (AP) 1, AP3, SEPALLATA (SEP) 2 SEP3, as well as PISTILLATA (PI) were all identified in the stigma and are thus likely to play a role in its development. For example, SEP2 and SEP3 are required to determine the identity of petals, stamens and carpels, and have been implicated in Arabidopsis stigma development (Pelaz et. al., 2000; Favaro et. al., 2003). Many other types of TFs identified in the stigma can be found in Table S3.4 including some shown to play a role in stigma development such as LEUNIG (LUG) (Chen et. al., 2000), CRABS CLAW (CRC) (Alvarez et. al., 1999) and SEUSS (SEU) (Franks et. al., 2002). It is important to note that many of these proteins mentioned above (as well as many other developmental regulators identified) have never been shown to be present in the Triticeae stigma previously.

Only 3 TFs were identified in the Development category in the pollen (Table S3.5); the Arabidopsis ortholog to the TOPLESS-RELATED (TPR) 1/MODIFIER OF SNC1 (MOS) 10 that is involved in embryo patterning and auxin signaling (Zhu et. al., 2010), as well as the GATA TFs GATA24/zinc-finger protein expressed in inflorescence meristem (ZIM)-like (ZML) 1 involved in photoprotection (Shaikhali et. al., 2012) and GATA3 of unknown function.

Unlike the TFs, the proportion of PKs within the Development category was similar between the stigma and pollen (Table S3.4 and S3.5). Consistent with the 
enrichment of the Cell Cycle category described above for the stigma, several PKs that control the successive phases of the cell cycle were identified in the stigma. These kinases typically consist of a catalytic cyclin-dependent kinase subunit and an activating cyclin subunit, and are called cyclin-dependent kinases (CDKs) (Mironov, 1999) (Table S3.4). Examples of Arabidopsis orthologs identified in the stigma proteome include CDKA;1 which is linked to proliferation competence and functions in controlling Sphase entry and mitosis (Nowack et. al., 2012; Hemerly et. al., 1993). It is also important for phosphatidylcholine biosynthesis and couples the rate of phospholipid biosynthesis and endomembrane biogenesis to cell cycle progression (Craddock et. al., 2017). Importantly, dominant-negative CDKA;1 expression results in defects in epidermis continuity i.e. a loss of individual cells and occurrence of gaps between anticlinal walls of neighboring pavement cells (Borowska-Wykręt et. al., 2013). Orthologs to CDKC;1 involved in leaf growth and trichome development (Cui et. al., 2007) and CDKD1;1 important to female gametogenesis (Takatsuka et. al., 2015) were also identified.

Several MAP kinases were also part of the stigma proteome (Table S3.4) and included orthologs of Arabidopsis MPK6 important to pollen tube guidance (Guan et. al., 2014) and ovule development (Wang et. al., 2008), MKK4 and MKK5 involved in stomatal development (Wengier et. al., 2018), as well as RAF-like (RAF) 28 involved in the relay of positional information for cellular patterning in the root epidermis (Liu et. al., 2013). Other members of these PK families as well as other PK families involved in development can be found in Table S3.4. However, it is also worth mentioning the presence of orthologs of the Arabidopsis SHAGGY-related protein kinases implicated in brassinosteroid signaling such as ASK7/BRASSINOSTEROID-INSENSITIVE (BIN) 
2/ULTRACURVATA (UCU) 1 involved in cell expansion along the proximodistal axis (Perez-Perez et. al., 2002) and ASK1/AtSK11 important to style and stigma epidermal cell fate (Dornelas et. al., 2000).

With respect to the pollen, several members of the Catharanthus roseus RLK1like kinase (CrRLK1L) subfamily were identified within the Development category (Table S3.5). CrRLK1Ls play a central role in regulating fertilization as well as cell expansion mechanisms such as cell elongation and tip growth (Galindo-Trigo et. al., 2016). An example would be ANXUR (ANX) 1 that plays a role in controlling cell wall integrity during pollen tube growth (Boisson-Dernier et. al., 2011). Orthologs were also found to other PKs involved in pollen tube growth including the calcium-dependent PKs CPK17 and CPK34 that transduce $\mathrm{Ca}^{2+}$ signals to increase the rate of pollen tube tip growth, in addition to facilitating the response to tropism cues (Myers et. al., 2009) (Table S3.5). Root hair growth is similar to that of the pollen tube as it occurs via the polar elongation of a single cell at the tip (Schoenaers et. al., 2017). Accordingly, examples of PKs playing a role in root hair and pollen tube growth included the orthologs to the Arabidopsis receptor-like PKs MARIS (MRI) (Liao et. al., 2016) and MALE DISCOVERER (MDIS) 2 that interacts with voltage-gated potassium channel AKT2 affecting pollen tube guidance and root hair development (Sklodowski et. al., 2017; Wang et. al., 2016), among others (Table S3.5). 


\subsubsection{Nucleobase-containing Compound Metabolism, Gene Expression, RNA}

\section{Metabolism, and Transcription}

There were many processes involved in Nucleobase-containing Compound Metabolism that were enriched within both the stigma and the pollen proteomes, although the stigma had more uniquely enriched sub-categories (Figure 3.3 and 3.4). Nucleobasecontaining compounds are involved in a large number of biological processes required for reproduction including the production of pyrimidine nucleotides which are involved in the synthesis and degradation of sucrose (for energy) as well as UDP-glucose which is the precursor for many cell wall polysaccharides needed for cell wall biosynthesis and modification during pollen tube invasion of the pistil (Zrenner et. al., 2006), $\mathrm{Ca}^{2+}$ and $\mathrm{H}^{+}$ fluxes during pollen tube growth through the use of cyclic nucleotide-gated ion channels (Song et. al., 2009), ATP synthesis for the energy required for pollen tube growth, as well as NADPH synthesis as a co-factor for many biological processes, and GDP/GTPcontaining molecules for signaling and cytoskeleton dynamics required for vesicular trafficking during pollen tube growth (Yue et. al., 2014; Cheung and Wu, 2008). Nucleobase-containing Compound Metabolism also involves RNA Metabolism and Transcription, and many proteins within the Nucleobase-containing Compound Metabolism category also overlap with processes like gene silencing (Table S3.4 and S3.5). It is therefore unsurprising that Nucleobase-containing Compound Metabolism was the category containing the most enriched sub-categories for the stigma and among the largest categories for pollen (Figure 3.3). The stigma contained more unique subcategories involved in pyrimidine-containing compound metabolism and DNA metabolism, while the pollen contained more unique sub-categories involved electron 
transport and in purine-containing compound metabolism like ATP and GMP synthesis. It is expected that the pollen grain would not contain many proteins involved in DNA metabolism as it is metabolically quiescent at this stage (Grobei, et. al., 2009). The stigma, however, is still metabolically active and 207 proteins involved in DNA metabolism were identified (Table S3.4). Given that the stigma contained more subcategories involved in the metabolism of pyrimidine-containing compounds, it's possible that it prioritizes the synthesis of these compounds and maybe the synthesis of materials used for cell wall polysaccharide production. On the other hand, given that pollen tube growth is a highly energetic process (Selinski and Scheibe, 2014), the pollen may prioritize ATP synthesis for energy.

Gene Expression and RNA Metabolism contained enriched sub-categories in the pollen, however a greater number of enriched sub-categories were present in the stigma, and both categories overlapped considerably (Figure 3.3, Tables S3.4 and S3.5). The majority of these processes pertained to post-transcriptional processes like mRNA processing and protein synthesis. For the pollen, this is consistent with the fact that rapid translation of stored transcripts is a prioritized event during germination and the initial stages of pollen tube formation (Grobei et. al., 2009).

The stigma contained a few enriched processes involved in transcription initiation however most of the enriched Transcription sub-categories were regulatory. In fact most of the stigma proteins identified within the Transcription category had roles in negative regulation of transcription possibly suggesting that general transcription is slowing in the maturing stigma (Table S3.4). In both the pollen and stigma, Transcription did not contain many enriched sub-categories and displayed more under-represented sub- 
categories (Table S3.4 and S3.5). The under-represented sub-categories for both the pollen and stigma contained proteins that mostly had roles in RNA biosynthesis. Surprisingly, processes like Positive Regulation of Transcription Initiation from RNA Polymerase II was enriched in the pollen (Table S3.5) and since the mature pollen grain already contains pre-synthesized mRNAs required for the initial stages of reproduction (Obermeyer et. al., 2013), it could be expected that these processes would be underrepresented. Upon closer inspection of the corresponding pollen proteins (Table S3.5), all of them were $26 \mathrm{~S}$ protease regulatory subunits that have been shown to have a dual role in protein catabolism and transcription activation in yeast and humans (Ferdous et. al., 2002). It is possible that these $26 \mathrm{~S}$ subunits are involved in pollen protein metabolism and not directly in transcription. Generally, many of the proteins identified in this study can have multiple roles in various biological processes and as a result are often assigned to multiple gene ontology categories.

\subsubsection{Protein Metabolism}

Protein Metabolism and many of its sub-categories were enriched in both the stigma and pollen proteomes in comparison to the rest of the plant (Figure 3.3). Thirty sub-categories involved in protein synthesis were shared among both the pollen and stigma. The stigma however contained more uniquely enriched sub-categories involved in protein modification (Table S3.4), including proteins involved in Chromosome Organization discussed above. Examples of stigma proteins involved in protein modification included orthologs to the Arabidopsis hydroxyproline Ogalactosyltransferase (GALT) 5 important for pollen tube growth because it glycosylates 
the cell wall structural arabinogalactan proteins that are candidate proteins for pollenpistil adhesion and known to act as a chemo-attractant during pollen tube growth (Rejón et. al., 2016; Dresselhaus and Franklin-Tong, 2013; Showalter and Basu, 2016), prolyl 4hydroxylase (P4H) 4 and $\mathrm{P} 4 \mathrm{H} 1$ important to root hair tip growth as they hydroxylate prolines in the cell wall hydroxyproline-rich glycoproteins (HRGPs) required for cell wall strengthening (Velasquez et. al., 2011, 2015; Deepak et. al., 2010) and Ofucosyltransferase (OFT) 1 involved in pollen tube penetration at the stigma-style interface (Smith et. al., 2018). Many more such proteins can be found in Table S3.4.

The pollen had more sub-categories involved in protein catabolism (Table S3.5). This is consistent with the fact that protein degradation is required for the turnover of short-lived regulatory proteins essential for cytoskeleton organization and of enzymes associated with cell wall organization during pollen tube growth (Sheng et. al., 2006). Furthermore, the ER secretory pathway that is essential for pollen tube growth possesses a quality control mechanism called ERAD (ER-associated degradation) that ensures that only correctly folded, processed and completely assembled proteins can exit and be transported through to the trans Golgi network. Disruption of this degradative mechanism has been shown to decrease pollen tube growth and morphology (Sheng et. al., 2006).

\subsubsection{Response to Stimulus and Defense Response}

Response to Stimulus and many of its sub-categories were enriched in both the stigma and pollen proteomes (Figure 3.3). In fact, approximately $30 \%$ of their proteins were allocated to this category (Table S3.4 and S3.5) and therefore a complete discussion 
of the constituent proteins becomes beyond the scope of this chapter. Nonetheless, the stigma contained more uniquely enriched sub-categories than the pollen (Figure 3.4), with 25 involved in a plethora of responses to different stimuli and stresses which is in line with the fact that as the receptive part of the pistil it needs to be responsive to external stimuli, and is also likely less hermetic than the mature pollen grain. More than 170 TFs were identified in the stigma Response to Stimulus category (Table 3.4). For example, the stigma contained 20 orthologs to TFs from the FAR-RED IMPAIRED RESPONSE (FAR) 1 family (Table S3.4). FAR1 and FAR1-related sequence (FRS) proteins participate in light regulation of development and could also be involved in signal transduction processes of other biotic or abiotic stresses (Lin and Wang, 2004). In Arabidopsis, FAR1 acts downstream of phytochrome A (phyA) to regulate far-red light photomorphogenic development and coordinates the activation of circadian clock evening components. It also has a role in regulating plant immunity by modulating chlorophyll biosynthesis (Wang et. al., 2016). FRS6, for example, acts as a positive regulator in the phyB red and white light signaling pathway controlling flowering time (Lin and Wang, 2004). In fact, there were several TFs involved in light signaling, photomorphogenesis, and circadian rhythm expressed in the stigma including cryptochrome (CRY) 2-interacting BHLH (CIB) 2 (Liu et. al., 2013), LONG HYPOCOTYL (HY) 5 (Nawkar et. al., 2017), B-box domain protein (BBX) 22 (Gangappa and Botto 2014), homeobox-leucine zipper protein (HB) 16 (Wang et. al., 2003), and TEOSINTE BRANCHED 1, CYCLOIDEA and PROLIFERATING CELL FACTOR (TCP) 2 (He et. al., 2016), to name but a few. It will be interesting to further investigate the importance of light to the development of the fully enclosed triticale 
plumose stigma. The pollen exhibited few TFs in the Response to Stimulus category. However, the two largest groups of proteins (after the PKs) were GTPases and proteases (Table S3.5). Both the pollen and the stigma contained many proteins involved in stress responses (Table S3.4 and S3.5).

As in pollen, PKs made up the largest group of proteins within the stigma Response to Stimulus category with 230 proteins (Table S3.4). More than one third of the PKs were receptor-like protein kinases and although some have no known function, several were orthologs to Arabidopsis proteins involved in pathogen response, while others were involved in various signaling processes such as the response of the cysteinerich receptor-like kinase (CRK) 6 to ROS (Idanheimo et. al., 2014), in pollen tube guidance like AT1G06840 (Chen et. al., 2014) and in pollen tube reception such as FERONIA (FER) (Chapman and Goring, 2010). A number of the stigma receptor-like PKs have also been shown to function in hormonal signaling pathways such as the transmembrane kinase (TMK) 1 which is part of an auxin perception complex (Chen and Yang 2014) and is important to pavement cell development (Xu et. al., 2014), although most were involved in brassinosteroid signaling such as HERCULES (HERK) 1 involved in cell expansion (Nissen et. al., 2016) or BRASSINOSTEROID INSENSITIVE (BIN) 1/BRI1 involved in pollen tube growth (Ye et. al., 2010). In Arabidopsis, brassinosteroids promote pollen germination and tube growth (Vogler et. al., 2014) and this may also account for the presence of multiple orthologs to brassinosteroid-signaling kinase (BSK) 1, BSK2, BSK3 in the stigma (Table S3.4). The next two largest classes of PKs within the stigma Response to Stimulus category were MAP PKs discussed above and calcium-dependent PKs (Table S3.4). Calcium-dependent PKs perceive intracellular 
changes in $\mathrm{Ca}^{2+}$ concentration and translate them into specific phosphorylation events to initiate further downstream signaling processes like environmental biotic and abiotic stress, and immune response signaling (Schulz et. al., 2013). Examples include orthologs to the Arabidopsis CDPK6 that is responsive to abiotic and biotic stress, and is involved in stomatal closing (Qin et. al., 2016), CDPK7 that was shown to negatively control the cellular abundance of aquaporin PIP1 and other membrane transporters (Li et. al., 2015) and is involved in stomatal movement (Hubbard et. al., 2015), as well as CDPK2 which mediates the $\mathrm{Ca}^{2+}$-dependent inhibition of $\mathrm{K}^{+}$channels and participates in the regulation of pollen tube growth (Zhao et. al., 2013). CDPK2 can also promote ethylene biosynthesis (Luo et. al., 2014), regulate abscisic acid signaling (Zhu et. al., 2007), and positively regulate the PAMP (pathogen-associated molecular pattern)-induced ROS burst (Boudsocq et. al., 2010).

With respect to the pollen, most of the uniquely enriched Response to Stimulus sub-categories pertained to responses to sugar/carbohydrate and to ROS (Table S3.5). Given the high energetic requirements for pollen tube growth, it is expected that there would be response proteins involved in sugar metabolism and transport such as orthologs to the Arabidopsis hexokinase (HXK) 1 and sugar transport protein (STP) 1 , as well as malectin domain-containing receptor-like kinases like ANXUR (Boisson-Dernier et. al., 2013) and RLK5 (Ruprecht et. al., 2016). The fact that the pollen Response to Stimulus category contains more uniquely enriched sub-categories involved in responses to ROS is in keeping with the fact that ROS play important roles in signaling (Steinhorst and Kudla, 2013), as well as assisting in pollen tube breach during germination by guiding apertural tube outgrowth (Potocký et. al., 2012). Examples of PKs involved in ROS signaling 
include orthologs to the MAP kinase (MPK) 1 that is activated by ROS like $\mathrm{H}_{2} \mathrm{O}_{2}$ (Colcombet et. al., 2016) and MPK8 which helps to maintain ROS homeostasis (Takahashi et. al., 2011) (Table S3.5). Other examples of PKs important to pollen tube growth include AGC kinase (AGC) 1.5 and 1.7 (Zhang et. al., 2009), and CBLinteracting protein kinase (CIPK) 12 (Steinhorst et. al., 2015) (Table S3.5).

The stigma contained a higher proportion of defense response proteins than the pollen (Table S3.4 and S3.5) and one of the uniquely enriched sub-categories was Defense Response to Virus. Since the pollen grain exine wall consists of the highly resistant biopolymer sporopollenin, which can withstand degradation by microbes and insects (Edlund, et. al., 2017), this may explain why proteins involved in the defense response are not as prevalent within the pollen grain as in the stigma. Nonetheless, more than 259 proteins involved in defense including PKs and TFs were identified in both the stigma and pollen proteomes (Table S3.4 and S3.5).

\subsubsection{Transport}

Transport was enriched in both the stigma and the pollen, and included numerous enriched sub-categories involved in nuclear transport, mitochondrial transport, vesicular transport, water transport (for the stigma), protein transport, and hydrogen transport (Table S3.4 and S3.5). The pollen had a higher number of transport sub-categories than the stigma (Figure 3.3). With respect to the stigma, transport plays a facilitating role in pollen hydration, germination and pollen tube growth by delivering water, nutrients, enzymes, cell wall polysaccharide constituents and other materials to the pollen contact site. However, Vesicular transport categories like Golgi Vesicle Transport, Endocytosis 
and Exocytosis are required by the pollen to drive rapid pollen tube tip growth (Goring et. al., 2017; Cheung and $\mathrm{Wu}, 2008)$. An $\mathrm{H}^{+}$influx at the tip and efflux along the subapical region maintains pollen tube apex acidity (Boavida et. al., 2005). Accordingly, the pollen showed $12 \mathrm{X}$ more enriched sub-categories related to ion and proton transport, and approximately $3 \mathrm{X}$ more channels involved in the transmembrane movement of protons such as autoinhibited $\mathrm{H}(+)$ ATPases and vacuolar H(+) ATPases (Table S3.5).

\subsubsection{Differential Protein Abundance during Triticale Stigma Maturation}

An iTRAQ analysis was performed on triticale stigmas in order to ascertain which proteins had changes in abundance as it matured and prepared to receive the pollen. Out of 3278 proteins identified, 647 were differentially abundant proteins (DAPs), with 305 proteins increasing in abundance (Table S3.6), while 342 proteins decreased in abundance (Table S3.7). The proteins were categorized into the 14 broad Biological Processes categories described above, with the processes that had significantly more higher abundance proteins than lower abundance proteins being Carbohydrate Metabolic Process, Cell Wall Organization or Biogenesis, Defense, Response to Stress, Signaling, and Transport. The categories that contained significantly more lower than higher abundance proteins were Cytoskeleton, Metabolic Process, Protein Metabolic Process, Protein Phosphorylation, and Regulation of Transcription (Figure 3.5). Although many of the proteins that differ in abundance as the stigma matures have roles consistent with stigma function and development, there are numerous examples with no known roles in the stigma (or elsewhere in the plant) whose differential abundance in the stigma may warrant further investigation. 


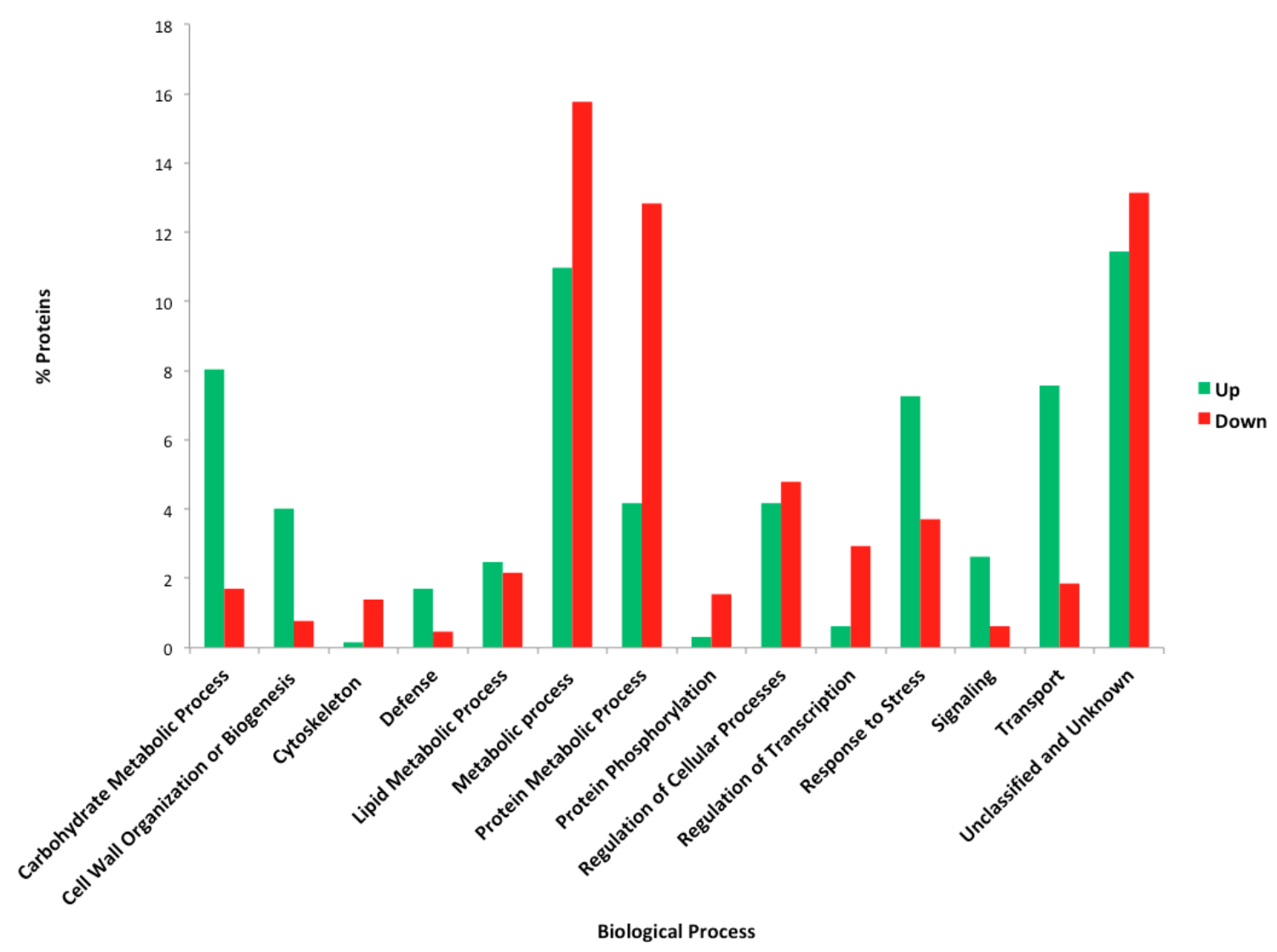

Figure 3.5. Percentage of proteins that increased and decreased in abundance during triticale stigma maturation. 


\subsubsection{Carbohydrate Metabolic Process}

Carbohydrates are the main source of energy during plant reproduction, serve as signaling molecules in pollen germination and tube guidance, and are integral components of plant cell walls (Hirsche et. al., 2017; Mollet et. al., 2013). Twenty-seven percent of the proteins that increased in abundance within Carbohydrate Metabolism overlapped with proteins belonging to Cell Wall organization or Biogenesis, while the rest were primarily involved in defense, and glycolysis (Table S3.6). Energy is required for pollen tube growth (Samuel et. al., 2011) and this likely explains why enzymes involved in glycolysis increased in abundance as the stigma matured (Table S3.6).

\subsubsection{Cell Wall Organization or Biogenesis}

After successful hydration and germination, the pollen tube penetrates the stigmatic cuticle, invades the intercellular space, and grows within the stigma (Dresselhaus et. al., 2011). These are steps that require enzymatic modification of these stigmatic layers. Almost half of the proteins within the Cell Wall Organization or Biogenesis category also fell under the Carbohydrate Metabolism category (Table S3.6). For example, some of the shared enzymes have orthologs in Arabidopsis demonstrated to play a role in cell wall modification such as cellulase (CEL) 3 (Da Costa et. al., 2013), CEL1 (Shani et. al., 2006), UDP-glycosyltransferase (UGT) 72B1 (Lin et. al., 2016), as well as xylanase (XYN) 1 shown to facilitate pollen germination (Suen and Huang 2007). The remaining proteins consisted mostly of peroxidases and some expansins (Table S3.6). 


\subsubsection{Response to Stress}

Forty-seven stress response-related proteins increased in abundance as the stigma matured and 21 were involved in response to oxidative stress (Table S3.6). The stigma, as the receptive part of the pistil needs to be responsive to external stimuli. However, the stigmatic surface of the pistil is only receptive to the pollen for a limited period of time and high levels of peroxidase activity mark the stigma's readiness for pollination (McInnis et. al., 2006). Furthermore, the stigmas of Senecio squalidus were shown to generate significant amounts of ROS and hydrogen peroxide $\left(\mathrm{H}_{2} \mathrm{O}_{2}\right)$ within their papillae (McInnis et. al., 2006). ROS are postulated to be involved in loosening the stigma cell wall to allow penetration and growth of pollen tubes. They could possibly be also involved in defense against pathogen attack (McInnis et. al., 2006). In addition, small increases in temperature were observed when stigmas from Arabidopsis were dusted with pollen, which is consistent with catalase's exothermic decomposition of $\mathrm{H}_{2} \mathrm{O}_{2}$ (Edlund et. al., 2017). Accordingly, 9 peroxidases, 3 thioredoxins, 2 catalases, and a superoxide dismutase had elevated protein levels in the mature stigma (Table S3.6).

\subsubsection{Defense}

Invasion of the stigma by the pollen tube creates a wound and likely renders the stigma more susceptible to pathogens. Some defense-related proteins increased in abundance in the mature stigma including orthologs to the Arabidopsis hevein-like preproprotein (HEL), non-specific lipid-transfer protein (LTP) 3, cystatin (CYS) 5, and a glucan endo-1,3-beta-glucosidase (Table S3.6). 


\subsubsection{Signaling}

Nearly half of the proteins that had elevated protein levels within the Signaling category were orthologs of Arabidopsis Rab GTPases and ADP-ribosylation factors with some known to be involved in vesicular trafficking such as RABD2A (Heard et. al., 2015) or seed storage protein accumulation such as RABG3A and RABG3D (Ebine et. al., 2014), but with no demonstrated role in the stigma (Table S3.6). Interestingly, the TF auxin response factor (ARF) 6 involved in stigmatic papillae elongation (Wu et. al.,2006) and the protein kinase somatic embryogenesis receptor kinase (SERK) 1 involved in multiple processes including epidermal cell patterning (Meng et. al., 2015) both had decreased abundance in the mature stigma (Table 3.7).

\subsubsection{Transport}

In the Transport category, four times as many proteins were increased than decreased in abundance (Figure 3.5) and the largest group of proteins consisted of lipid transfer proteins with no known role in the stigma, although they may possibly be involved in cuticle formation. Some of the proteins that had elevated levels were involved in vesicular transport such as the Arabidopsis orthologs to ECHIDNA (ECH) and alpha-soluble NSF attachment protein (ASNAP), known to be important to pollen germination and tube growth (Fan et. al., 2014; Xu et. al., 2013). As mentioned previously, transport within the pistil fulfills the requirements associated with pollen hydration, germination and sustained tube growth and guidance (McInnis et. al., 2006). For example, the protein level of four aquaporins, likely required for water transport during pollen hydration, increased as the stigma matured (Table S3.6). Additionally, 
numerous proteins involved in calcium $\left(\mathrm{Ca}^{2+}\right)$ ion and proton transport increased in abundance. Following pollination, three cytosolic $\mathrm{Ca}^{2+}$ level increases (following pollen hydration, prior to pollen germination and during pollen tube penetration) in the apical region of Arabidopsis papillae have been observed (Chapman and Goring, 2010). The

$\mathrm{Ca}^{2+}$ transporters that had elevated protein levels in the triticale stigma could perhaps facilitate this process. Most of the proteins involved in proton transport that increased in abundance, were coupled to ATP synthesis (Table S3.6) which is needed to provide energy rich metabolites to drive pollen tube growth (Yue et. al., 2014). Orthologs to two V-type proton ATPase subunits involved in the maintenance of cytosolic $\mathrm{pH}$ homeostasis also increased in abundance. Cytosolic pH can regulate actin cytoskeletal dynamics, which is important for vesicular transport (Cheung and $\mathrm{Wu}, 2008$ ).

\subsubsection{Cytoskeleton}

There were more stigma proteins involved in cytoskeletal dynamics that decreased than increased in abundance (Figure 3.5). Some of these proteins were involved in microtubule-based processes and included orthologs to microtubuleassociated protein (MAP) 65-1 and MAP 70-2 proteins (Table S3.7). This is consistent with previous observations indicating that microtubule depolymerization in the stigmatic papilla is necessary for compatible pollen acceptance (Samuel et. al., 2011).

\subsubsection{Metabolic Process}

There were more proteins that decreased than increased in abundance within the Metabolic Process category (Figure 3.5). Approximately 50\% of these proteins were 
involved in nucleic acid metabolism such as DNA metabolic processes like DNA synthesis, replication and DNA repair (Table S3.7). Proteins involved in posttranscriptional processes like RNA processing and gene silencing also had lower levels of abundance in the mature stigma (Table S3.7). Given that these processes were part of enriched categories in the stigma (see above), this likely indicates that selected proteins within these processes decrease in abundance as the stigma matures.

\subsubsection{Protein Metabolic Process and Protein Phosphorylation}

More proteins involved in Protein Metabolic Processes were lower in abundance than higher, in the mature stigma (Figure 3.5) and the largest group of proteins that decreased in abundance consisted of ribosomal proteins. This may signify a reduction of translation as the stigma matures although very few other proteins involved in translation had decreased levels. Some of the other proteins had roles in development such as three orthologs to the Arabidopsis ubiquitin-like-specific protease (ULP) 2B that plays a role in megagametogenesis (Liu et. al., 2017), and ARGONAUTE (AGO) 1 which is involved in carpel development (Reyes-Olalde et. al., 2013) (Table S3.7).

There were more proteins involved in Protein Phosphorylation that decreased than increased in abundance as the stigma matured (Figure 3.5). The majority of the proteins were PKs and included SERK1 mentioned above, as well as inflorescence meristem receptor-like kinase (IMK) 2 also involved in epidermal cell patterning (Barre, 2016) (Table S3.7). The roles of the other PKs in the stigma or the reasons they had decreased abundance remain to be investigated. 


\subsubsection{Regulation of Transcription}

Several TFs decreased in protein levels including the orthologs to the Arabidopsis NGA2 and SEP3 important to stigma development, and HDG11 involved in epidermal cell and pollen development (Khosla et. al., 2014), discussed above, as well as corepressors TOPLESS (TPL) and TPL-related (TPR) 2 which have multiple roles in development (Espinosa-Ruiz et. al., 2017) (Table S3.7). Other TFs such as WHIRLY (WHY) 2, alfin-like (AL) 6, bZIP18, bZIP39 and transcription repressor La protein (LA) 1, all of which have no known function in the stigma, decreased in protein abundance (Table S3.7). Conversely, KNOTTED-like from Arabidopsis thaliana (KNAT) 3 increased in abundance as the stigma matured (Table S3.6). The knat3knat4knat5 triple mutants are female sterile (Furumizu et. al., 2015).

\subsubsection{Brassica vs. Triticale Comparison}

The Brassiceae and Triticeae stigmas and pollen grains display very evident morphological differences (Figure 2.1 and 3.1) and it may be anticipated that distinctions would occur in the molecular processes underlying their development. Nevertheless, in both cases dry stigmas covered in unicellular papillae are called upon to support pollination by tricellular pollen grains and thus might also be expected to share many functional requirements in their interactions. In order to gain some insight in both these shared and distinct processes, the $B$. carinata and triticale stigma and pollen proteomes were compared. 


\subsubsection{The Global Proteome}

As mentioned previously, 7703 and 5608 B. carinata stigma and pollen proteins (Chapter 2), as well as 11533 and 2977 triticale stigma and pollen proteins were identified, respectively. The proportions of proteins identified that fell within the 14 broad functional categories were similar between the $B$. carinata and triticale stigma proteomes (Figure 3.6). However, there was a noticeably higher proportion of $B$. carinata stigma proteins belonging to the Defense, Regulation of Cellular Process, Response to Stress, and Signaling categories than in triticale. The reasons for some of these differences are unknown. However, given that the triticale flower is enclosed within the glumes and its stigma is further protected by the palea and lemma, which constitute physical barriers to biotic and abiotic elements, the stigma may not need as many proteins having roles in Defense and Response to Stress as the stigma of $B$. carinata which is exposed once the flower opens.

Like the stigma, the proportions of proteins identified within the 14 broad functional categories were also similar between the $B$. carinata and triticale pollen proteomes (Figure 3.7). However, the $B$. carinata pollen had a noticeably higher proportion of proteins within the categories Regulation of Cellular Process and Response to Stress, while the triticale pollen proteome had a noticeably higher proportion of proteins within the Carbohydrate Metabolic Process and Protein Metabolic Process categories. The reasons for these differences are unknown. 


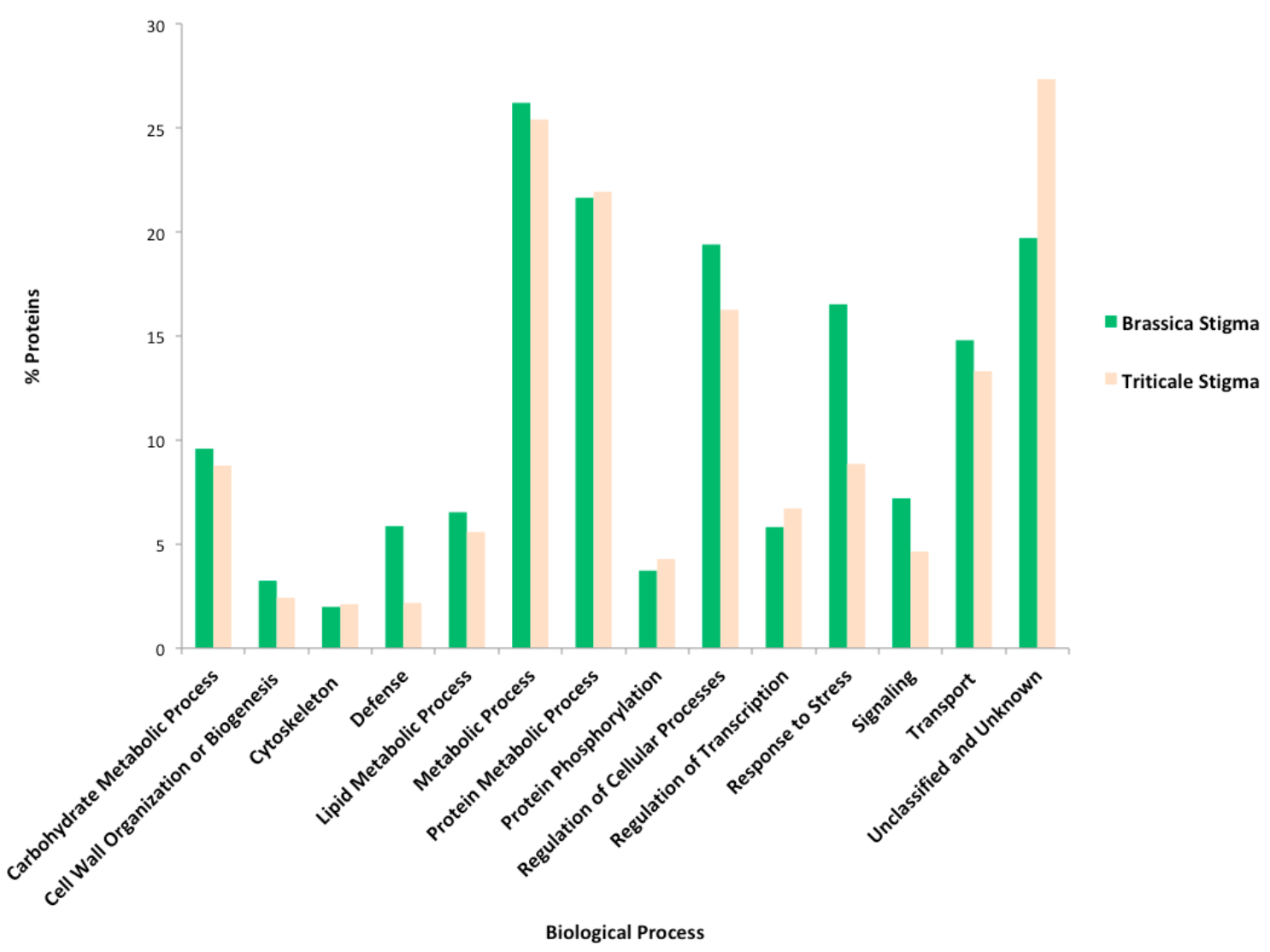

Figure 3.6. Comparison of the functional distribution of $B$. carinata and triticale mature stigma proteomes. 


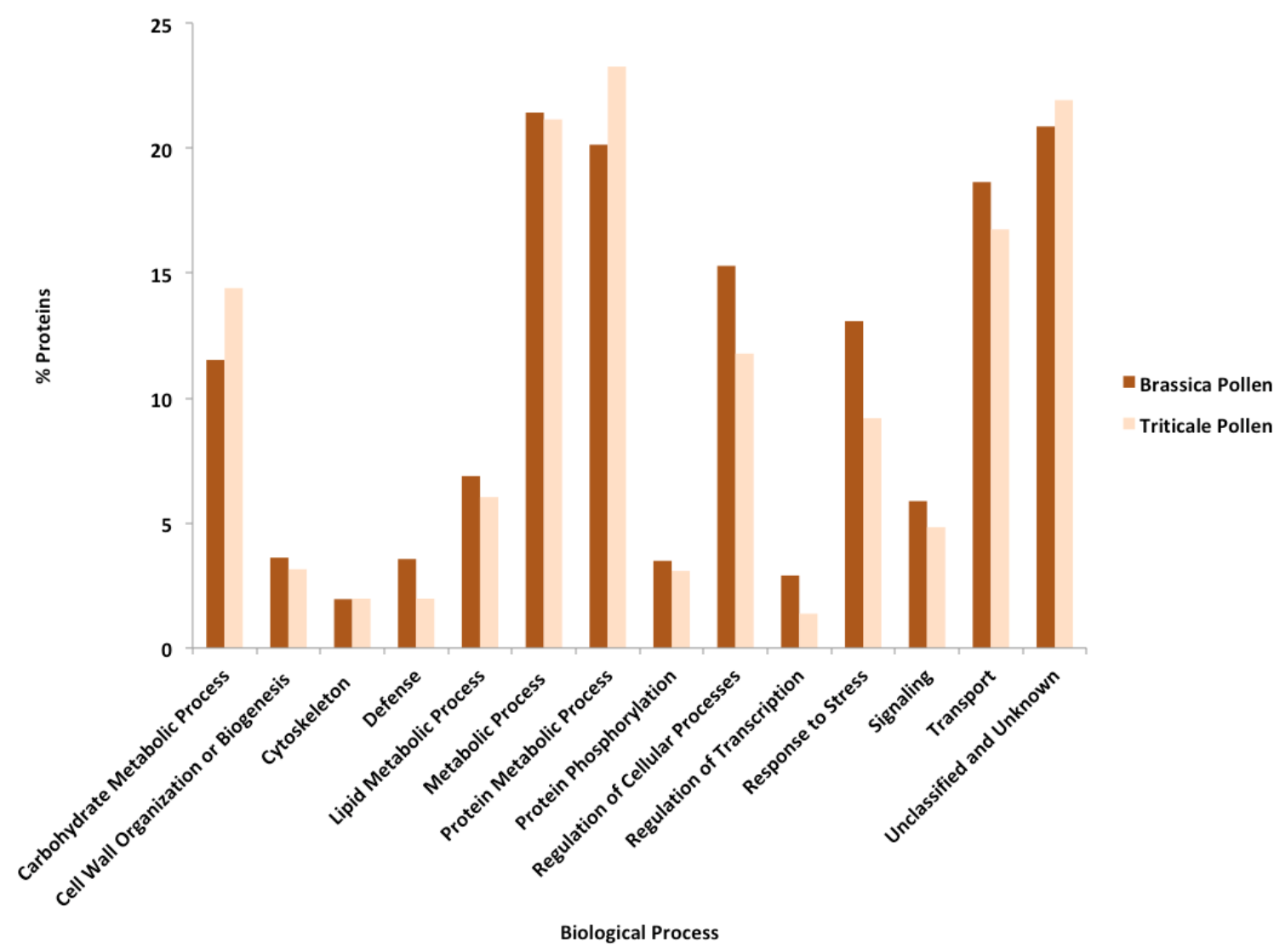

Figure 3.7. Comparison of the functional distribution of $B$. carinata and triticale mature pollen proteomes. 


\subsubsection{Functional Enrichment}

The proportion of proteins belonging to enriched and under-represented Biological Processes from the triticale stigma and pollen were compared to that of $B$. carinata (Figure 3.8 and 3.9). There was very little variation in the proportion of proteins observed in the different categories between the species and it is likely that this overall similarity in protein enrichment is due to a convergence in basic as well as specialized functions in the pollen and stigma. However, upon closer examination of the proteins identified within each category, some differences in the types of proteins carrying out these similar functions could be observed and a few examples will be provided to illustrate this point. For example, the triticale stigma contained 6 orthologs to TCP TFs (Table S3.1) while no TCPs were identified in the B. carinata stigma (Table S2.1). As mentioned above, TCPs regulate a wide variety of plant developmental processes and defense responses via the stimulation of the biosynthetic pathways of hormones such as brassinosteroid and jasmonic acid, and can integrate internal developmental cues with external environmental stimuli for the orchestration of plant development (Li, 2015). For instance, TCP2 regulates the expression of NGA mentioned above (Ballester et. al., 2015). Similarly, several orthologs to FAR1-related TFs were identified in the triticale stigma (Table S3.1), whereas no FAR1 or FRS orthologs were detected in the B. carinata stigma (Table S2.1). As mentioned above, these TFs are postulated to have roles in regulating flowering time and the circadian rhythm in response to light stimuli (Lin and Wang, 2004; Siddiqui et. al., 2016). On the other hand, several orthologs to Arabidopsis LTPs were identified in both the triticale and B. carinata stigma. However, while the 


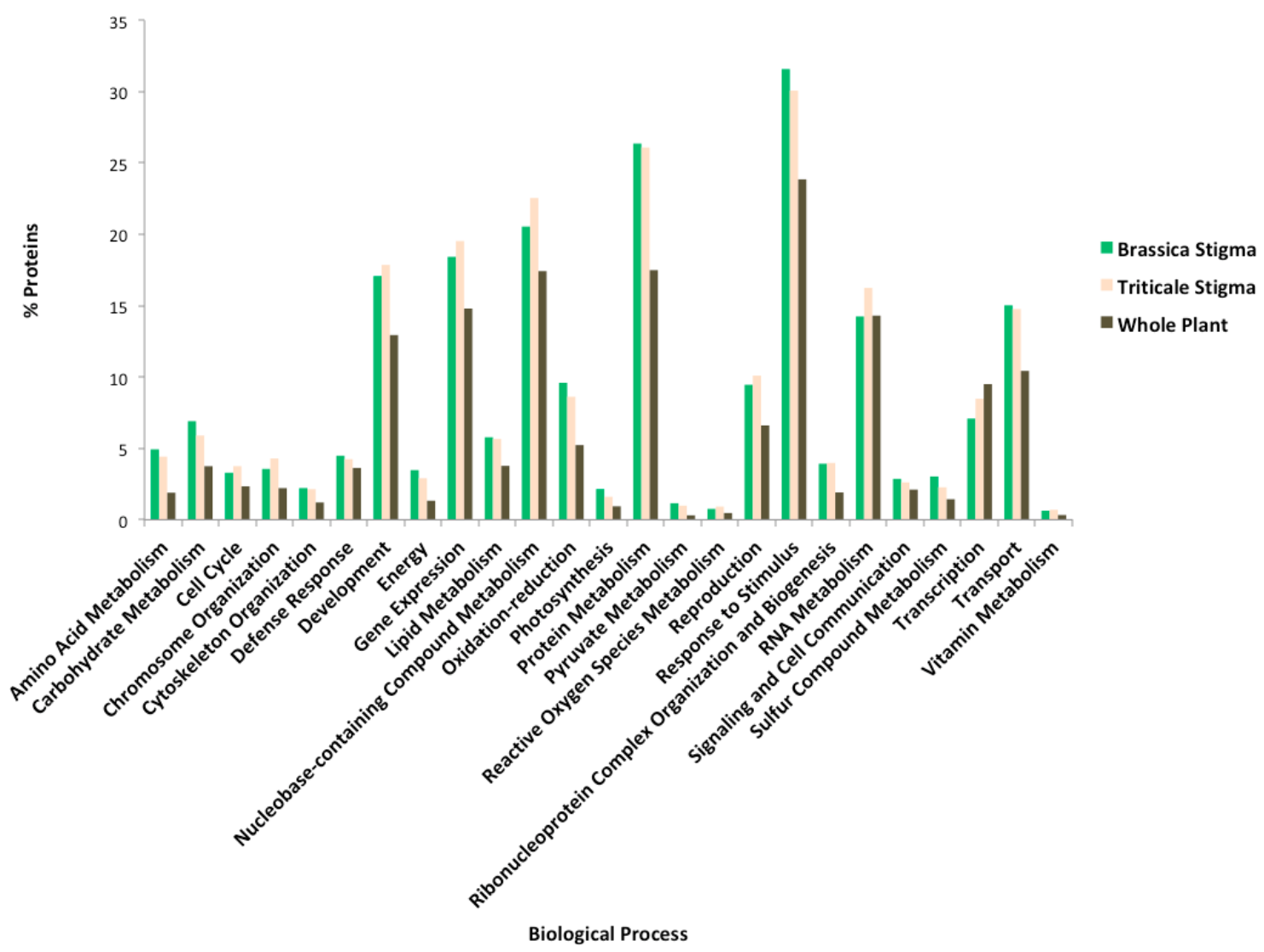

Figure 3.8. Comparison of the enriched and under-represented functional categories of $B$. carinata and triticale mature stigma proteomes. 


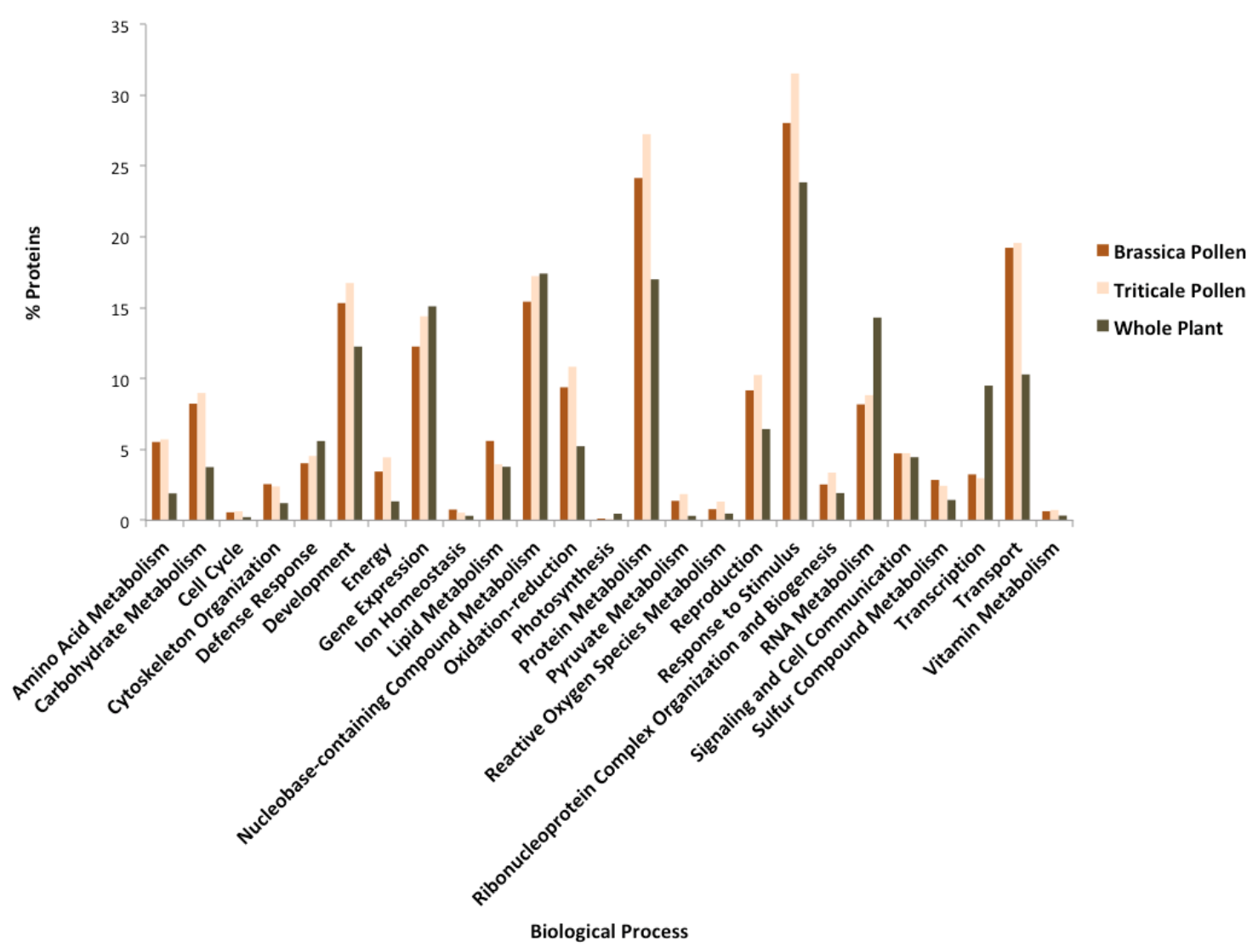

Figure 3.9. Comparison of the enriched and under-represented functional categories of $B$. carinata and triticale mature pollen proteomes. 
majority of the B. carinata LTP orthologs had roles in wax or cuticle development, most of the triticale LTP orthologs had no known function (Table S2.1 and S3.1).

Some other differences within the proteomes of the stigmas of B. carinata and triticale were observed. For example, similar to a previous study done on B. napus and triticale (Nazemof et. al., 2016) B. carinata was found to contain a higher proportion of proteins involved in glucosinolate metabolism and photosynthesis than triticale (Table S2.1 and S3.1). This is expected since glucosinolates are metabolites characteristic of the Brassicaceae (Alexander et. al., 2008) and the B. carinata stigma is green (Figure 2.1b) unlike the colorless triticale stigma (Figure 3.1b).

For the pollen, most of the differences between the species represented proteins found mostly within B. carinata and although some of these differences may be due to about twice as many Brassica proteins being identified, the examples provided below all exceed this ratio. For example, several orthologs of DEAH-box and DExH-box RNA helicases involved in RNA metabolism were identified within B. carinata, while none were identified in triticale (Table S2.1 and S3.1). Similarly, 15 orthologs of the Arabidopsis galacturonosyltransferase (GAUT) which has a role in pectin biosynthesis (Atmodjo et. al., 2011) and can influence pollen tube growth (Wang et. al., 2013) were identified in B. carinata, whereas only one was identified in triticale (Table S2.1 and S3.1). Lastly, several tapetal oleosins involved in pollen hydration (Rejón et. al., 2016) were observed in Brassica but absent from triticale (Table S2.1 and S3.1), which is expected as tapetal oleosins are limited to the Brassicaceae family (Rejón et. al., 2016). 


\subsubsection{Differential Protein Abundance in the Maturing Stigma}

The DAPs identified during triticale stigma maturation in this study were compared to those obtained for B. carinata (Chapter 2) and since these results were presented previously, this discussion will focus mainly on the differences between the two species. The DAPs were placed within the 14 broad functional categories mentioned above. In general, $B$. carinata had a higher proportion of proteins that increased in abundance in most categories, with the exception of the Transport category (Figure 3.10). A noticeable difference in the proportion of proteins attributed to the categories was observed between the species in Defense, Lipid Metabolic Process, Metabolic Process, Protein Metabolic Process, Protein Phosphorylation, Regulation of Cellular Process, and Response to Stress. Across all the aforementioned categories, there seemed to be a bias towards proteins involved in response to stimuli as might be expected of mature stigma cells standing in readiness (Table S2.6 and S3.6).

With respect to proteins that decreased in abundance, $B$. carinata had a higher proportion of these proteins in most categories with the exception of Metabolic Process, Protein Metabolic Process, Protein Phosphorylation, and Regulation of Transcription (Figure 3.11). There also seemed to be a difference in protein proportion between the species within Defense, Lipid Metabolic Process, Protein Metabolic Process, and Response to Stress. Unlike the stigma proteins discussed above that had elevated levels, many of the proteins having a lower abundance in these categories showed functional differences between the two species (Table S2.7 and Table S3.7). Metabolic Process, which contained proteins primarily involved in the metabolism of nucleic acids, and 


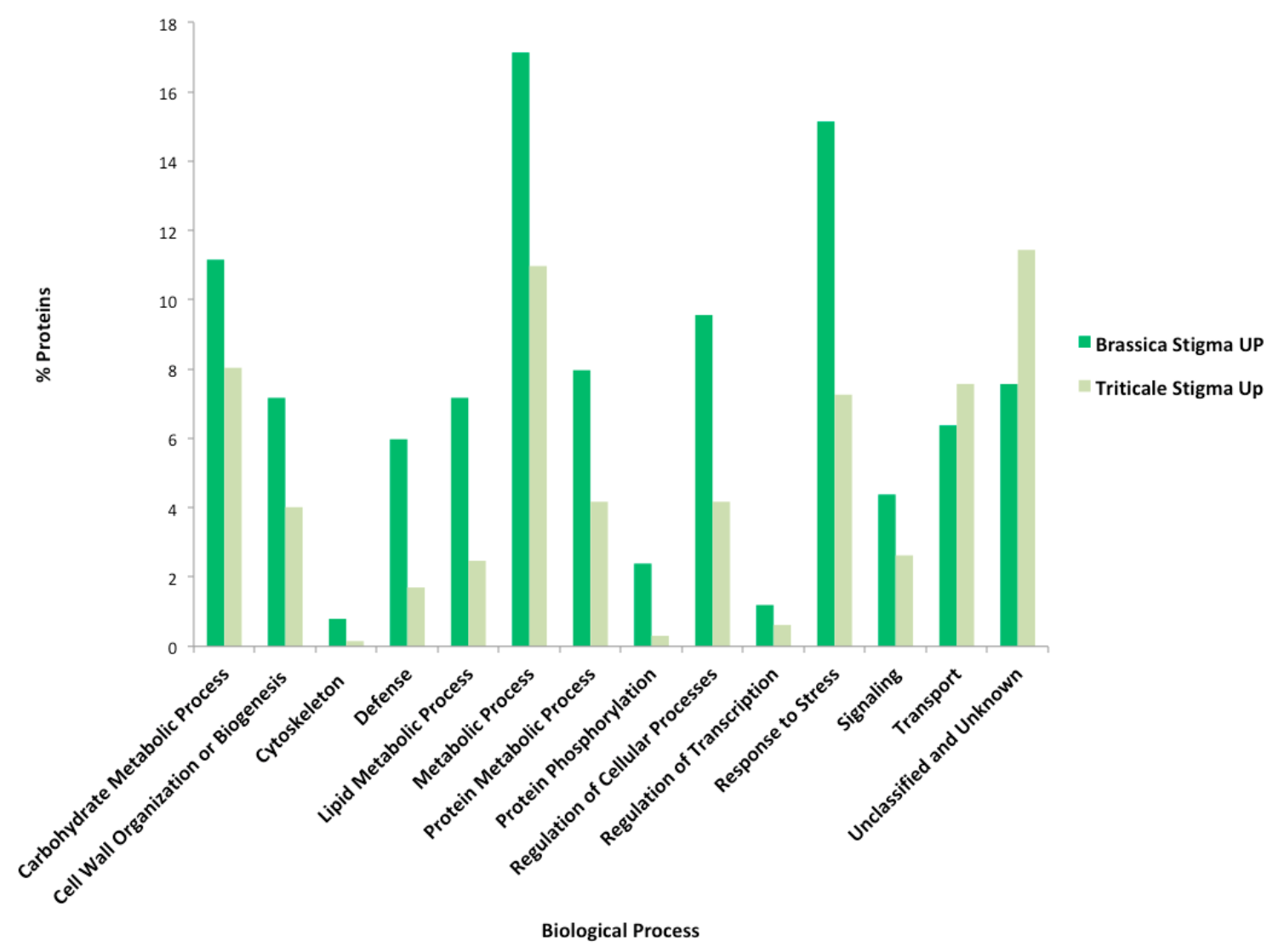

Figure 3.10. Comparison of the number of proteins that increased in abundance during stigma maturation for B. carinata and triticale. 


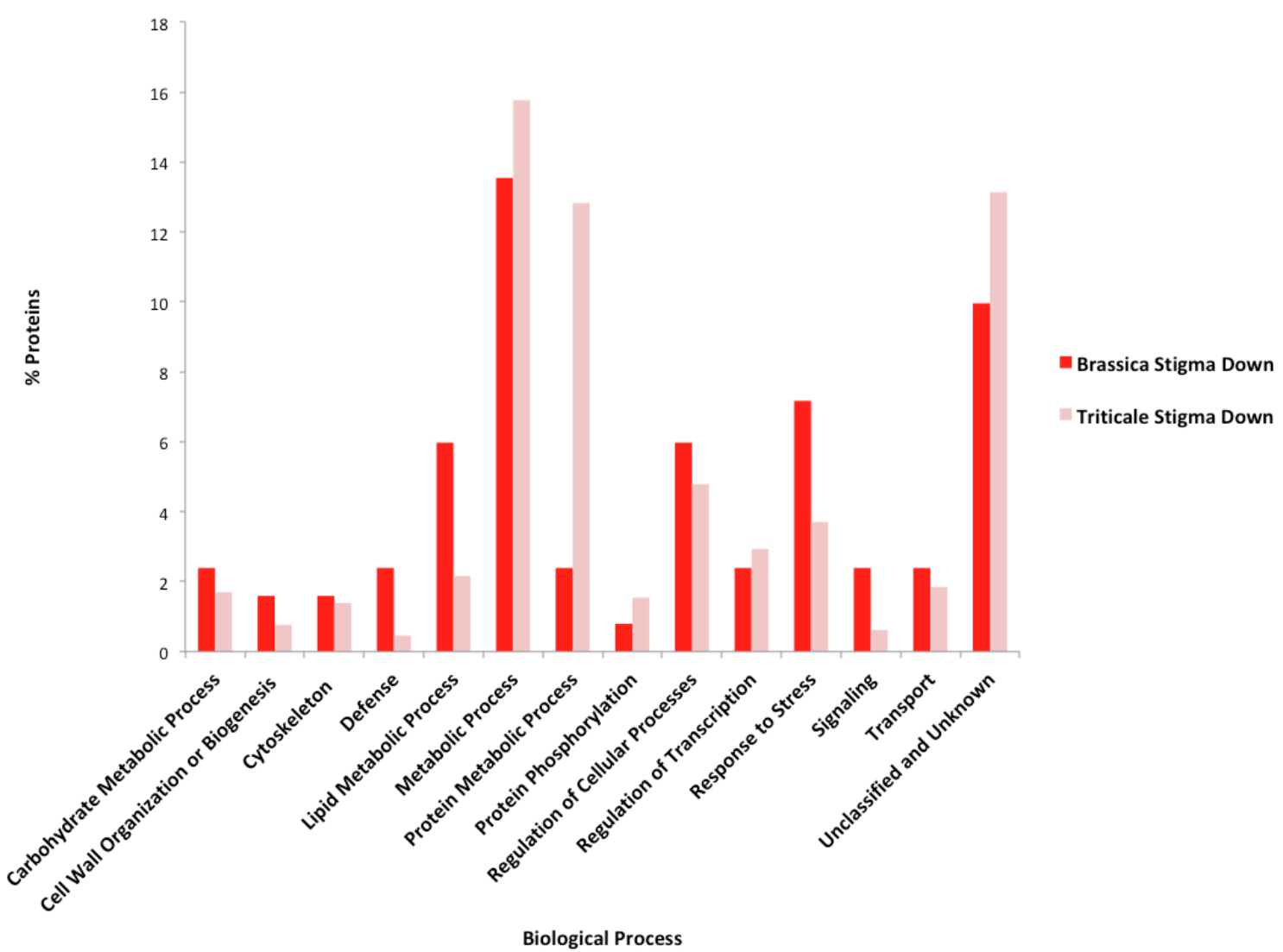

Figure 3.11. Comparison of the number of proteins that decreased in abundance during stigma maturation for B. carinata and triticale. 
Lipid Metabolic Process where mostly proteins involved in wax biosynthesis and cuticle development decreased in abundance, were the exception.

Within the Response to Stress category, approximately $50 \%$ of the proteins that decreased in abundance in B. carinata had responsive roles to abiotic stimuli, while in triticale there was a 50\% skew towards proteins involved in DNA repair (Table S2.7 and Table S3.7). Within Regulation of Transcription, the triticale proteins that had lower protein levels had a stronger functional bias towards developmental proteins (Table S2.7 and Table S3.7). The most significant difference was observed in Protein Metabolic Process where triticale had a 5-fold higher proportion of proteins with a decrease in abundance than B. carinata (Figure 3.11). Upon further examination, almost half of these triticale proteins were involved in gene expression and a large proportion were ribosomal proteins as mentioned above. Very few proteins with roles in protein metabolism decreased in abundance in B. carinata and the ones that had lower protein amounts were involved in transcription, hormone signaling and the proteolysis of propeptides from storage proteins (Table S2.7 and Table S3.7). Although the reasons behind some of these differences are not evident, these disparities between the two species may point to interesting avenues of future research.

\subsection{Conclusion}

Within this study, an extensive proteomics reference data set identifying 11533 and 2977 mature triticale stigma and pollen proteins respectively, was reported, and included novel proteins with no known roles in the stigma, pollen, or elsewhere in the entire plant. Additionally, 647 proteins were found to change in abundance as the stigma 
reached maturation. Detailed analysis of the enriched and under-represented biological processes revealed significant differences between the pollen and the stigma that reflect their specialization. Conversely, a proteomic comparison of the triticale and B. carinata stigmas or pollen showed little functional variation despite their morphological differences, suggesting a conservation of biological processes across taxa, as well as the possibility that the morphological differences result from subtle differences in the regulation of their development. Altogether, the Triticeae global proteomes reported in this study, along with the first ever proteomic characterization of stigma development, constitute an invaluable resource to accelerate further studies of pollen and stigma development and interactions, a somewhat neglected area of research given their importance to plant and crop reproduction. 


\section{Chapter 4}

\section{General Discussion}

Plant sexual reproduction involves complex molecular interactions between the pistil and the pollen in order to facilitate the transport of the sperm cells to the ovary for fertilization. It commences when the mature stigma and pollen grain meet (Lord and Russell, 2002) and it might be expected that the protein complement of the mature stigma and pollen will reflect their readiness for their upcoming interactions. We therefore hypothesized that proteins immediately involved in pollen-pistil interactions are more likely to increase in abundance as the stigma reaches maturity and that the mature stigma and pollen grain would be enriched in proteins important for their impending encounter.

We tested this hypothesis using a gel-free shotgun proteomic approach in two species that not only can serve as representatives of important crops, but that also possess morphologically very different mature pollen and stigma, namely, B. carinata and triticale. To study differential abundance during stigma maturation, iTRAQ analysis was performed on both $B$. carinata and triticale stigma proteins extracted at developmental stages corresponding to their respective pollen BCP (early) and TCP (late) stages. A global proteomic analysis was also performed on the mature stigma and pollen grain from B. carinata and triticale using 2D HPLC-MS/MS.

In total, 7703 and 5608 B. carinata stigma and pollen proteins were identified respectively, and $251 B$. carinata stigma proteins were considered to be differentially abundant as the stigma matured, including 154 proteins that increased in abundance, and 97 proteins that decreased in abundance. For triticale, a total of 11533 and 2977 stigma and pollen proteins were identified respectively, while 647 stigma proteins were found to 
be differentially abundant, with 305 proteins having increased abundance, and 342 having decreased abundance. This study generated the largest pollen and stigma proteomic datasets within the Brassicaceae and the Triticeae to date, and represents the first developmental proteomic analysis of the stigma within the Brassicaceae and the Poaceae. The total number stigma proteins identified from each analyses and the total number of spectra, peptides, unique peptides and proteins obtained in this study overall, can be found in Tables S4.1 and S4.2 respectively.

The functional distribution of the $B$. carinata and triticale pollen or stigma proteins was compared and for the most part, their general biological processes were found to be quite similar (Figure 3.6-3.11). This may indicate that despite the morphological differences between the two species, there is still considerable functional convergence resulting from the fact that the stigmas of both species are dry, contain unicellular papillae, and their pollen is released at the tricellular stage (Heslop- Harrison and Shivanna, 1977). Nonetheless, in spite of their overall functional similarities, noticeable differences were observed. For example, the $B$. carinata stigma had a higher proportion of proteins in the Response to Stress and Defense categories than the triticale stigma (Figure 3.6). Furthermore, more proteins had increased abundance in these categories in the maturing B. carinata stigma than in triticale (Figure 3.10). This may reflect the fact that triticale is cleistogamous with the stigma being fully enclosed within the flower throughout its development. The triticale stigma would therefore not be as exposed as the exerted mature stigma of the chasmogamous B. carinata flower. Consequently, the $B$. carinata mature stigma could be anticipated to possess more proteins having roles in Response to Stress and Defense given that, unlike triticale, it 
would not have the floral protective physical barriers to biotic and abiotic stresses.

Another observable difference found between the stigmas of the two species was the fact that a significantly larger proportion of proteins involved in Protein Metabolic Process decreased in abundance in the maturing triticale stigma than in B. carinata (Figure 3.11). Although the reason for this difference is unclear, at least $40 \%$ of these proteins in triticale were involved in gene expression, while the few $B$. carinata stigma proteins with decreased protein levels were involved in a number of processes ranging from the proteolysis of propeptides of storage proteins, to transcription and hormone signaling to name a few (Table S2.7 and S3.7). Other differences existed between the types of proteins identified in the different proteomes. For example, the B. carinata stigma had a higher proportion of proteins involved in glucosinolate metabolism and a somewhat higher proportion of proteins involved in photosynthesis (Table S2.1 and S3.1). This was similar to a previous comparative study performed on $B$. napus and triticale stigmas (Nazemof et. al., 2016). The higher abundance of proteins involved in photosynthesis is reflected in the difference in pigmentation between the Brassica and triticale stigmas, and the higher proportion of glucosinolates is expected as these secondary plant metabolites are characteristic of the Brassicaceae (Alexander et. al., 2008). Conversely, the triticale stigma proteome contained several orthologs to FAR1 and FAR1 related TFs (Table S3.1), while the B. carinata stigma proteome contained none (Table S2.1). As mentioned above, these proteins regulate developmental processes in response to red and far-red light (Siddiqui et. al., 2016). Far-red radiation regulates phytochrome-mediated morphological and developmental plant responses to promote radiation capture and 
survival under shade (Park and Runkle, 2017), which may explain their presence in the stigma of the cleistogamous triticale flower.

Like the stigma proteins, despite their similarity in overall functional distribution, there were a few differences between the kinds of proteins identified in the B. carinata and triticale pollen proteomes and the reasons for many of these differences are unknown. However, several members of the oleosin family, including the tapetal oleosins, were identified in B. carinata pollen whereas none were identified in triticale (Table S2.1 and S3.1). Tapetal oleosins are distinct to the Brassicaceae family (Rejón et. al., 2016).

The mature pollen or stigma proteomes of B. carinata and triticale evidently share considerable functional features. Likewise, when comparing the pollen proteomes to that of the stigmas within the two species, similarities were also observed and this is in line with the fact that common processes like pollen germination and tube growth need to be supported by both the pollen and stigma. In light of this, the functional differences between their respective mature stigma and pollen proteomes will be discussed in general.

When the pollen grain is released from the anthers, it is released in a partially desiccated dormant state (Grobei, et. al., 2009). The stigma however, remains metabolically active as it approaches maturity. This is reflected in the types of biological processes that were enriched and under-represented in the pollen and stigma. For example, there were more unique biological processes that pertained to the Cell Cycle, Chromosome Organization, Gene Expression, Photosynthesis, Protein Metabolism, Ribonucleoprotein Complex Biogenesis and Organization, and RNA Metabolism that were enriched in the stigma than the pollen (Figure 2.4 and 3.4). Furthermore, the stigma 
also contained significantly more uniquely enriched processes involved in Response to Stimulus and Defense Response than the pollen, and in fact, all the processes involved in the Defense Response were under-represented in the B. carinata pollen (Figure 2.4 and 3.4). This is in agreement with the fact that the receptive stigma needs to be responsive to many different external stimuli in addition to producing many proteins involved in defense for protection against invasive microorganisms that could take advantage of the wound caused by the invading pollen tube. On the other hand, the pollen's highly resistant exine wall acts as a physical and chemical barrier to microbial and insect degradation (Edlund, et. al., 2017) and therefore might not need to have as many proteins readily available for defense or responses to stimuli.

One of the main categories for both the $B$. carinata and triticale pollen that contained a large number of enriched processes was Transport (Figure 2.3 and 3.3) with the pollen containing a significantly larger amount of uniquely enriched processes than the stigma (Figure 2.4 and 3.4). This is in line with transport roles during pollen tube growth. Vesicular Transport was particularly enriched in the pollen and is required for the transport of cell wall modifying enzymes and polysaccharides to the growing tube apex, while exocytosis at the tip and endocytosis at the subapical region is crucial for driving rapid polarized tip growth at the apex (Cheung and $\mathrm{Wu}, 2008)$. Conversely, transport within the stigma mostly facilitates pollen hydration, germination and tube growth by the provision of water, nutrients, enzymes and cell wall materials to the pollen contact site (Goring et. al., 2017).

Development was another category in which the differences between the stigma and the pollen were highlighted. In both species, the stigma expressed proteins involved 
in epidermal cell growth and development (Table S2.4 and S3.4) compatible with the fact that the stigma papillae are epidermal cells (Lolle and Pruitt, 1999). For the pollen, many of the proteins involved in development had roles in pollen and pollen tube development, cell tip growth and root hair growth (Table S2.5 and S3.5). These functional differences highlight the specialization of these cells.

The iTRAQ analysis of the developing $B$. carinata and triticale stigmas revealed numerous proteins that differed in abundance as the stigma matured. Preliminary analysis of the RNA-Sequencing data obtained from the equivalent B. carinata stigma stages validated the iTRAQ data and showed that the majority of proteins with elevated and decreased levels had matching up and down-regulated transcripts. In both species, many stigma proteins presumed to play a role in pollen-stigma interactions, increased in protein abundance. Some of the relevant findings are summarized below.

More stigma proteins involved in Defense and Response to Stress had elevated protein levels in accordance with the exposure and responsiveness of the stigma. In addition, in both species, many more proteins involved in Carbohydrate Metabolism and Cell Wall Organization and Biogenesis increased in protein abundance, and there was a significant overlap between the proteins identified in both these processes. This is likely due to the fact that enzymatic modification of the stigmatic layers is required for pollen tube penetration and growth through the stigma (Chapman and Goring 2010). Furthermore, carbohydrate metabolism is the main source of energy for pollen tube growth (Hirsche et. al., 2017).

Additionally, many more proteins involved in transport had increased than decreased abundance in the stigmas of both species. Examination of the corresponding 
proteins revealed many proteins involved in water transport, ion transport, and proton transport (Table S2.6 and S3.6). During hydration, water flows from the stigma to the pollen (Lord and Russell, 2002) and proteins involved in water transport would have increased abundance to facilitate this process. Furthermore, ion transport is important for pollen hydration because it maintains the turgidity of cells (Rehman and Yun, 2006) and hydrogen ion transport protein levels would be expected to be elevated as the stigma matures since cytosolic $\mathrm{pH}$ regulates actin cytoskeleton dynamics important for vesicular transport (Cheung and $\mathrm{Wu}, 2008)$.

In summary, the functional similarities observed between the mature stigma or pollen proteomes of $B$. carinata and triticale was reflective of their shared reproductive roles. However, an in depth comparison between the proteomes of their respective mature stigmas and pollen grains revealed significant differences that are likely attributable to their specialization and showed that their proteomes were enriched in proteins required for their upcoming interactions. Furthermore, the differential analyses revealed an increased abundance of proteins likely to be immediately required for pollenstigma interactions.

\subsection{Future Directions}

The results obtained in this study could serve as the basis for future research in a number of different directions. For example, the MS/MS peak lists were searched against the Viridiplantae, Brassiceae and Triticeae databases, and therefore many of the proteins identified were not $B$. carinata or triticale proteins but orthologs from other species within those databases. The MS/MS peak lists obtained from this study could be 
searched against an in silico translated proteome from the newly acquired B. carinata and triticale stigma and pollen transcriptomes in order to identify the corresponding proteins within those species. This would improve the accuracy of further analyses and experiments in these species.

A large number of the proteins identified in this study were assigned important roles in pollen and stigma interactions or development using the experimental evidence available from Arabidopsis orthologs. In addition, many proteins identified, such as protein kinases and transcription factors, had no known roles in these cells. Confirmation of the roles of these proteins in B. carinata or triticale reproduction could be achieved using genetic knock out experiments like T-DNA insertion lines or the CRISPR/cas9 system, for example.

The information obtained from this research can be used as a resource and allow for the discovery of potential protein functional interactions and/or protein clusters. For example, illustrative protein-protein interaction networks were predicted using some of the proteins identified within the B. carinata stigma and pollen proteomes (Figure 4.14.3). Such networks can illustrate the complexity of biological processes such as pollen tube growth, while also allowing the visualization of putative protein-protein interactions in a subset of proteins (Figure 4.1). They can also permit the visualization of putative protein-protein interactions in the stigma (Figure 4.2) or the pollen (Figure 4.3) in less complex processes like pollen hydration. These networks are predicted using information from a variety of sources including databases that contain evidence from direct proteinprotein interaction experiments, manually curated databases of metabolic and signal transduction pathways, known protein complexes, and information from gene expression 


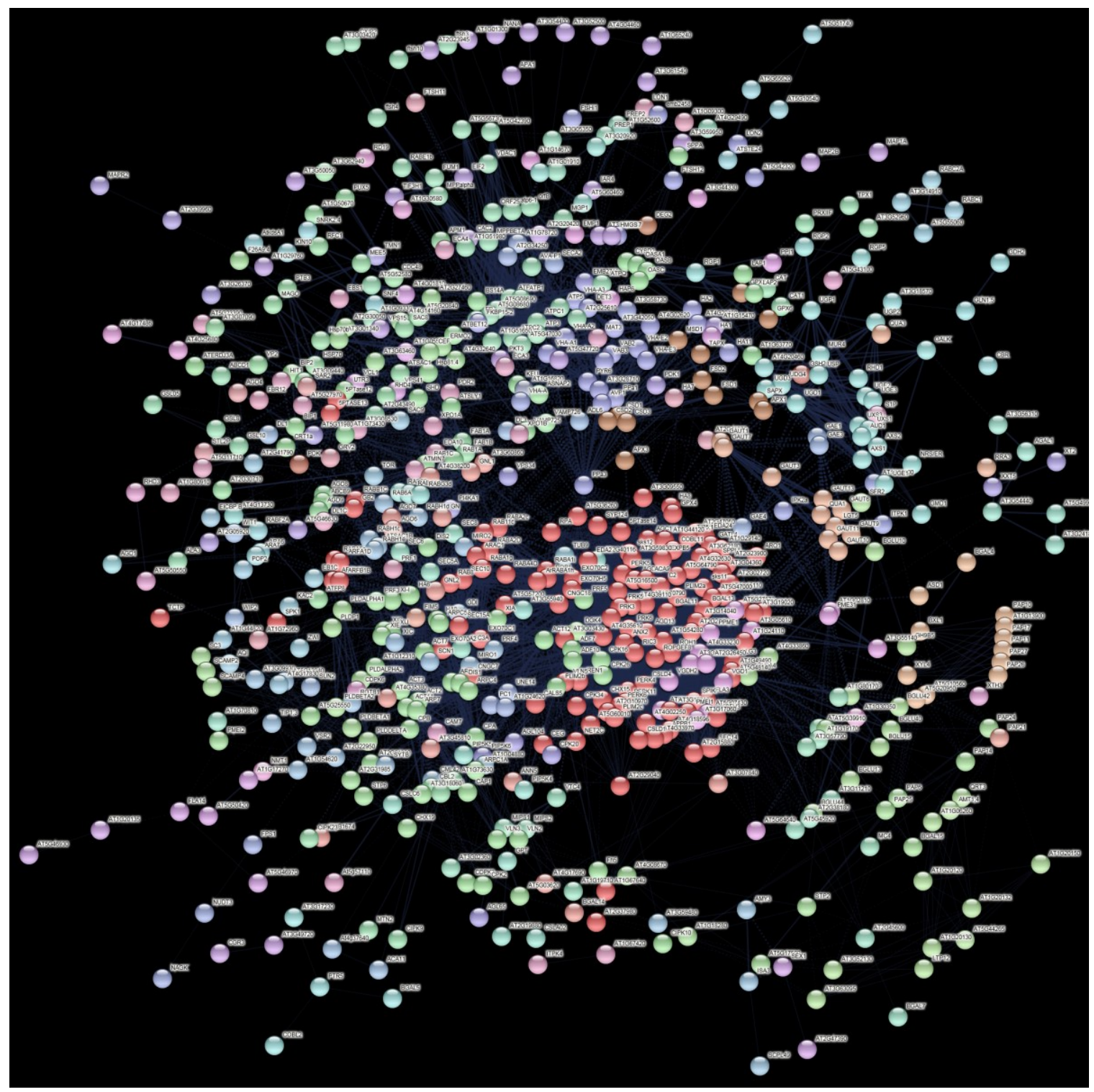

Figure 4.1. Network of putative $B$. carinata pollen protein-protein interactions involved in pollen tube growth. The grey edges represent putative interactions between proteins. The density of the edges represents the confidence of the putative functional associations. Colors correspond to clusters of proteins that putatively belong to the same protein complex or functional module. Solid lines represent interactions within a cluster, while dashed lines represent inter-cluster edges. 


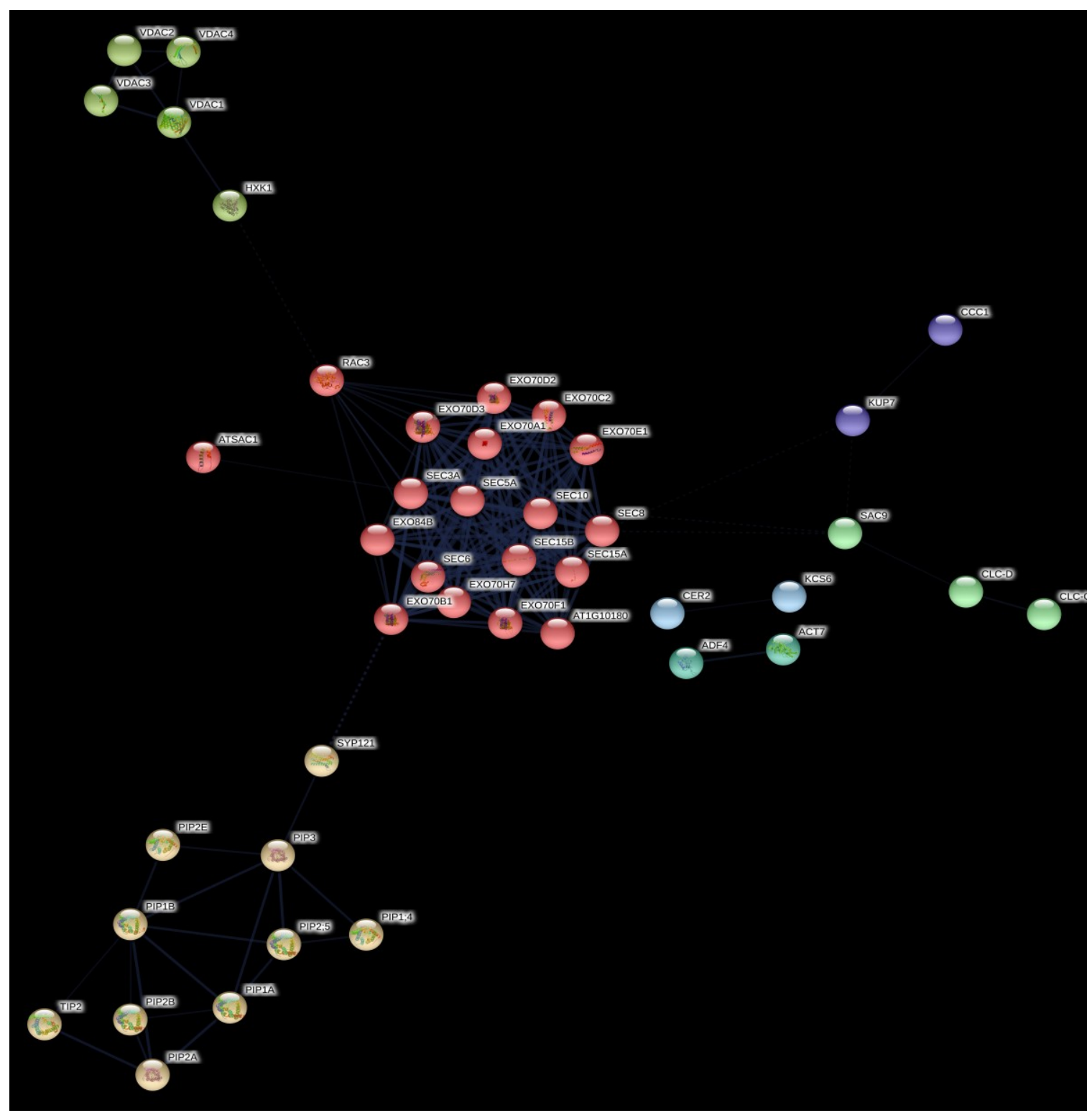

Figure 4.2. Network of putative $B$. carinata stigma protein-protein interactions involved in pollen hydration. The grey edges represent putative interactions between proteins. The density of the edges represents the confidence of putative functional associations. Colors correspond to clusters of proteins that putatively belong to the same protein complex or functional module. Solid lines represent interactions within a cluster, while dashed lines represent inter-cluster edges. 


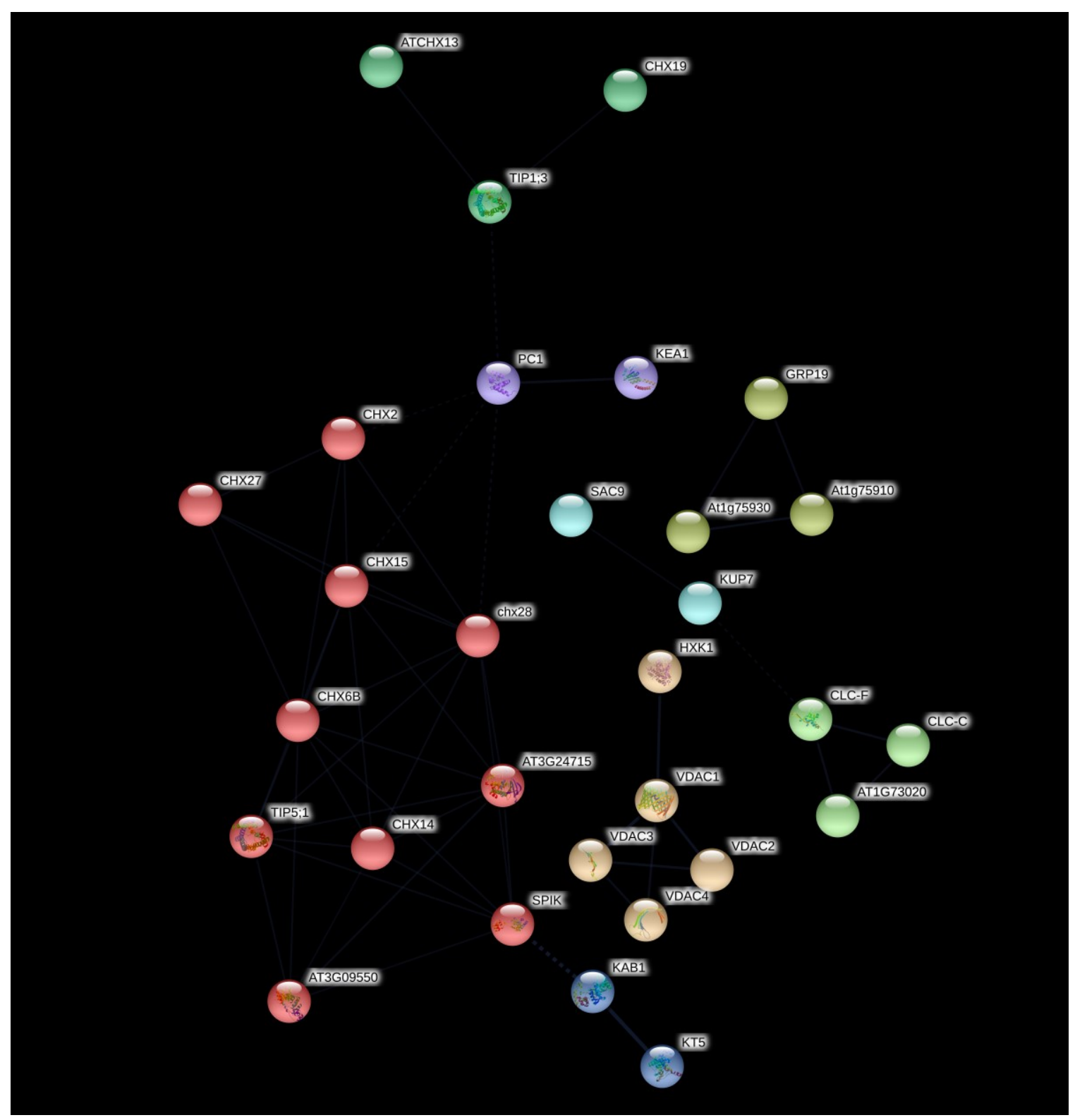

Figure 4.3. Network of putative $B$. carinata pollen protein-protein interactions involved in pollen hydration. The grey edges represent putative interactions between proteins. The density of the edges represents the confidence of putative functional associations. Colors correspond to clusters of proteins that putatively belong to the same protein complex or functional module. Solid lines represent interactions within a cluster, while dashed lines represent inter-cluster edges. 
experiments to name a few. The evidence for the protein-protein interactions can come from known information about the query proteins themselves. An example of this is the interaction between SYP121 (SYNTAXIN OF PLANTS121) and PIP3 (aquaporin PIP2;7) shown in Figure 4.2. Evidence for this interaction was taken from previous studies in which they were shown to physically interact using pull-down, biomolecular fluorescence complementation, and split ubiquitin assays (Hachez et. al., 2014). Conversely, evidence for the interactions within these functional interaction models are more often obtained from their orthologs in other species, as in the case of PC1 (pollen calcium-binding protein 1) and KEA1 ( $\mathrm{K}^{+}$efflux antiporter 1$)$ shown as a putative interaction in Figure 4.3. Orthologs of these proteins within Mus musculus were shown to interact using a co-sedimentation assay (Kislinger et. al., 2006). Many of these predicted interactions are therefore putative (Szklarczyk et. al., 2017). Predicted interactions can then be experimentally validated using protein-protein interaction assays like co-purification, co-crystallization, BioID or yeast two-hybrid experiments to name a few. For example, Brandão et. al. (2009) used an Arabidopsis protein interaction network to predict 45 putative interactions with a glutamyl-tRNA synthase. Three of the putative interactions were experimentally validated using yeast two-hybrid screening.

The differential abundance analysis performed in this study used the stigma tissue corresponding to the $\mathrm{BCP}$ and TCP stages in development. It would be interesting to examine the proteins that are differentially abundant at those particular developmental stages in the pollen. Additionally, these two stages were somewhat close in development, for example, there were no proteins that were absent from one stage and present in the other. Performing iTRAQ analysis using an earlier stage in stigma 
development could be pursued in the future. Such an experiment would also serve to confirm the trends in protein abundance observed in this study.

Due to the complexity of biological proteomes and the limitations of analytical separation methods, there is currently no single method that can provide qualitative and quantitative information about all the protein components within a complex biological sample (Abdallah et. al., 2012). Protein abundances within a biological sample can span over seven orders of magnitude. However, conventional proteomic approaches tend to be biased towards highly abundant proteins and will therefore only detect proteins within four orders of magnitude of the dynamic range, often excluding some of the more lower abundant proteins in the sample (Zubarev et. al., 2013). Consequently, each method provides a snapshot of the major protein constituents. Nonetheless, there are several approaches to expanding the proteome coverage. For example, a longer time spent on the separation column has been reported to increase the proteome depth. Additionally, more extensive fractionation procedures (for example 3D fractionation instead of 2D) may assist in reducing sample complexity for deeper exploration of the proteome.

Furthermore, the usage of hexapeptide libraries and diffusion based trypsin approaches to selectively deplete abundant proteins will also assist in the identification of those lower abundant proteins, therefore expanding proteome coverage (Zubarev et. al., 2013). Moreover, the sensitivity of mass spectrometers is ever increasing. New data independent acquisition approaches to shotgun proteomics such as $\mathrm{MS}^{\mathrm{E}}$ or SWATH MS allow for the fragmentation of all precursor ions that elute from the column (instead of the selection of more abundant ions used in more conventional approaches) and can therefore offer a higher dynamic range of proteins identified (Hu et. al., 2015). 


\subsection{General Conclusion}

The pollen-stigma interaction is essentially an interaction between two cells, the pollen vegetative cell and the stigma papillar cell that are suddenly and intentionally brought together from two different parts of the plant. These cells meet for the first time at a late stage in their maturation process and would likely remain in this state of preparation if kept apart. However, once they make contact, a whole gamut of molecular events is triggered that leads to profound and often unique changes in their biochemistry and morphology. This study provides the most in-depth coverage of the thousands of proteins these events rely upon, and often reveals the unsuspected presence of known proteins such as transcription factors and protein kinases. Enrichment and differential abundance analyses pointed to prevalent processes within the pollen-stigma interactions and identified numerous candidate proteins for specific roles within these processes that could be the subject of further research. Furthermore, a comparison of B. carinata and triticale stigma or pollen proteomes revealed a conservation of biological processes across taxa. 


\section{References}

1. Abe, M.; Katsumata, H.; Komeda, Y.; Takahashi, T. Regulation of shoot epidermal cell differentiation by a pair of homeodomain proteins in Arabidopsis. Development, 2003, $130,635-643$.

2. Abdallah, C.; Dumas-Gaudot, E.; Renaut, J.; Sergeant, K. Gel-based and gel-free quantitative proteomics approaches at a glance. Int. J. Plant Genomics. 2012, 2012, 1-17.

3. Aguilar, MI. HPLC of peptides and proteins: Methods and protocols; Springer: Berlin, Germany, 2004; pp 2-102.

4. Alemayehu, N.; Becker, H. Genotypic diversity and patterns of variation in a germplasm material of Ethiopian mustard (Brassica carinata A. Braun). Genet. Resour. Crop Evol. $2002,49,573-582$.

5. Alexander, J.; Auounsson, G. A.; Benford, D.; Cockburn, A.; Cravedi, J. P.; Dogliotti, E.; Di Domenico, A.; Férnandez-Cruz, M. L.; Fürst, P.; Fink-Gremmels, J.; Galli, C. L.; Grandjean, P.; Gzyl, J.; Heinemeyer, G.; Johansson, N.; Mutti, A.; Schlatter, J.; van Leeuwen, R.; Van Peteghem, C.; Verger, P. Glucosinolates as undesirable substances in animal feed: Scientific Panel on Contaminants in the Food Chain. EFSA J. 2008, 590, 176.

6. Alvarez, J. P.; Goldshmidt, A.; Efroni, I.; Bowman, J. L.; Eshed, Y. The NGATHA distal organ development genes are essential for style specification in Arabidopsis. Plant Cell. 2009, 21, 1373-1393. 
7. Alvarez, J.; Smyth, D. R. CRABS CLAW and SPATULA, two Arabidopsis genes that control carpel development in parallel with AGAMOUS. Development. 1999, 126, 2377 2386.

8. Atmodjo, M. A.; Sakuragi, Y.; Zhu, X.; Burrell, A. J.; Mohanty, S. S.; Atwood, J. A. $3^{\text {rd }}$; Orlando, R.; Scheller, H. V.; Mohnen, D. Galacturonosyltransferase (GAUT)1 and GAUT7 are the core of a plant cell wall pectin biosynthetic homogalacturonan:galacturonosyltransferase complex. PNAS. USA. 2011, 108, 2022520230.

9. Baggerman, G.; Vierstraete, E.; De Loof, A.; Schoofs, L. Gel-based versus gel-free proteomics: A review. Comb. Chem. High Throughput Screening. 2005, 8, 669-677.

10. Ballester, P.; Navarrete-Gómez, M.; Carbonero, P.; Oñate-Sánchez, L.; Ferrándiz, C. Leaf expansion in Arabidopsis is controlled by a TCP-NGA regulatory module likely conserved in distantly related species. Plant Physiol. 2015, 155, 21-32.

11. Barre, R. S. Interaction between Scrambled and Inflorescence Meristem Receptor Like Kinase 2 in inflorescence stem growth and root epidermal patterning in Arabidopsis thaliana. M.Sc. Thesis, Long Island University, Richard L. Conolly College, NY, September 2016 .

12. Barro, F.; Fernández-Escobar, J.; De La Vega, M.; Martin, A. Doubled haploid lines of Brassica carinata with modified erucic acid content through mutagenesis by EMS treatment of isolated microspores. Plant Breed. 2000, 120, 262-264.

13. Baum, B.; Gupta, P. K. Taxonomic examination of Triticale (x Triticosecale). Can. J. Bot. 1990, 68, 1889-1893. 
14. Baumann, U.; Juttner, J.; Bian, X.; Langridge, P. Self-incompatibility in the grasses. Ann. Bot. 2000, 85, 203-209.

15. Benghezal, M.; Cornillon, S.; Gebbie, L.; Alibaud, L.; Brückert, F.; Letourneur, F.; Cosson, P. Synergistic control of cellular adhesion by transmembrane 9 proteins. Mol. Biol. Cell. 2003, 14, 2890-2899.

16. Berkowitz, O.; Jost, R.; Pollmann, S.; Masle, J. Characterization of TCTP, the translationally controlled tumor protein, from Arabidopsis thaliana. Plant Cell. 2008, 20, $3430-3447$.

17. Blackmore, S.; Knox, R. B. Microspores. Evolution and Ontogeny; Academic Press Limited: London, UK, 1990; pp 173-242.

18. Boavida, L. C.; Vieira, A. M.; Becker, J. D.; Feijó, J. A. Gametophyte interaction and sexual reproduction: how plants make a zygote. Int. J. Dev. Biol. 2005, 49, 615-632.

19. Boisson-Dernier, A.; Kessler, S. A.; Grossniklaus, U. The walls have ears: the role of plant CrRLK1Ls in sensing and transducing extracellular signals. J. Exp. Bot. 2011, 62, $1581-1591$.

20. Boisson-Dernier, A.; Lituiev, D. S.; Nestorova, A.; Franck, C. M.; Thirugnanarajah, S.; Grossniklaus, U. ANXUR receptor-like kinases coordinate cell wall integrity with growth at the pollen tube tip via NADPH oxidases. PLoS Biol. 2013, 11, e1001719.

21. Borowska-Wykręt, D.; Elsner, J.; De Veylder, L.; Kwiatkowska, D. Defects in leaf epidermis of Arabidopsis thaliana plants with CDKA;1 activity reduced in the shoot apical meristem. Protoplasma. 2013, 250, 955-961.

22. Bosch, M.; Helper, P. K. Pectin methylesterases and pectin dynamics in pollen tubes. Plant Cell. 2005, 17, 3219-3226. 
23. Boudsocq, M.; Willmann, M. R.; McCormack, M.; Lee, H.; Shan, L.; He, P.; Bush, J.; Cheng, S.; Sheen, J. Differential innate immune signaling via $\mathrm{Ca}^{2+}$ sensor protein kinases. Nature, 2010, 464, 418-422.

24. Brandão, M. M.; Dantas, L. L; Silva-Filho, M. C. AtPIN: Arabidopsis thaliana protein interaction network. BMC Bioinformatics. 2009, DOI: 10.1186/1471-2105-10-454.

25. Buszewski, B.; Noga, S. Hydrophilic interaction liquid chromatography (HILIC) - a powerful separation technique. Anal. Bioanal. Chem. 2012, 402, 231-247.

26. Cai, G.; Parrotta, L.; Cresti, M. Organelle trafficking, the cytoskeleton, and pollen tube growth. J. Integr. Plant Biol. 2015, 57, 63-78.

27. Cai, H.; Zhao, L.; Wang, L.; Zhang, M.; Su, Z.; Cheng, Y.; Zhao, H.; Qin, Y. ERECTA signaling controls Arabidopsis inflorescence architecture through chromatin-mediated activation of PRE1 expression. New Phytol. 2017, 214, 1579-1596.

28. Carvalho, A.; Matos, M.; Lima-Brito, J.; Guedes-Pinto, H.; Benito, C. DNA fingerprint of F1 interspecific hybrids from the Triticeae tribe using ISSRs. Euphytica. 2005, 143, 93-99.

29. Chaiwongsar, S.; Strohm, A. K.; Su, S.; Krysan, P. J. Genetic analysis of the Arabidopsis protein kinases $M A P 3 K \varepsilon 1$ and $M A P 3 K \varepsilon 2$ indicates roles in cell expansion and embryo development. Front. Plant Sci. 2012, 3, 1-10.

30. Chandler, J. W. Auxin response factors. Plant, Cell Environ. 2015, DOI: https://doi.org/10.1111/pce.12662.

31. Chang, H.; Zhang, C.; Chang, Y.; Zhu, J.; Xu, X.; Shi, Z.; Zhang, X.; Xu, L.; Huang, H.; Zhang, S.; Yang, Z. NO PRIMEXINE AND PLASMA MEMBRANE UNDULATION is 
essential for primexine deposition and plasma membrane undulation during microsporogenesis in Arabidopsis. Plant Physiol. 2012, 158, 264-272.

32. Chao, Q.; Gao, Z.; Wang, Y.; Li, Z.; Huang, X.; Wang, Y.; Mei, Y.; Zhao, B.; Li, L.; Jiang, Y.; Wang, B. The proteome and phosphoproteome of maize pollen uncovers fertility candidate proteins. Plant. Mol. Biol. 2016, 91, 287-304.

33. Chapman, B. D.; Salmon, C. D.; Dyson, D.; Blackley, K. Triticale production and utilization manual. Spring and winter Triticale for grain, forage and value-added. Alberta Agriculture, Food and Rural Development: Edmonton, AB, 2005; https://www1.agric.gov.ab.ca/\$department/deptdocs.nsf/all/fcd5464/\$FILE/2005triticale manual.pdf

34. Chapman, L. A.; Goring, D. R. Pollen-pistil interactions regulating successful fertilization in the Brassicaceae. J. Exp. Bot. 2010, 61, 1987-1999.

35. Chapman, L. A.; Goring, D. R. Misregulation of phosphoinositides in Arabidopsis thaliana decreases pollen hydration and maternal fertility. Sex. Plant Reprod. 2011, 24, 319-326.

36. Chen, C.; Wang, S.; Huang, H. LEUNIG has multiple functions in gynoecium development in Arabidopsis. Genesis. 2000, 26, 42-54.

37. Chen. J.; Yang, Z. Novel ABP1-TMK auxin sensing system controls ROP GTPasemediated interdigitated cell expansion in Arabidopsis. Small GTPases. 2014, e29711.

38. Chen, Y.; Zou, M.; Cao, Y. Transcriptome analysis of the Arabidopsis semi-in vivo pollen tube guidance system uncovers a distinct gene expression profile. J. Plant Biol. 2014, 57, 93-105. 
39. Cheung, A. Y.; Wu, H. Structural and signaling networks for the polar cell growth machinery in pollen tubes. Annu. Rev. Plant Biol. 2008, 59, 547-572.

40. Colcombet, J.; Sözen, C.; Hirt, H. Convergence of multiple MAP3Ks on MKK3 identifies as set of novel stress MAPK modules. Front. Plant Sci. 2016, DOI: 10.3389/fpls.2016.01941.

41. Cole, R. A.; Synek, L.; Zarsky, V.; Fowler, J. E. SEC8, a subunit of the putative Arabidopsis exocyst complex, facilitates pollen germination and competitive pollen tube growth. Plant Physiol. 2005, 138, 2005-2018.

42. Craddock, C. P.; Adams, N.; Kroon, J. T.; Bryant, F. M.; Hussey, P. J.; Kurup, S.; Eastmond, P. J. Cyclin-dependent kinase activity enhances phosphatidylcholine biosynthesis in Arabidopsis by repressing phosphatidic acid phosphohydrolase activity. Plant J. 2017, 89, 3-14.

43. Crawford, B. C. W.; Yanofsky, M. F. HALF FILLED promotes reproductive tract development and fertilization efficiency in Arabidopsis thaliana. Development. 2011, 138, 2999-3009.

44. Cresti, M.; Tiezzi, A. Sexual plant reproduction; Springer: Berlin, Germany, 2011; pp 6087.

45. Cui, X.; Fan, B.; Scholz, J.; Chen, Z. Roles of Arabidopsis cyclin-dependent kinase C complexes in cauliflower mosaic virus infection, plant growth, and development. Plant Cell. 2007, 19, 1388-1402.

46. Da Costa, M. L.; Pereira, L. G.; Coimbra, S. Growth media induces variation in cell wall associated gene expression in Arabidopsis thaliana pollen tube. Plants. 2013, 2, 429-440. 
47. Dai, S.; Chen, T.; Chong, K.; Xue, Y.; Liu, S.; Wang, T. Proteomics identification of differentially expressed proteins associated with pollen germination and tube growth reveals characteristics of germinated Oryza sativa pollen. Mol. Cell. Proteomics 2007, 6, $207-230$.

48. Dai, S.; Li, L.; Chen, T.; Chong, K.; Xue, Y.; Wang, T. Proteomic analyses of Oryza sativa mature pollen reveal novel proteins associated with pollen germination and tube growth. Proteomics 2006, 6, 2504-2529.

49. Darke, R. The encyclopedia of grasses for livable landscapes. Timber Press: Portland, Oregon, USA, 2007; pp 40.

50. Datta, S. K. Androgenic haploids: Factors controlling development and its application in crop improvement. Curr. Sci. 2005, 89, 1870-1878.

51. Davis, A. R.; Fowke, L. C.; Sawhney, V. K.; Low, N. H. Floral nectar secretion and ploidy in Brassica rapa and B. napus (Brassicaceae). II. Quantified variability of nectary structure and function in rapid-cycling lines. Ann. Bot. 1996, 77, 223-234.

52. Deepak, S.; Shailasree, S.; Kini, R. K.; Muck, A.; Mithöfer, A.; Shetty, S. H. Hydroxyproline-rich glycoproteins and plant defence. J. Phytopathol. 2010, 158, 585593.

53. Delmas, F.; Séveno, M.; Northey, J. G. B.; Hernould, M.; Lerouge, P.; McCourt, P.; Chevalier, C. The synthesis of the rhamnogalacturonan II component 3-deoxy-D-manno2-octulosonic acid (Kdo) is required for pollen tube growth and elongation. J. Exp. Bot. 2008, 59, 2639-2647.

54. Dixit, R.; Nasrallah, J. B. Recognizing self in the self-incompatibility response. Plant Physiol. 2001, 125, 105-108. 
55. Dong, J.; Kim, S. T.; Lord E. M. Plantacyanin plays a role in reproduction in Arabidopsis. Plant Physiol. 2005, 138, 778-789.

56. Dong, X.; Hong, Z.; Sivaramakrishnan, M.; Mahfouz, M.; Verma, D. P. S. Callose synthase (CalS5) is required for exine formation during microgametogenesis and for pollen viability in Arabidopsis. Plant J. 2005, 42, 315-328.

57. Dornelas, M. C.; Van Lammeren, A. A. M.; Kreis, M. Arabidopsis thaliana SHAGGYrelated protein kinases (AtSK11 and 12) function in perianth and gynoecium development. Plant J. 2000, 21, 419-429.

58. Doucet, J.; Lee, H. K.; Goring, D. R. Pollen acceptance or rejection: A tale of two pathways. Trends Plant Sci. 2016, 21, 1058-1067.

59. Dranginis, A. M.; Rauceo, J. M.; Coronado, J. E.; Lipke, P. N. A biochemical guide to yeast adhesins: glycoproteins for social and antisocial occasions. Microbiol. Mol. Biol. Rev. 2007, 71, 282-294.

60. Dresselhaus, T.; Franklin-Tong, N. Male-female crosstalk during pollen germination, tube growth and guidance, and double fertilization. Mol. Plant. 2013, 6, 1018-1036.

61. Dresselhaus, T.; Lausser, A.; Márton, M. L. Using maize as a model to study pollen tube growth and guidance, cross-incompatibility and sperm delivery in grasses. Ann. Bot. $2011,108,727-737$.

62. East, M. P.; Kahn, R. A. Models for the functions of Arf GAPs. Semin. Cell Dev. Biol. 2011, 22, 3-9.

63. Ebine, K.; Inoue, T.; Ito, J.; Ito, E.; Uemura, T.; Goh, T.; Abe, H.; Sato, K.; Nakano, A.; Ueda, T. Plant vacuolar trafficking occurs through distinctly regulated pathways. Curr. Biol. 2014, 24, 1375-1382. 
64. Edlund, A. F.; Olsen, K.; Mendoza, C.; Wang, J.; Buckley, T.; Nguyen, M.; Callahan, B.; Owen, H. A. Pollen wall degradation in the Brassicaceae permits cell emergence after pollination. Am. J. Bot. 2017, 104, 1266-1273.

65. Edlund, A. F.; Swanson, R.; Preuss, D. Pollen and stigma structure and function: The role of diversity in pollination. Plant Cell. 2004, 16, S84- S97.

66. Eisenach, C.; Chen, Z.; Grefen, C.; Blatt, M. R. The trafficking protein SYP121 of Arabidopsis connects programmed stomatal closure and $\mathrm{K}^{+}$channel activity with vegetative growth. Plant J. 2012, 69, 241-251.

67. Eshed, Y.; Baum, S. F.; Bowman, J. L. Distinct mechanisms promote polarity establishment in carpels of Arabidopsis. Cell. 1999, 99, 199-209.

68. Espinosa-Ruiz, A.; Martínez, C.; de Lucas, M.; Fàbregas, N.; Bosch, N.; Caño-Delgado, A.; Prat, S. TOPLESS mediates brassinosteroid control of shoot boundaries and root meristem development in Arabidopsis thaliana. Development. 2017, 144, 1619-1628.

69. Falbel, T. G.; Koch, L. M.; Nadeau, J. A.; Segui-Simarro, J. M.; Sack, F. D.; Bednarek, S. Y. SCD1 is required for cell cytokinesis and polarized cell expansion in Arabidopsis thaliana. Development. 2003, 130, 4011-4024.

70. Fan, X.; Yang, C.; Klisch, D.; Ferguson, A.; Bhaellero, R. P.; Niu, X.; Wilson, Z. A. ECHIDNA protein impacts on male fertility in Arabidopsis by mediating trans-Golgi network secretory trafficking during anther and pollen development. Plant Physiol. 2014, $164,1338-1349$.

71. Favaro, R.; Pinyopich, A.; Battaglia, R.; Kooiker, M.; Borghi, L.; Ditta, G.; Yanofsky, M. F.; Kater, M. M.; Colombo, L. MADS-Box protein complexes control carpel and ovule development in Arabidopsis. Plant Cell. 2003, 15, 2603-2611. 
72. Ferdous, A.; Kodadek, T.; Johnston, S. A. A nonproteolytic function of the 19S regulatory subunit of the $26 \mathrm{~S}$ proteasome is required for efficient activated transcription by human RNA polymerase II. Biochemistry. 2002, 41, 12798-12805.

73. Franks, R. G.; Wang, C.; Levin, J. Z.; Liu, Z. SEUSS, a member of a novel family of plant regulatory proteins, represses floral homeotic gene expression with $L E U N I G$. Development. 2002, 129, 253-263.

74. Friedman, J.; Barrett, S. Wind of change: new insights on the ecology and evolution of pollination and mating in wind-pollinated plants. Ann. Bot. 2009, 103, 1515-1527.

75. Fujimoto, M.; Tsutsumi, N. Dynamin-related proteins in plant post-Golgi traffic. Front. Plant Sci. 2014, 5, 408.

76. Furumizu, C.; Alvarez, J. P.; Sakakibara, K.; Bowman, J. L. Antagonistic roles for KNOX1 and KNOX2 genes in patterning the land plant body plan following an ancient gene duplication. PLoS Genet. 2015, 11, e1004980.

77. Galindo-Trigo, S.; Gray, J. E.; Smith, L. M. Conserved roles of CrRLK1L receptor-like kinases in cell expansion and reproduction from algae to angiosperms. Front. Plant Sci. 2016, DOI: $10.3389 /$ fpls.2016.01269.

78. Gangappa, S. N.; Botto, J.F. The BBX family of plant transcription factors. Trends Plant Sci. 2014, 19, 460-470.

79. Gao, X.; Liu, C. Z.; Li, D. D.; Zhao, T. T.; Li, F.; Xiao, N. J.; Zhao, X.; Zhang, X. S. The Arabidopsis KIN $\beta \gamma$ subunit of the SnRK1 complex regulates pollen hydration on the stigma by mediating the level of reactive oxygen species in pollen. PLoS Genet. 2016, 12, e1006228. 
80. Gaude, T.; Dumas, C. Organization of stigma surface components in Brassica: A cytochemical study. J. Cell Sci. 1986, 82, 203-216.

81. Getinet, A.; Rakow, G.; Raney, J. P.; Downey, R. K. Development of zero erucic acid Ethiopian mustard through an interspecific cross with zero erucic acid Oriental mustard. Can. J. Plant Sci. 1994, 74, 793-795.

82. Gilroy, S.; Suzuki, N.; Miller, G.; Choi, W.; Toyota, M.; Devireddy, A. R.; Mittler, R. A tidal wave of signals: calcium and ROS at the forefront of rapid systemic signaling. Trends Plant Sci. 2014, 19, 623-630.

83. Goetz, M.; Vivian-Smith, A.; Johnson, S. D.; Koltunow, A. M. AUXIN RESPONSE FACTOR8 is a negative regulator of fruit initiation in Arabidopsis. Plant Cell. 2006, 18, 1873-1886.

84. Golovnina, K. A.; Glushkov, S. A.; Blinov, A. G.; Mayorov, V. I.; Adkison, L. R.; Goncharov, N. P. Molecular phylogeny of the genus Triticum L. Plant Syst. Evol. 2007, 264, 195-216.

85. Gonzalez, D. H., Ed. Plant transcription factors. Evolutionary, structural and functional aspects; Academic Press: London Wall, U. K., 2016; pp xi, 215-216.

86. Goring, D. R. Exocyst, exosomes, and autophagy in the regulation of Brassicaceae pollen-stigma interactions. J. Exp. Bot. 2017, 69, 69-78.

87. Grass Phylogeny Working Group; Barker, N. P.; Clark, L. G.; Davis, J. I.; Duvall, M. R.; Guala, G. F.; Hsiao, C.; Kellogg, E. A.; Linder, H. P.; Mason-Gamer, R. J.; Mathews, S. Y.; Simmons, M. P.; Soreng, R. J.; Spangler, R. E. Phylogeny and subfamilial classification of the Grasses (Poaceae). Ann. Mo. Bot. Gard. 2001, 88, 373-457. 
88. Grobei, M. A.; Qeli, E.; Brunner, E.; Rehrauer, H.; Zhang, R.; Roschitzki, B.; Basler, K.; Ahrens, C. H.; Grossniklaus, U. Deterministic protein inference for shotgun proteomics data provides new insights into Arabidopsis pollen development and function. Genome Res. 2009, 19, 1786-1800.

89. Guan, Y.; Lu, J.; Xu, J.; McClure, B.; Zhang, S. Two mitogen-activated protein kinases, MPK3 and MPK6, are required for funicular guidance of pollen tubes in Arabidopsis. Plant Physiol. 2014, 165, 528-533.

90. Guilfoyle, T. J.; Hagen, G. Auxin response factors. Curr. Opin. Plant Biol. 2007, 10, 453460.

91. Guyomarc'h, S.; Benhamed, M.; Lemonnier, G.; Renou, J.; Zhou, D.; Delarue, M. MGOUN3: evidence for chromatin-mediated regulation of FLC expression. J. Exp. Bot. 2006, 57, 2111-2119.

92. Hachez, C.; Laloux, T.; Reinhardt, H.; Cavez, D.; Degand, H.; Grefen, C.; De Rycke, R.; Inzé, D.; Blatt, M. R.; Russinova, E.; Chaumont, F. Arabidopsis SNAREs SYP61 and SYP121 coordinate the trafficking of plasma membrane aquaporin PIP2;7 to modulate the cell membrane water permeability. Plant Cell. 2014, 26, 3132-3147.

93. Hackett, R. M.; Cadwallader, G.; Franklin, F. C. Functional analysis of a Brassica oleracea SLR1 gene promoter. Plant Physiol. 1996, 112, 1601-1607.

94. Hála, M.; Cole, R.; Synek, L.; Drdová, E.; Pecenková, T.; Nordheim, A.; Lamkemeyer, T.; Madlung, J.; Hochholdinger, F.; Fowler, J. E.; Zárský, V. An exocyst complex functions in plant cell growth in Arabidopsis and tobacco. Plant Cell. 2008, 20, 13301045. 
95. Hamilton, E. S.; Haswell, E. S. The tension-sensitive ion transport activity of MSL8 is critical for its function in pollen hydration and germination. Plant Cell Physiol. 2017, 58, 1222-1237.

96. Hao, L.; Liu, J.; Zhong, S.; Gu, H.; Qu, Li. AtVPS41-mediated endocytic pathway is essential for pollen tube-stigma interaction in Arabidopsis. PNAS. 2016, 113, 6307-6312.

97. Haslam, T. M.; Mañas-Fernández, A.; Zhao, L.; Kunst, L. Arabidopsis ECERIFERUM2 is a component of the fatty acid elongation machinery required for fatty acid extension to exceptional lengths. Plant Physiol. 2012, 160, 1164-1174.

98. He, Z.; Zhao, X.; Kong, F.; Zuo, Z.; Liu, X. TCP2 positively regulates $H Y 5 / H Y H$ and photomorphogenesis in Arabidopsis. J. Exp. Bot. 2016, 67, 775-785.

99. Heard, W.; Sklenáŕ, J.; Tomé, D. F.; Robatzek, S.; Jones, A. M. Identification of regulatory and cargo proteins of endosomal and secretory pathways in Arabidopsis thaliana by proteomic dissection. Mol. Cell Proteomics. 2015, 14, 1796-1813.

100. Hegebarth, D.; Jetter R. Cuticular waxes of Arabidopsis thaliana shoots: celltype-specific composition and biosynthesis. Plants 2017, 6, 27.

101. Hemerly, A. S.; Ferreira, P.; de Almeida Engier, Janice.; Van Montagu, M.; Engler, G.; Inzé, D. $c d c 2 a$ expression in Arabidopsis is linked with competence for cell division. Plant Cell. 1993, 5, 1711-1723.

102. Heslop-Harrison, J.; Heslop-Harrison, Y. Enzymatic removal of the proteinaceous pellicle of the stigma papilla prevents pollen tube entry in the Caryophyllaceae. Ann. Bot. $1975,39,163-165$.

103. Heslop-Harrison, Y.; Shivanna, K. R. The receptive surface of the angiosperm stigma. Ann. Bot. 1977, 41, 1233-1258. 
104. Hicks, G. R.; Rojo, E.; Hong, S.; Carter, D. G.; Raikhel, N. V. Germinating pollen has tubular vacuoles, displays highly dynamic vacuole biogenesis, and requires VACUOLESS1 for proper function. Plant Physiol. 2004, 134, 1227-1239.

105. Hills, M. J.; Hall, L. M.; Messenger, D. F.; Graf, R. J.; Beres, B. L.; Eudes, F. Evaluation of crossability between triticale (X Triticosecale Wittmack) and common wheat; durum wheat and rye. Environ. Biosaf. Res. 2007, 6, 249-257.

106. Hirsche, J.; Fernández, J. M. G.; Stabentheiner, E.; Großkinsky, D. K.; Roitsch, T. Differential effects of carbohydrates on Arabidopsis pollen germination. Plant Cell Physiol. 2017, 58, 691-701.

107. Hiscock, S. J.; Allen, A. M. Diverse cell signalling pathways regulate pollenstigma interactions: the search for consensus. New Phytol. 2008, 179, 286-317.

108. Hiscock, S. J.; Brown, D.; Gurr, S. J.; Dickinson, H. G. Serine esterases are required for pollen tube penetration of the stigma in Brassica. Sex. Plant Reprod. 2002, $15,65-74$.

109. Hoedemaekers, K.; Derksen, J.; Hoogstrate, S. W.; Wolters-Arts, M.; Oh, S. A.; Twell, D.; Mariana, C.; Rieu, I. BURSTING POLLEN is required to organize the pollen germination plaque and pollen tube tip in Arabidopsis thaliana. New Phytol. 2015, 206, 255-267.

110. Holmes-Davis, R.; Tanaka, C. K.; Vensel, W. H.; Hurkman, W. J.; McCormick, S. Proteome mapping of mature pollen of Arabidopsis thaliana. Proteomics. 2005, 5, 4864-4884. 
111. $\mathrm{Hu}, \mathrm{J} . ;$ Rampitsch, C.; Bykova, N. V. Advances in plant proteomics toward improvement of crop productivity and stress resistance. Front. Plant Sci. 2015, DOI: 10.3389/fpls.2015.00209.

112. Hubbard, K. E.; Webb, A. A. R. Circadian rhythms in stomata: Physiological and molecular aspects. In Rhythms in plants; Mancuso, S.; Shabala, S. Eds.; Springer, Cham, Switzerland, 2015; pp 231-255.

113. Hwang, J.; Vernound, V.; Szumlanski, A.; Nielsen, E.; Yang, Z. A tip-localized Rho GTPase-activating protein controls cell polarity by globally inhibiting Rho GTPase at the cell apex. Curr. Biol. 2008, 18, 1907-1916.

114. Ichikawa, M.; Iwano, M.; Sato, M. H. Nuclear membrane localization during pollen development and apex-focused polarity establishment of SYP124/125 during pollen germination in Arabidopsis thaliana. Plant Reprod. 2015, 28, 143-151.

115. Idänheimo, N.; Gauthier, A.; Salojärvi, J.; Siligato, R.; Brosché, M.; Kollist, H.; Mähönen, A. P.; Kangasjärvi, J.; Wrzaczek, M. The Arabidopsis thaliana cysteinerich receptor-like kinases CRK6 and CRK7 protect against apoplastic oxidative stress. Biochem. Biophys. Res. Commun. 2014, 445, 457-462.

116. Jiang, L.; Yang, S. L.; Xie, L. F.; Puah, C. S.; Zhang, X. Q.; Yang, W. C.; Sundaresan, V.; Ye, D. VANGUARDI encodes a pectin methylesterase that enhances pollen tube growth in the Arabidopsis style and transmitting tract. Plant Cell. 2005, 17, 584-596.

117. Jimenez-Lopez, J. C.; Wang, X.; Kotchoni, S. O.; Huang, S.; Szymanski, D. B.; Staiger, C. J. Heterodimeric capping protein from Arabidopsis is a membrane-associated, actin-binding protein. Plant Physiol. 2014, 166, 1312-1328. 
118. Johnson, E. N. Carinata Production: A Guide to Best Management Practices. Agriculture and Agri-Food Canada: Saskatoon, SK, 2013; http://www.ndswcs.org/home_htm_files/Carinata_Production_Manual_080213.pdf.

119. Johnson-Brousseau, S. A.; McCormick, S. A compendium of methods useful for characterizing Arabidopsis pollen mutants and gametophytically-expressed genes. Plant J. 2004, 39, 761-775.

120. Jorrin-Novo, J. V.; Komatsu, S.; Weckwerth, W.; Wienkoop, S. Plant Proteomics: Methods and Protocols; Humana Press: Hertfordshire, UK, 2014; pp 10-146.

121. Jossier, M.; Kroniewicz, L.; Dalmas, F.; Thiec, D. L.; Ephritikhine, G.; Thomine, S.; Barbier-Brygoo, H.; Vavasseur, A.; Filleur, S.; Leonhardt. N. The Arabidopsis vacuolar anion transporter, AtCLCc, is involved in the regulation of stomatal movements and contributes to salt tolerance. Plant J. 2010, 64, 563-576.

122. Kamata, N.; Okada, H.; Komeda, Y.; Takahashi, T. Mutations in epidermisspecific HD-ZIP IV genes affect floral organ identity in Arabidopsis thaliana. Plant J. 2013, 75, 430-330.

123. Kandasamy, M. K.; Nasrallah, J. B.; Nasrallah, M. E. Pollen-pistil interactions and developmental regulation of pollen tube growth in Arabidopsis. Development. 1994, $120,3405-3418$.

124. Kang, B.; Busse, J. S.; Bednarek, S. Y. Members of the Arabidopsis dynamin-like gene family, ADL1 are essential for plant cytokinesis and polarized cell growth. Plant Cell. 2003, 15, 899-913.

125. Kapp, E.; Schutz, F. Overview of tandem mass spectrometry (MS/MS) database search algorithms. Curr. Protoc. Protein Sci. 2007, 25, 2.1-2.19. 
126. Kay, P.; Groszmann, M.; Ross, J. J.; Parish, R. W.; Swain, S. M. Modifications of a conserved regulatory network involving INDEHISCENT controls multiple aspects of reproductive tissue development in Arabidopsis. New Phytol. 2013, 197, 73-87.

127. Khosla, A.; Paper, J. M.; Boehler, A. P.; Bradley, A. M.; Neumann, T. R.; Schrick, K. HD-Zip proteins GL2 and HDG11 have redundant functions in Arabidopsis trichomes, and GL2 activates a positive feedback loop via MYB23. Plant Cell. 2014, 26, 2184-2200.

128. Kislinger, T.; Cox, B.; Kannan, A.; Chung, C.; Hu, P.; Ignatchenko, A.; Scott, M. S.; Gramolini, A. O.; Morris, Q.; Hallett, M. T.; Rossant, J.; Hughes, T. R.; Frey, B.; Emili, A. Global survey of organ and organelle protein expression in mouse: combined proteomic and transcriptomic profiling. Cell. 2006, 125, 173-186.

129. Kitashiba, H.; Nasrallah, J. B. Self-incompatibility in Brassicaceae crops: lessons for interspecific incompatibility. Breed. Sci. 2014, 64, 23-37.

130. Klaas, M.; Yang, B.; Bosch, M.; Thorogood, D.; Manzanares, C.; Armstead, I. P.; Franklin, F. C. H.; Barth, S. Progress towards elucidating the mechanisms of selfincompatibility in the grasses: further insights from studies in Lolium. Ann. Bot. 2011, $108,677-685$.

131. Konopka, C. A.; Bednarek, S. Y. Comparison of the dynamics and functional redundancy of the Arabidopsis dynamin-related isoforms DRP1A and DRP1C during plant development. Plant Physiol. 2008, 147, 1590-1602.

132. Kristal, A. R.; Lampe, J. W. Brassica vegetables and prostate cancer risk: A review of the epidemiological evidence. Nutr. Cancer. 2002, 42, 1-9. 
133. Kudo, G. Anther arrangement influences pollen deposition and removal in hermaphrodite flowers. Funct. Ecol. 2003, 17, 349-355.

134. Latrasse, D.; Germann, S.; Houba-Hérin, N.; Dubois, E.; Bui-Prodhomme, D.; Hourcade, D.; Juul-Jensen, T.; Le Roux, C.; Majira, A.; Simoncello, N.; Granier, F.; Taconnat, L.; Renou, J.; Gaudin, V. Control of flowering and cell fate by LIF2, an RNA binding partner of the polycomb complex component LHP1. PLoS One. 2011, 6, e16592.

135. Laurie, S.; Halford, N. G. The role of protein kinases in the regulation of plant growth and development. Plant Growth Regul. 2001, 34, 253-265.

136. Lelly, T.; Kazman, E.; Devos, K. M.; Gale, M. D. Use of RFLPs to determine the chromosome composition of the tetraploid triticale (A/B)(A/B)RR. Genome. 1995, 38, 250-254.

137. Li, S. The Arabidopsis thaliana TCP transcription factors: A broadening horizon beyond development. Plant Signal. Behav. 2015, 10, e1044192.

138. Li, D.; Guan, H.; Li, F., Liu, C.; Dong, Y.; Zhang, X.; Gao, X. Arabidopsis shaker pollen inward $\mathrm{K}^{+}$channel SPIK functions in SnRK1 complex-regulated pollen hydration on the stigma. J. Integr. Plant Biol. 2017, 59, 604-611.

139. Li, G.; Boudsocq, M.; Hem, S.; Vialaret, J.; Rossignol, M.; Maurel, C.; Santoni, V. The calcium-dependent protein kinase CPK7 acts on root hydraulic conductivity. Plant Cell Environ. 2015, 38, 1312-1320.

140. Li, H.; Chen, H.; Yang, Z.; Gong, J. Cdi gene is required for pollen germination and tube growth in Arabidopsis. FEBS Lett. 2012, 586, 1028-1031. 
141. Li, S.; M. A. van Os, G.; Ren, S.; Yu, D.; Ketelaar, T.; Emons, A. M. C.; Liu, C. Expression and functional analyses of EXO70 genes in Arabidopsis implicate their roles in regulating cell type-specific exocytosis. Plant Physiol. 2010, 154, 1819-1830.

142. Li, S.; Xie, Z.; Hu, C.; Zhang, J. A review of Auxin Response Factors (ARFs) in plants. Front. Plant Sci. 2016, DOI: 10.3389/fpls.2016.00047.

143. Li-Beisson, Y.; Pollard, M.; Sauveplane, V.; Pinot, F.; Ohlrogge, J.; Beisson, F. Nanoridges that characterize the surface morphology of flowers require the synthesis of cutin polyester. PNAS. 2009, 106, 22008-22013.

144. Liao, H.; Zhu, M.; Cui, H.; Du, X.; Tang, Y.; Chen, L.; Ye, D.; Zhang, X. MARIS plays important roles in Arabidopsis pollen tube and root hair growth. J. Integr. Plant Biol. 2016, 58, 927-940

145. Lin, J. S.; Huang, X. X.; Li, Q.; Cao, Y.; Bao, Y.; Meng, X. F.; Li, Y. J.; Fu. C.; Hou, B. K. UDP-glycosyltransferase 72B1 catalyzes the glucose conjugation of monolignols and is essential for the normal cell wall lignification in Arabidopsis thaliana. Plant J. 2016, 88, 26-42.

146. Lin, R.; Wang, H. Arabidopsis FHY3/FAR1 gene family and distinct roles of its members in light control of Arabidopsis development. Plant Physiol. 2004, 136, 40104022.

147. Liu, L.; Jiang, Y.; Zhang, X.; Wang, X.; Wang, Y.; Han, Y.; Coupland, G.; Jin, J. B.; Searle, I. R.; Fu, Y.; Chen, F. Two SUMO proteases SUMO PROTEASE RELATED TO FERTILITY 1 and -2 are required for fertility. Plant Physiol. 2017, 175, 1703-1719. 
148. Liu, C.; Li, L.; Chen, W.; Chen, X.; Xu, Z.; Bai, S. HDA18 affects cell fate in Arabidopsis root epidermis via histone acetylation at four kinase genes. Plant Cell. 2013, $25,257-269$.

149. Liu, C.; Yan, B.; Song, Y.; Xu, Y.; Cai, L. Peptide sequence tag-based blind identification of post-translational modifications with point process model. Bioinformatics. 2006, 22, e307-e313.

150. Liu, Y.; Li, X.; Li, K.; Liu, H.; Lin, C. Multiple bHLH proteins form heterodimers to mediate CRY2-dependent regulation of flowering-time in Arabidopsis. PLoS Genet. 2013, 9, e1003861.

151. Liu, Z.; Miao, L.; Huo, R.; Song, X.; Johnson, C.; Kong, L.; Sundaresan, V.; Yu, X. ARF2-ARF4 and ARF5 are essential for female and male gametophyte development in Arabidopsis. Plant Cell Physiol. 2018, 59, 179-189.

152. Lobstein, E.; Guyon, A.; Férault, M.; Twell, D.; Pelletier, G.; Bonhomme, S. The putative Arabidopsis homolog of yeast Vps52p is required for pollen tube elongation, localizes to Golgi, and might be involved in vesicle trafficking. Plant Physiol. 2004, 135, 1480-1490.

153. Lolle, S. J.; Pruitt, R. E. Epidermal cell interactions: a case for local talk. Trends Plant Sci. 1999, 4, 14-20.

154. Lora, J.; Herrero, M.; Hormaza, J. I. The coexistence of bicellular and tricellular pollen in Annona cherimola (Annonaceae): Implications for pollen evolution. Am. J. Bot. $2009,96,802-808$.

155. Lora, J.; Hormaza, J. I.; Herrero, M. The diversity of the pollen tube pathway in plants: Toward an increasing control by the sporophyte. Front. Plant Sci. 2016, 7, 107. 
156. Lord, E. M.; Russell, S. D. The mechanisms of pollen and fertilization in plants. Annu. Rev. Cell Dev. Biol. 2002, 18, 81-105.

157. Luo, X.; Chen, Z.; Gao, J.; Gong, Z. Abscisic acid inhibits root growth in Arabidopsis through ethylene biosynthesis. Plant J. 2014, 79, 44-55.

158. Lysak, M. A.; Koch, M. A.; Pecinka, A.; Schubert, I. Chromosome triplication found across the tribe Brassiceae. Genome Res. 2005, 15, 516-525.

159. Martinez-Fernández, I.; Sanchis, S.; Marini, N.; Balanzá, V.; Ballester, P.; Navarrete-Gómez, M.; Oliveira, A. C.; Colombo, L.; Ferrándiz, C. The effect of NGATHA altered activity on auxin signaling pathways within the Arabidopsis gynoecium. Front. Plant Sci. 2014, 5, 201.

160. Magdelin, S.; Enany, S.; Yoshida, Y.; Xu, B.; Zhang, Y.; Zureena, Z.; Lokamani, I.; Yaoita, E.; Yamamoto, T. Basics and recent advances of two dimensionalpolyacrylamide gel electrophoresis. Clin. Proteomics. 2014, 11, 16.

161. Mauseth, J. D. Botany: An introduction to plant biology, $2^{\text {nd }}$ ed.; Jones and Bartlett Publishers: Sudbury, MA, USA, 1998; pp 228- 249.

162. McHugh, L.; Arthur, J. W. Computational methods for protein identification from mass spectrometry data. PLoS Comput. Biol. 2008, 4, e12.

163. McInnis, S. M.; Emery, D. C.; Porter, R.; Desikan, R.; Hancock, J. T.; Hiscock, S. J. The role of stigma peroxidases in flowering plants: insights from further characterization of a stigma-specific peroxidase (SSP) from Senecio squalidus (Asteraceae). J. Exp. Bot. 2006, 57, 1835-1846. 
164. Meng, X.; Chen, X.; Mang, H.; Liu, C.; Yu, X.; Gao, X.; Torii, K. U.; He, P.; Shan, L. Differential function of Arabidopsis SERK family receptor-like kinases in stomatal patterning. Curr. Biol. 2015, 25, 2361-2372.

165. Meyer, S.; Reverchon, S.; Nasser, W.; Muskhelishvili, G. Chromosomal organization of transcription: in a nutshell. Curr. Genet. 2018, 64, 555-565.

166. Mi H.; Huang, X.; Muruganujan, A.; Tang, H.; Mills, C.; Kang, D.; Thomas, P. D. PANTHER version 11: expanded annotation data from Gene Ontology and Reactome pathways, and data analysis tool enhancements. Nucleic Acids Res. 2017, 45, D183D189.

167. Min, M. K.; Jang, M.; Lee, M.; Lee, J.; Song, K.; lee, Y.; Choi, K. Y.; Robinson, D. G.; Hwang, I. Recruitment of Arfl-GDP to golgi by Glo3p-type ArfGAPs is crucial for golgi maintenance and plant growth. Plant Physiol. 2013, 161, 676-691.

168. Mironov, V.; De Veylder, L.; Van Montagu, M.; Inzé, D. Cyclin-dependent kinases and cell division in plants - The Nexus. Plant Cell, 1999, 11, 509-521.

169. Molina, I.; Ohlrogge, J. B.; Pollard, M. Deposition and localization of lipid polyester in developing seeds of Brassica napus and Arabidopsis thaliana. Plant J. 2008, $53,437-449$.

170. Mollet, J.; Leroux, C.; Dardelle, F.; Lehner, A. Cell wall composition, biosynthesis and remodeling during pollen tube growth. Plants. 2013, 2, 107-147.

171. Moubayidin, L.; Østergaard, L. Dynamic control of auxin distribution imposes a bilateral-to-radial symmetry switch during gynoecium development. Curr. Biol. 2014, 24, 2743-2748. 
172. Mussury, R. M.; Fernandes, W. D. Studies of the floral biology and reproductive system of Brassica napus L. (Cruciferae). Braz. Arch. Biol. Technol. 2000, 43, 111-117.

173. Myers, C.; Romanowsky, S. M.; Barron, Y. D.; Garg, S.; Azuse, C. L.; Curran, A.; Davis, R. M.; Hatton, J.; Harmon, A. C.; Harper, J. F. Calcium-dependent protein kinases regulate polarized tip growth in pollen tubes. Plant J. 2009, 59, 528-539.

174. Nagano, T.; Lubling, Y.; Várnai, C.; Dudley, C.; Leung, W.; Baran, Y.; Mendelson-Cohen, N.; Wingett, S.; Fraser, P.; Tanay, A. Cell-cycle dynamics of chromosomal organization at single cell resolution. Nature. 2017, 547, 61-67.

175. Nakamura, M.; Katsumata, H.; Abe, M.; Yabe, N.; Komeda, Y.; Yamamoto, K. T.; Takahashi, T. Characterization of the class IV homeodomain-leucine zipper gene family in Arabidopsis. Plant Physiol. 2006, 141, 1363-1375.

176. Nawkar, G. M.; Kang, C. H.; Maibam, P.; Park, J. H.; Jung, Y. J.; Chae, H. B.; Chi, Y. H.; Jung, I. J.; Kim, W. Y.; Yun, D.; Lee, S. Y. HY5, a positive regulator of light signaling, negatively controls the unfolded protein response in Arabidopsis. PNAS. 2017, DOI: doi.org/10.1073/pnas.1609844114

177. Nasrallah, J. B. Recognition and rejection of self in plant reproduction. Science. 2002, 296, 305-308.

178. Nazemof, N.; Couroux, P.; Rampitsch, C.; Xing, T.; Robert, L. S. Proteomic profiling reveals insights into Triticeae stigma development and function. J. Exp. Bot. 2014, 65, 6069-6080.

179. Nazemof, N.; Couroux, P.; Xing, T.; Robert, L. S. Proteomic analysis of the mature Brassica stigma reveals proteins with diverse roles in vegetative and reproductive development. Plant Sci. 2016, 250, 51-58. 
180. Nesvizhskii, A. I.; Keller, A.; Kolker, E.; Aebersold, R. A statistical model for identifying proteins by tandem mass spectrometry. Anal. Chem. 2003, 75, 4646-4658.

181. Nissen, K. S.; Willats, W. G. T.; Malinovsky, F. G. Understanding CrRLK1L function: Cell walls and growth control. Trends Plant Sci. 2016, 6, 516-527.

182. Noir, S.; Bräutigam, A.; Colby, T.; Schmidt, J.; Panstruga, R. A reference map of the Arabidopsis thaliana mature pollen proteome. Biochem. Biophys. Res. Commun. $2005,337,1257-1266$.

183. Nowack, M. K.; Harashima, H.; Dissmeyer, N.; Zhao, X.; Bouyer, D.; Weimer, A. K.; Winter, F. D.; Yang, F.; Schnittger, A. Genetic framework of cyclin-dependent kinase function in Arabidopsis. Dev. Cell. 2012, 22, 1030-1040.

184. Oberg, A.; Mahoney, D. W.; Eckel-Passow, J. E.; Malone, C. J.; Wolfinger, R. D.; Hill, E. G.; Cooper, L. T.; Onuma, O. K.; Spiro, C.; Therneau, T. M.; Bergen III, H. R. Statistical analysis of relative labeled mass spectrometry data from complex samples using ANOVA. J. Proteome Res. 2008, 7, 225-233.

185. Obermeyer, G.; Fragner, L.; Lang, V.; Weckwerth, W. Dynamic adaptation of metabolic pathways during germination and growth of lily pollen tubes after inhibition of the electron transport chain. Plant Physiol. 2013, 162, 1822-1833.

186. Oettler, G. The fortune of a botanical curiosity - Triticale: past; present and future. J. Agric. Sci. 2005, 143, 329-346.

187. Paez Valenxia, J.; Goodman, K.; Otegui, M. S. Endocytosis and endosomal trafficking in plants. Annu. Rev. Plant Biol. 2016, 67, 309-335. 
188. Parke, Y.; Runkle, E. S. Far-red radiation promotes growth of seedlings by increasing leaf expansion and whole-plant net assimilation. Environ. Exp. Bot. 2017, 136, 41-49.

189. Paxson- Sowders, D. M.; Dodrill, C. H.; Owen, H. A.; Makaroff, C. A. DEX1, a novel plant protein, is required for exine pattern formation during pollen development in Arabidopsis. Plant Physiol. 2001, 127, 1739-1749.

190. Pelaz, S.; Ditta, G. S.; Baumann, E.; Wisman, E.; Yanofsky, M. F. B and C floral organ identity functions require SEPALLATA MADS-box genes. Nature. 2000, 405, 200203.

191. Peng, J.; Ilarslan, H.; Wurtele, E. S.; Bassham, D. C. 5AtRabD2b and AtRabD2c have overlapping functions in pollen development and pollen tube growth. BMC Plant Biol. 2011, 11, 25.

192. Pérez-Pérez, J. M.; Ponce, M. R.; Micol, J. L. The UCU1 Arabidopsis gene encodes a SHAGGY/GSK3-like kinase required for cell expansion along the proximodistal axis. Dev. Biol. 2002, 242, 161-173.

193. Potocký, M.; Pejchar, P.; Gutkowska, M.; Jiménez-Quesada, M. J.; Potocká, A.; Alché, J.; Kost, B.; Žárský, V. NADPH oxidase activity in pollen tubes is affected by calcium ions, signaling phospholipids and Rac/Rop GTPases. J. Plant Physiol. 2012, 169, 1654-1663.

194. Qin, L.; Nie, X.; Hu, R.; Li, G.; Xu, W.; Li, X. Phosphorylation of serine residue modulates cotton Di19-1 and Di19-2 activities for responding to high salinity stress and abscisic acid signaling. Sci. Rep. 2016, DOI: 10.1038/srep20371. 
195. Qin, Y.; Yang, Z. Rapid tip growth: Insights from pollen tubes. Semin. Cell. Dev. Biol. 2011, 22, 816-824.

196. Rademacher, E. H.; Möller, B.; Lokerse, A. S.; Llavata-Peris, C. I.; van den Berg, W.; Weijers, D. A cellular expression map of the Arabidopsis AUXIN RESPONSE FACTOR gene family. Plant J. 2011, 68, 597-606.

197. Rakow, G. Species origin and economic importance of Brassica. In Biotechnology in Agriculture and Forestry; Pua, E. C.; Douglas, C. J., Ed.; Springer: Berlin, Germany, 2004; pp 3-5.

198. Rehman, S.; Yun, S. J. Developmental regulation of K accumulation in pollen, anthers, and papillae: are anther dehiscence, papillae hydration, and pollen swelling leading to pollination and fertilization in barley (Hordeum vulgare L.) regulated by changes in K concentration? J. Exp. Bot. 2006, 57, 1315-1321.

199. Rejón, J. D.; Delalande, F.; Schaeffer-Reiss, C.; Alché, J.; Rodríguez-García, M. I.; Dorsselaer, A. V.; Castro, A. J. The pollen coat proteome: At the cutting edge of plant reproduction. Proteomes. 2016, DOI: 10.3390/proteomes4010005.

200. Resing, K. A.; Ahn, N. G. Proteomics strategies for protein identification. FEBS Lett. 2005, 579, 885-889.

201. Reyes-Olalde, J. I.; Zuñiga-Mayo, V. M.; Chávez Montes, R. A.; MarschMartínez, N.; de Folter, S. Inside the gynoecium: at the carpel margin. Trends in Plant Sci. 2013, 18, 644-655.

202. Ride, J. P.; Davies, E. M.; Franklin, F. C. H.; Marshall, D. F. Analysis of Arabidopsis genome sequence reveals a large new gene family in plants. Plant Mol. Biol. 1999, 39, 927-932. 
203. Robert, L. S.; Allard, S.; Franklin, T. M.; Trick, M. Sequence and expression of endogenous $S$-locus glycoprotein genes in self-compatible Brassica napus. Mol. Gen. Genet. 1994, 242, 209-216.

204. Rowland, O.; Zheng, H.; Hepworth, S. R.; Lam, P.; Jetter, R.; Kunst, L. CER4 encodes an alcohol-forming fatty acyl-coenzyme A reductase involved in cuticular wax production in Arabidopsis. Plant Physiol. 2006, 142, 866-877.

205. Ruprecht, C.; Mendrinna, A.; Tohge, T.; Sampathkumar, A.; Klie, S.; Fernie, A. R.; Nikoloski, Z.; Persson, S.; Mutwil, M. FamNet: A framework to identify multiplied modules driving pathway expansion in plants. Plant Physiol. 2016, 170, 1878-1894.

206. Saedler, H.; Becker, A.; Winter, K.; Kirchner, C.; Theißen, G. MADS-box genes are involved in floral development and evolution. Acta Biochim. Pol. 2001, 48, 351-358. 207. Safavian, D.; Zayed, Y.; Indriolo, E.; Chapman, L.; Ahmed, A.; Goring, D. R. RNA silencing of exocyst genes in the stigma impairs the acceptance of compatible pollen in Arabidopsis. Plant Physiol. 2015, 169, 2526-2538.

208. Samuel, M. A.; Chong, Y. T.; Hassen, K. E.; Aldea-Brydges, M. G.; Stone, S. L.; Goring, D. R. Cellular pathways regulating responses to compatible and selfincompatible pollen in Brassica and Arabidopsis stigmas intersect at EXO70A1, a putative component of the exocyst complex. Plant Cell. 2009, 21, 2655-2671.

209. Samuel, M. A.; Tang, W.; Jamshed, M.; Northey, J.; Patel, D.; Smith, D.; Siu, K. W. M.; Muench, D. G.; Wang, Z. Y.; Goring, D. R. Proteomic analysis of Brassica stigmatic proteins following the self-incompatibility reaction reveals a role for microtubule dynamics during pollen responses. Mol. Cell. Proteomics. 2011, 10, 1-13. 
210. Sang, Y. L.; Xu, M.; Ma, F. F.; Chen, H.; Xu, X. H.; Gao, X. Q.; Zhang, X. S. Comparative proteomic analysis reveals similar and distinct features of proteins in dry and wet stigmas. Proteomics 2012, 12, 1983-1998.

211. Sansebastiano, G. D.; Prio, G. The SNARE proteins (in plants) beyond the Nobel prize. J. Plant Biochem. Physiol. 2014, DOI: 10.4172/2329-9029.1000e122.

212. Schoenaers, S.; Balcerowicz, D.; Vissenberg, K. Molecular mechanisms regulating root hair tip growth: A comparison with pollen tubes. In Pollen Tip Growth; Obermeyer, G.; Feijó, J. Ed.; Springer: Berlin, Germany, 2017; pp 167-243.

213. Serrazina, S.; Vas Dias, F.; Malhó, R. Characterization of FAB1 phosphatidylinositol kinase in Arabidopsis pollen tube growth and fertilization. New Phytol. 2014, 203, 784-793.

214. Schiøtt, M.; Romanowsky, S. M.; Bækgaard, L.; Jakobsen, M. K.; Palmgren, M. G.; Harper, J. F. A plant plasma membrane $\mathrm{Ca}^{2+}$ pump is required for normal pollen tube growth and fertilization. PNAS. 2004, 101, 9502-9507.

215. Schnurr, J.; Shockey, J.; Browse, J. The acyl-CoA synthetase encoded by LACS2 is essential for normal cuticle development in Arabidopsis. Plant Cell. 2004, 16, 629-642.

216. Schruff, M. C.; Spielman, M.; Tiwari, S.; Adams, S.; Fenby, N.; Scott, R. J. the AUXIN RESPONSE FACTOR 2 gene of Arabidopsis links auxin signaling, cell division, and the size of seeds and other organs. Development. 2006, 133, 251-261.

217. Schulz, P.; Herde, M.; Romeis, T. Calcium-dependent protein kinases: Hubs in plant stress signaling and development. Plant Physiol. 2013, 163, 523-530.

218. Selinski, J.; Scheibe, R. Pollen tube growth: where does the energy come from? Plant Signal Behav. 2014, DOI: 10.4161/15592324.2014.977200. 
219. Senko, M. W.; Remes, P. M.; Canterbury, J. D.; Mathur, R.; Song, Q.; Eliuk, S. M.; Mullen, C.; Earley, L.; Hardman, M.; Blethrow, J. D.; Bui, H.; Specht, A.; Lange, O.; Denisov, E.; Markarov, A.; Horning, S.; Zabrouskov, V. Novel parallelized Quadrupole/Linear Ion Trap/Orbitrap Tribrid Mass Spectrometer improving proteome coverage and peptide identification rates. Anal. Chem. 2013, 85, 11710-11714.

220. Shaikhali, J.; de Dios Barajas-Lopéz, J.; Ötvös, K.; Kremnev, D.; Garcia, A. S.; Srivastava, V.; Wingsle, G.; Bako, L.; Strand, Å. The CRYPTOCHROME1-dependent response to excess light is mediated through the transcriptional activators ZINC FINGER PROTEIN EXPRESSED IN INFLORESCENCE MERISTEM LIKE1 and ZML2 in Arabidopsis. Plant Cell. 2012, 24, 3009-3025.

221. Shani, Z.; Dekel, M.; Roiz, L.; Horowitz, M.; Kolosovski, N.; Lapidot, S.; Alkan, S.; Koltai, H.; Tsabary, G.; Goren, R.; Shoseyov, O. Expression of endo-1,4-betaglucanase (cel1) in Arabidopsis thaliana is associated with plant growth, xylem development and cell wall thickening. Plant Cell Rep. 2006, 25, 1067-1074.

222. Sheng, X.; Hu, Z.; Lü, H.; Wang, X.; Baluška, F.; Šamaj, J.; Lin, J. Roles of the ubiquitin/proteasome pathway in pollen tube growth with emphasis on MG132-induced alterations in ultrastructure, cytoskeleton, and cell wall components. Plant Physiol, 2006, $141,1578-1590$.

223. Sheoran, I. S.; Pedersen, E. J.; Ross, A. R. S.; Sawhney, V. K. Dynamics of protein expression during pollen germination in canola (Brassica napus). Planta. 2009, 230, 779- 793. 
224. Sheoran, I. S.; Sproule, K. A.; Olson, D. J. H.; Ross, A. R. S.; Sawhney, V. K. Proteome profile and functional classification of proteins in Arabidopsis thaliana (Landsberg erecta) mature pollen. Sex. Plant Reprod. 2006, 19, 185-196.

225. Shinozuka, H.; Cogan, N. O. I.; Smith, K. F.; Spangenberg, G. C.; Forster, J. W. Fine-scale comparative genetic and physical mapping supports map-based cloning strategies for the self-incompatibility loci of perennial ryegrass (Lolium perenne L.). Plant Mol. Biol. 2010, 72, 343-355.

226. Showalter, A. M.; Basu, D. Extensin and arabinogalactan protein biosynthesis: glycosyltransferases, research challenges and biosensors. Front. Plant Sci. 2016, DOI: doi.org/10.3389/fpls.2016.00814

227. Siddiqui, H.; Khan, S.; Rhodes, B. M.; Devlin, P. F. FHY3 and FAR1 act downstream of light stable phytochromes. Front. Plant Sci. 2016, DOI:

$10.3389 /$ fpls.2016.00175

228. Silva, P. A.; Ul-Rehman, R.; Rato, C.; Sansebastiano, G. P. D.; Malhó, R. Asymmetric localization of Arabidopsis SYP124 syntaxin at the pollen tube apical and sub-apical zones is involved in tip growth. BMC Plant Biol. 2010, 10, 179.

229. Sklodowski, K.; Riedelsberger, J.; Raddatz, N.; Riadi, G.; Caballero, J.; Chérel, I.; Schulze, W.; Graf, A.; Dreyer, I. The receptor-like pseudokinase MRH1 interacts with the voltage gated potassium channel AKT2. Sci. Rep. 2017, DOI: 10.1038/srep44611. 230. Slane, D.; Reichardt, I.; Kasmi, F. E.; Bayer, M.; Jürgens, G. Evolutionarily diverse SYP1 Qa-SNAREs jointly sustain pollen tube growth in Arabidopsis. Plant J. 2017, 92, 375-385. 
231. Smirnova, A. V.; Matveyeva, N. P.; Polesskaya, O. G.; Yermakov, I. P. Generation of reactive oxygen species during pollen grain germination. Russ. J. Dev. Biol. 2009, 40, 345-353.

232. Smith, D. K.; Jones, D. M.; Lau, J. B. R.; Cruz, E. R.; Brown, E.; Harper, J. F.; Wallace, I. S. A putative protein $O$-fucosyltransferase facilitates pollen tube penetration through the stigma-style interface. Plant Physiol. 2018, 176, 2804-2818.

233. Soltis, D. E.; Misra, B. B.; Shan, S.; Chen, S.; Soltis, P. S. Polyploidy and the proteome. Biochim. Biophys. Acta. 2016, 1864, 896-907.

234. Song, L.; Zou, J.; Zhang, W.; Wu, W.; Wang, Y. Ion transporters involved in pollen germination and pollen tube tip-growth. Plant Signaling Behav. 2009, 4, 1193 1195.

235. Soto, G.; Alleva, K.; Mazzella, M. A.; Amodeo, G.; Muschietti, J. P. AtTIP1;3 and AtTIP 5; 1, the only highly expressed Arabidopsis pollen-specific aquaporins, transport water and urea. FEBS Lett. 2008, 582, 4077-4082.

236. Speranza, A.; Crinelli, R.; Scoccianti, V.; Geitmann, A. Reactive oxygen species are involved in pollen tube initiation in kiwifruit. Plant Biol. (Stuttg). 2012, 14, 64-76. 237. Steinhorst, L.; Kudla, J. Calcium - a central regulator of pollen germination and tube growth. Biochim. Biophys. Acta. 2013, 1833, 1573-1581.

238. Steinhorst, L.; Mähs, A.; Ischebeck, T.; Zhang, C.; Zhang, X.; Arendt, S.; Schültke, S.; Heilmann, I.; Kudla, J. Vacuolar CBL-CIPK12 $\mathrm{Ca}^{2+}$ - sensor-kinase complexes are required for polarized pollen tube growth. Curr. Biol. 2015, 25, 14751482. 
239. Suen, D. F.; Huang, A. H. C. Maize pollen coat xylanase facilitates pollen tube penetration into silk during sexual reproduction. J. Biol. Chem. 2007, 282, 625-636.

240. Sze, H.; Padmanaban, S.; Cellier, F.; Honys, D.; Cheng, N. H.; Bock, K. W.; Conéjéro, G.; Li, X.; Twell, D.; Ward, J. M.; Hirschi, K. D. Expression patterns of a novel $\mathrm{AtCHX}$ gene family highlight potential roles in osmotic adjustment and $\mathrm{K}^{+}$ homeostasis in pollen development. Plant Physiol. 2004, 136, 2532-2547.

241. Szewińska, J.; Zdunek-Zastocka, E.; Pojmaj, M.; Bielawski, W. Molecular cloning and expression analysis of triticale phytocystatins during development and germination of seeds. Plant Mol. Biol. Rep. 2012, 30, 867-877.

242. Szklarczyk, D.; Morris, J. H.; Cook, H.; Kuhn, M.; Wyder, S.; Simonovic, M.; Santos, A.; Doncheva, N. T.; Roth, A.; Bork, P.; Jensen, L. J.; von Mering, C. The STRING database in 2017: quality-controlled protein-protein association networks, made broadly accessible. Nucleic Acids Res. 2017, 45, D362-D368.

243. Szumlanski, A. L.; Nielsen, E. The Rab GTPase RabA4d regulates pollen tube tip growth in Arabidopsis thaliana. Plant Cell. 2009, 21, 526-544.

244. Takahashi, F.; Mizoguchi, T.; Yoshida, R.; Ichimura, K.; Shinozaki, K. Calmodulin-dependent activation of MAP kinase for ROS homeostasis in Arabidopsis. Mol. Cell. 2011, 6, 649-660.

245. Takahashi, K.; Shimada, T.; Kondo, M.; Tamai, A.; Mori, M.; Nishimura, M.; Hara-Nishimura, I. Ectopic expression of an esterase, which is a candidate for the unidentified plant cutinase, causes cuticular defects in Arabidopsis thaliana. Plant Cell Physiol. 2010, 51, 123-131. 
246. Takatsuka, H.; Ohno, R.; Umeda, M. The Arabidopsis cyclin-dependent kinaseactivating kinase CDKF; 1 is a major regulator of cell proliferation and cell expansion but is dispensable for CDKA activation. Plant J. 2009, 59, 457-487.

247. Takatsuka, H.; Umeda-Hara, C.; Umeda, M. Cyclin-dependent kinase-activating kinases CDKD; 1 and CDKD;3 are essential for preserving mitotic activity in Arabidopsis thaliana. Plant J. 2015, 82, 1004-1017.

248. Tan, X.; Cao, K.; Liu, F.; Li, Y.; Li, P.; Gao, C.; Ding, Y.; Lan, Z.; Shi, Z.; Rui, Q.; Feng, Y.; Liu, Y.; Zhao, Y.; Wu, C.; Zhang, Q.; Li, Y.; Jiang, L.; Bao, Y. Arabidopsis COG complex subunits COG3 and COG8 modulate golgi morphology, vesicle trafficking homeostasis and are essential for pollen tube growth. PLoS Genet. 2016, 12, e1006140.

249. Thorogood, D.; Yates, S.; Manzanares, C.; Skot, L.; Hegarty, M.; Blackmore, T.; Barth, S.; Studer, B. A novel multivariate approach to phenotyping and association mapping of multi-locus gametophytic self-incompatibility reveals S; Z; and other loci in a perennial ryegrass (Poaceae) population. Front. Plant Sci. 2017, 8, 1331.

250. Trigueros, M.; Navarrete-Gómez, M.; Sato, S.; Christensen, S. K.; Pelaz, S.;

Weigel, D.; Yanofsky, M. F.; Ferrándiz, C. The NGATHA genes direct style development in the Arabidopsis gynoecium. Plant Cell. 2009, 21, 1394-1409.

251. Tran, F.; Penniket, C.; Patel, R. V.; Provart, N. J.; Laroche, A.; Rowland, O.; Robert, L. S. Developmental transcriptional profiling reveals key insights into Triticeae reproductive development. Plant J. 2013, 74, 971-988.

252. Treu, R.; Emberlin, J. Pollen dispersal in the crops Maize (Zea mays), Oil seed rape (Brassica napus ssp. oleifera), Potatoes (Solanum tuberosum), Sugar beet (Beta vulgaris ssp. vulgaris) and Wheat (Triticum aestivum). A report for the Soil Association 
from the National Pollen Research Unit; University College: Worcester, UK, 2000; http://ibrarian.net/navon/paper/Pollen_dispersal_in_the_crops_Maize_Zea _mays__o.pdf?paperid=2943414.

253. Tsai, A. Y.; Gazzarrini, S. AKIN10 and FUSCA3 interact to control lateral organ development and phase transitions in Arabidopsis. Plant J. 2012, 69, 809-821.

254. Varaud, E.; Brioudes, F.; Szécsi, J.; Leroux, J.; Brown, S.; Perrot-Rechenmann, C.; Bendahmane, M. AUXIN RESPONSE FACTOR8 regulates Arabidopsis petal growth by interacting with the bHLH transcription factor BIGPETALp. Plant Cell. 2011, 23, 973-983.

255. Velasquez, S. M.; Ricardi, M. M.; Dorosz, J. G.; Fernandez, P. V.; Nadra, A. D.; Pol-Fachin, L.; Egelund, J.; Gille, S.; Harholt, J.; Ciancia, M.; Verli, H.; Pauly, M.; Bacic, A.; Olsen, C. E.; Ulvskov, P.; Petersen, B. L.; Somerville, C.; Iusem, N. D.; Estevez, J. M. O-glycosylated cell wall proteins are essential in root hair growth. Science. 2011, 332, 1401-1403.

256. Velasquez, S. M.; Ricardi, M. M.; Poulsen, C. P.; Oikawa, A. Dilokpimol, A.; Halim, A.; Mangano, S.; Juarez, S. P. D.; Marzol, E.; Salter, J. D. S.; Dorosz, J. G.; Borassi, C.; Möller, S. R.; Buono, R.; Ohsawa, Y.; Matsuoka, K.; Otegui, M. S.; Scheller, H. V.; Geshi, N.; Petersen, B. L.; Iusem, N. D.; Estevez, J. M. Complex regulation of prolyl-4-hydroxylases impacts root hair expansion. Mol. Plant. 8, 734-746.

257. Verger, S.; Chabout, S.; Gineau, E.; Mouille, G. Cell adhesion in plants is under the control of putative O-fucosyltransferases. Development. 2016, 143, 2536-2540.

258. Vines, S. H. A student's text-book of Botany: Volume 1; Swan Sonnenschein \& Co: London, UK, 1894; pp 67-88. 
259. Vogler, F.; Schmalzl, C.; Englhart, M.; Bircheneder, M.; Sprunck, S.

Brassinosteroids promote Arabidopsis pollen germination and growth. Plant Reprod. 2014, 27, 153-167.

260. Vollmann, J.; Rajca, I. Oil Crops; Springer: New York; NY, 2009; pp 129.

261. Wang, H.; Liu, Y.; Bruffett, K.; Lee, J.; Hause, G.; Walker, J. C.; Zhang, S.

Haplo-insufficiency of $M P K 3$ in $M P K 6$ mutant background uncovers a novel function of these two MAPKs in Arabidopsis ovule development. Plant Cell. 2008, 20, 602-613.

262. Wang, H.; Tse, Y. C.; Law, A. H. Y.; Sun, S. S. M.; Sun, Y.; Xu, Z.; Hillmer, S.;

Robinson, D. G.; Jiang, L. Vacuolar sorting receptors (VSRs) and secretory carrier membrane proteins (SCAMPs) are essential for pollen tube growth. Plant J. 2010, 61, 826-838.

263. Wang, L.; Wang, W.; Wang, Y.; Liu, Y.; Wang, J.; Zhang, X.; Ye, D.; Chen, L. Arabidopsis galacturonosyltransferase (GAUT) 13 and GAUT14 have redundant functions in pollen tube growth. Mol. Plant. 2013, 6, 1131-1148.

264. Wang, L.; Yeh, C.; Sayler, R. J.; Lee, Y.; Lu, C.; Wu, S. Arabidopsis HIT1, a putative homolog of yeast tethering protein Vps53p, is required for pollen tube elongation. Bot. Stud. 2008, 49, 25-32.

265. Wang, T.; Liang, L.; Xue, Y.; Jia, P.; Chen, W.; Zhang, M.; Wang, Y.; Li, H.; Yang, W. A receptor heteromer mediates the male perception of female attractants in plants. Nature. 2016, 531, 241-244.

266. Wang, W.; Chen, X. HUA ENHANCER3 reveals a role for a cyclin-dependent protein kinase in the specification of floral organ identity in Arabidopsis. Development. 2004, 131, 3147-3156. 
267. Wang, W.; Tang, W.; Ma, T.; Niu, D.; Jin, J. B.; Wang, H.; Lin, R. A pair of light signaling factors FHY3 and FAR1 regulates plant immunity by modulating chlorophyll biosynthesis. J. Integr. Plant Biol. 2016, 58, 91-103.

268. Wang, Y.; Henriksson, E.; Söderman, E.; Henriksson, K. N.; Sunberg, E.; Engström, P. The Arabidopsis homeobox gene, $A T H B 16$, regulates leaf development and the sensitivity to photoperiod in Arabidopsis. Dev. Biol. 2003, 264, 228-239.

269. Wagner, D.; Meyerowitz, E. M. SPLAYED, a novel SWI/SNF ATPase homolog controls reproductive development in Arabidopsis. Curr. Biol. 2002, 12, 85-94.

270. Wengier, D. L.; Lampard, G. R.; Bergmann, D. C. Dissection of MAPK signaling specificity through protein engineering in a developmental context. BMC Plant Biol. 2018, DOI: doi.org/10.1186/s12870-018-1274-9.

271. Wilhelmi, L. K.; Preuss, D. Self-sterility in Arabidopsis due to defective pollen tube guidance. Science, 1996, 274, 1535-1537.

272. Willson, M. F. Sexual selection and dicliny in angiosperms. Am. Nat. 1982, 119, $579-583$.

273. Wissing, J.; Jänsch, L.; Nimtz, M.; Dieterich, G.; Hornberger, R.; Kéri, G.; Wehland, J.; Daub, H. Proteomics analysis of protein kinases by target class-selective prefractionation and tandem mass spectrometry. Mol. Cell Proteomics. 2007, 6, 537-547.

274. Wu, M.; Tian, Q.; Reed, J. W. Arabidopsis microRNA167 controls patterns of $A R F 6$ and $A R F 8$ expression, and regulates both female and male reproduction. Development. 2006, 133, 4211-4218. 
275. Wu, Y.; Zhao, S.; Tian, H.; He, Y.; Xiong, W.; Guo, L; Wu, Y. CPK3-

phosphorylated RhoGDI1 is essential in the development of Arabidopsis seedlings and leaf epidermal cells. J. Exp. Bot. 2013, 64, 3327-3338.

276. Xiao, F.; Goodwin, S. M.; Xiao, Y.; Sun, Z.; Baker, D.; Tang, X.; Jenks, M. A.; Zhou J. M. Arabidopsis CYP86A2 represses Pseudomonas syringae type III genes and is required for cuticle development. EMBO J. 2004, 23, 2903-2913.

277. Xu, T.; Dai, N.; Chen, J.; Nagawa, S.; Cao, M.; Li, H.; Zhou, Z.; Chen, X.; De Rycke, R.; Rakusová, H.; Wang, W.; Jones, A. M.; Friml, J.; Patterson, S. E.; Bleecker, A. B.; Yang, Z. Cell surface ABP1-TMK auxin-sensing complex activates ROP GTPase signaling. Science. 2014, 343, 1025-1028.

278. Xu, P.; Jiao, Z.; Wang, Z.; Ai, J.; Yang, Y.; Zhang, Q.; Qin, H.; Liu, Y.; Zhao, Y.; Fan, S. Proteomic analysis of sex conversion induced by CPPU in male grapevine of Vitis amurensis. Vitis. 2013, 52, 177-184.

279. Yang, C.; Li, H.; Zhang, J.; Wang, T.; Ye, Z. Fine-mapping of the woolly gene controlling multicellular trichome formation and embryonic development in tomato. Theor. Appl. Genet. 2011, 123, 625-633.

280. Yang, F.; Shen, Y.; Camp II, D. G.; Smith, R. D. High pH reversed-phase chromatography with fraction concatenation as an alternative to strong-cation exchange chromatography for two-dimensional proteomic analysis. Expert Rev. Proteomics. 2012, 9, 129-134.

281. Yang, J.; Wu, J.; Romanovicz, D.; Clark, G.; Roux, S. J. Co-regulation of exine wall patterning, pollen fertility and anther dehiscence by Arabidopsis apyrases 6 and 7 . Plant Physiol. Biochem. 2013, 69, 62-73. 
282. Yang, Y.; Dong, C.; Yu, J.; Shi, L.; Tong, C.; Li, Z.; Huang, J.; Liu, S. Cysteine Protease 51 (CP51), an anther-specific cysteine protease gene, is essential for pollen exine formation in Arabidopsis. Plant Cell, Tissue Organ Cult. 2014, 119, 383-397.

283. Yates, J. R.; Ruse, C. I.; Nakorchevsky, A. Proteomics by mass spectrometry: approaches, advances, and applications. Annu. Rev. Biomed. Eng. 2009, 11, 49-79.

284. Ye, Q.; Zhu, W.; Li, L.; Zhang, S.; Yin, Y.; Ma, H.; Wang, X. Brassinosteroids control male fertility by regulating the expression of key genes involved in Arabidopsis anther and pollen development. PNAS. 2010, 107, 6100-6105.

285. Yeung, K. C.; Larter, E. N. Pollen production and disseminating properties of triticale relative to wheat. Can. J. Plant Sci. 1972, 52, 569-574.

286. Yue, X.; Gao, X.; Wang, F.; Dong, Y.; Li, X.; Zhang, X. S. Transcriptional evidence for inferred pattern of pollen tube-stigma metabolic coupling during pollination. PLoS One. 2014, 9, e107046.

287. Zaidi, M. A.; O’Leary, S.; Wu, S.; Gleddie, S.; Eudes, F.; Laroche, A.; Robert, L. S. A molecular and proteomic investigation of proteins rapidly released from triticale pollen upon hydration. Plant Mol. Biol. 2012, 79, 101-121.

288. Zhang, Y.; Fonslow, B. R.; Shan, B.; Baek, M. C.; Yates, J. R. Protein analysis by shotgun/bottom-up proteomics. Chem. Rev. 2013, 113, 2343-2394.

289. Zhang, Y.; He, J.; McCormick, S. Two Arabidopsis AGC kinases are critical for the polarized growth of pollen tubes. Plant J. 2009, 58, 474-484.

290. Zhao, L.; Shen, Li.; Zhang, W.; Zhang, W.; Wang, Y.; Wu, W. Ca ${ }^{2+}$ - Dependent Protein Kinase 11 and 24 modulate the activity of the inward rectifying $\mathrm{K}^{+}$channels in Arabidopsis pollen tubes. Plant Cell. 2013, 25, 649-661. 
291. Zheng, H.; Rowland, O.; Kunst, L. Disruptions of the Arabidopsis enoyl-CoA reductase gene reveal an essential role for very-long-chain fatty acid synthesis in cell expansion during plant morphogenesis. Plant Cell. 2005, 17, 1467-1481.

292. Zhu, S.; Yu, X.; Wang, X.; Zhao, R.; Li, Y.; Fan. R.; Shang, Y.; Du, S.; Wang, X.; Wu, F.; Xu, Y.; Zhang, X.; Zhang, D. Two calcium-dependent protein kinases, CPK4 and CPK11, regulate abscisic acid signal transduction in Arabidopsis. Plant Cell. 2007, 19, 3019-3036.

293. Zhu, Y.; Zhao, P.; Wu, X.; Wang, W.; Scali, M.; Cresti, M. Proteomic identification of differentially expressed proteins in mature and germinated maize pollen. Acta Physiol. Plant. 2011, 33, 1467-1474.

294. Zhu, Z.; Xu, F.; Zhang, Y.; Cheng, Y. T.; Wiermer, M.; Li, X.; Zhang, Y. Arabidopsis resistance protein SNC1 activates immune responses through association with a transcriptional corepressor. PNAS. 2010, 107, 13960-13965.

295. Zou, J.; Song, L.; Zhang, W.; Wang, Y.; Ruan, S.; Wu, W. Comparative proteomic analysis of Arabidopsis mature pollen and germinated pollen. J. Integr. Plant. Biol. 2009, 5, 438-455.

296. Zrenner, R.; Stitt, M.; Sonnewald, U.; Boldt, R. Pyrimidine and purine biosynthesis and degradation in plants. Annu. Rev. Plant Biol. 2006, 57, 805-836. 297. Zubarev, R. A. The challenge of the proteome dynamic range and its implications for in-depth proteomics. Proteomics. 2013, 13, 723-726. 